\title{
Null AND BEAM StEERING PERFORMANCE OF RECTANGULAR ARRAYS WITH DOLPH-CHEBYSHEV WEIGHTING
}

\author{
BY \\ VARAHRAM HEMMATI
}

A THESIS SUBMITTED TO THE FACULTY OF GRADUATE STUDIES AND RESEARCH IN PARTIAL FULFILLMENT OF THE REQUIREMENTS FOR THE DEGREE OF MASTERS OF APPLIED SCIENCE

OTTAWA-CARLETON INSTITUTE FOR ELECTRICAL AND COMPUTER ENGINEERING

\author{
DEPARTMENT OF ELECTRONICS \\ FACULTY OF ENGINEERING \\ CARLETON UNIVERSITY \\ OTTAWA, ONTARIO
}

(C) Varahram Hemmati, 2006 


$\begin{array}{ll}\begin{array}{l}\text { Library and } \\ \text { Archives Canada }\end{array} & \begin{array}{l}\text { Bibliothèque et } \\ \text { Archives Canada }\end{array} \\ \begin{array}{l}\text { Published Heritage } \\ \text { Branch }\end{array} & \begin{array}{l}\text { Direction du } \\ \text { Patrimoine de l'édition }\end{array} \\ \begin{array}{l}\text { 395 Wellington Street } \\ \text { Ottawa ON K1A ON4 }\end{array} & \begin{array}{l}\text { 395, rue Wellington } \\ \text { Ottawa ON K1A ON4 } \\ \text { Canada }\end{array}\end{array}$

Your file Votre référence ISBN: 978-0-494-26991-6 Our file Notre référence ISBN: 978-0-494-26991-6

NOTICE:

The author has granted a nonexclusive license allowing Library and Archives Canada to reproduce, publish, archive, preserve, conserve, communicate to the public by telecommunication or on the Internet, loan, distribute and sell theses worldwide, for commercial or noncommercial purposes, in microform, paper, electronic and/or any other formats.

The author retains copyright ownership and moral rights in this thesis. Neither the thesis nor substantial extracts from it may be printed or otherwise reproduced without the author's permission.
AVIS:

L'auteur a accordé une licence non exclusive permettant à la Bibliothèque et Archives Canada de reproduire, publier, archiver, sauvegarder, conserver, transmettre au public par télécommunication ou par l'Internet, prêter, distribuer et vendre des thèses partout dans le monde, à des fins commerciales ou autres, sur support microforme, papier, électronique et/ou autres formats.

L'auteur conserve la propriété du droit d'auteur et des droits moraux qui protège cette thèse. $\mathrm{Ni}$ la thèse ni des extraits substantiels de celle-ci ne doivent être imprimés ou autrement reproduits sans son autorisation.
In compliance with the Canadian

Privacy Act some supporting forms may have been removed from this thesis.

While these forms may be included in the document page count, their removal does not represent any loss of content from the thesis.
Conformément à la loi canadienne sur la protection de la vie privée, quelques formulaires secondaires ont été enlevés de cette thèse.

Bien que ces formulaires aient inclus dans la pagination, il n'y aura aucun contenu manquant.

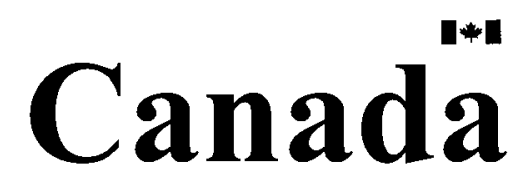




\begin{abstract}
This masters thesis describes the beamforming performance of rectangular arrays with Dolph-Chebyshev weighting. This type of weighting provides optimum mainlobe beamwidth for a fixed sidelobe level. However, the beamwidth of the mainlobe increases when a lower level is selected for the sidelobes. The null/beam steering performance of such an array, which can be used for GPS anti-jamming applications, is discussed and the results are presented for linear and rectangular arrays with 4 and 8 elements. The thesis also presents the optimum Minimum Variance Distortionless Response beamformer's performance in presence of interference.
\end{abstract}




\section{Acknowledgements}

I am very grateful for the opportunity to study under the supervision of Professor

Jim S. Wight and I would like to thank him for all the support and encouragement throughout my course of study as a Masters student at Carleton University. I would also like to thank my good friend and colleague Mr. Ehsan Gharibdoust for his help and support during my study and for his true friendship in life. I am thankful to Dr. Mike Vinnins, Dr. Jeff Bird, and Dr. Michel Clenet of the Defense Research \& Development Canada for their support and guidance toward this thesis.

Last but not least, I would like to thank all my friends and family, specially my parents Bahram and Mahsheed Hemmati for their love and support throughout my life and for dedicating their lives to help me realize my goals. 


\section{Table of Contents}

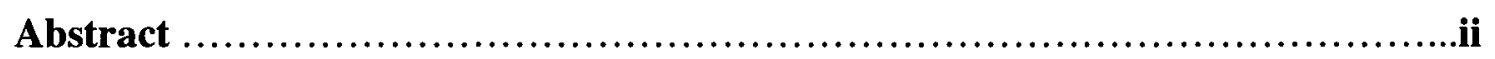

Acknowledgements ............................................................ ii

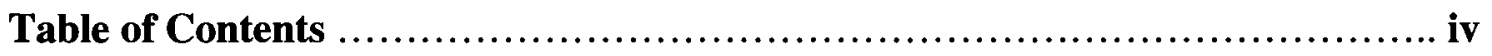

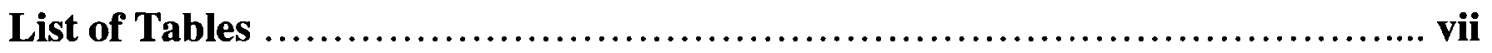

List of Figures .......................................................... viii

Notations and Abbreviations ............................................... xii

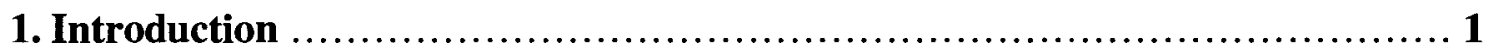

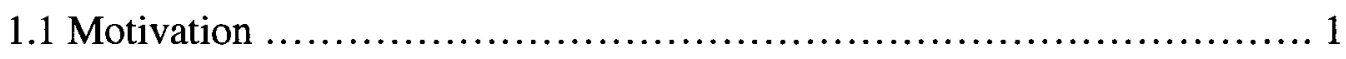

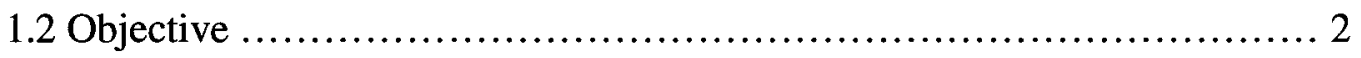

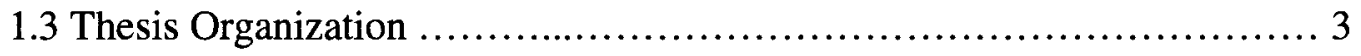

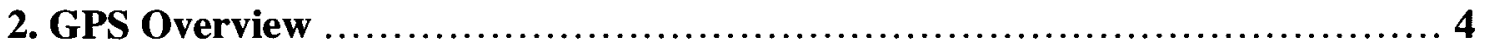

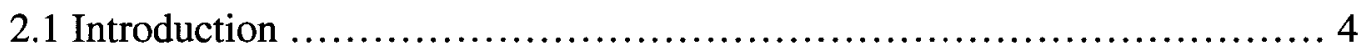

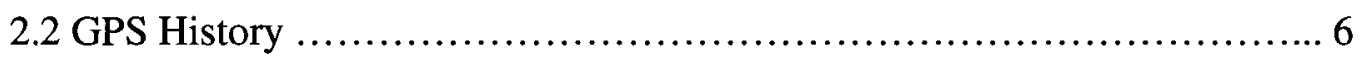

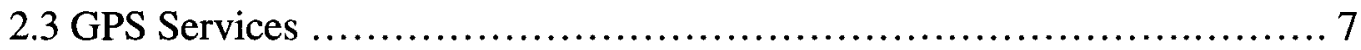

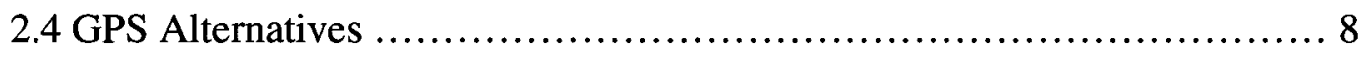

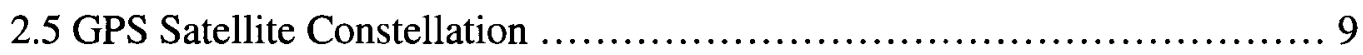

2.6 Signal Specifications .......................................... 10

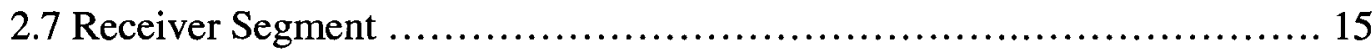

3. Interference and Anti-Jamming Overview .............................. 19

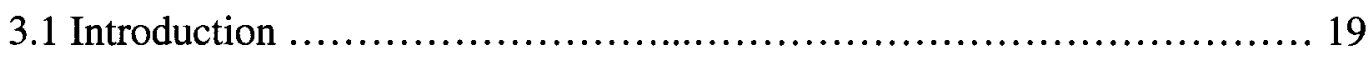

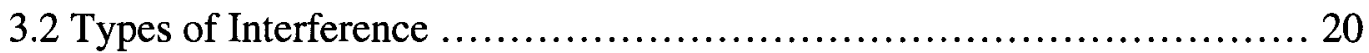




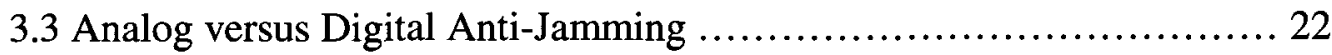

3.4 Anti-Jamming Techniques Overview ............................ 24

3.5 Anti-Jamming in Antenna Electronic Segment .................... 27

3.5.1 Multi-Element FRPAs .................................. 28

3.5.2 Multi-Element CRPAs .................................. 31

3.5.3 Null-Steering CRPAs .................................. 35

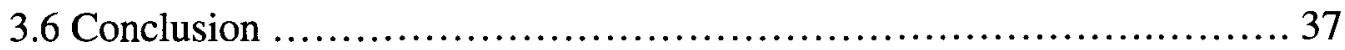

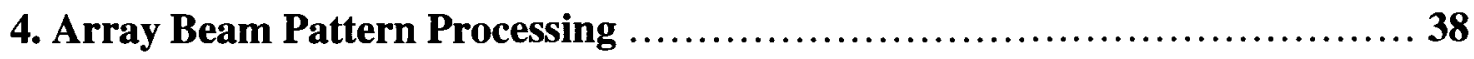

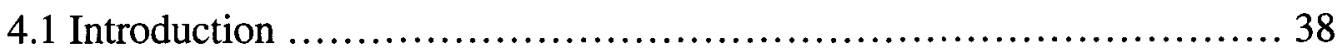

4.2 Linear Array Beam Pattern ..................................... 39

4.2.1 Linear Array Overview ..................................... 39

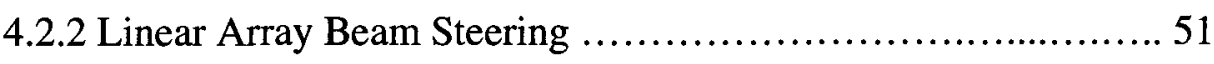

4.2.3 Dolph-Chebyshev Weighting for Linear Arrays ............... 53

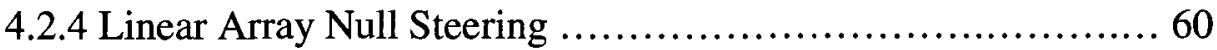

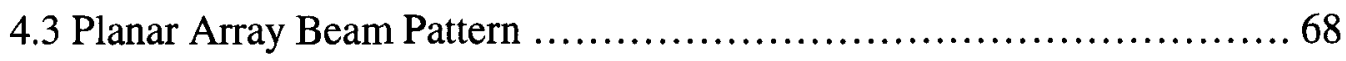

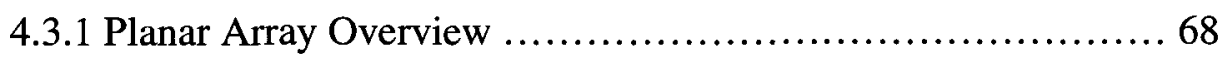

4.3.2 Dolph-Chebyshev Weighting for SURAs .................. 71

4.3.3 Null Steering Using Planar Arrays ........................... 74

4.3.4 Beam Steering Using Planar Arrays ........................ 79

4.3.5 Beam+Null Steering Using Planar Arrays $\ldots \ldots \ldots \ldots \ldots \ldots \ldots \ldots 81$

4.4 Minimum Variance Distortionless Response Beamformer ............. 82

4.4.1 MVDR Beamformer Overview ............................ 82

4.4.2 MVDR Performance in Presence of Interference ............... 85 


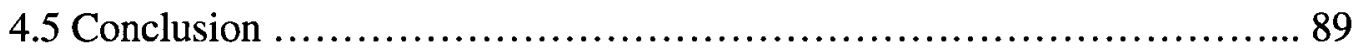

5. Concluding Remarks ................................................... 90

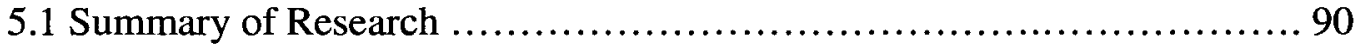

5.2 Possible Future Works ................................................ 91

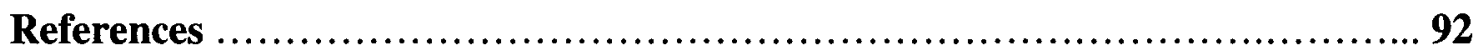

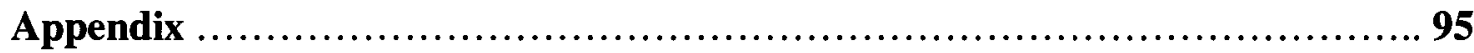




\section{List of Tables}

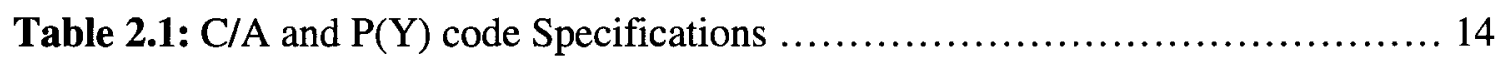

Table 3.1: Typical Interference/Jamming Types and sources $\ldots \ldots \ldots \ldots \ldots \ldots \ldots \ldots \ldots \ldots \ldots$

Table 3.2: Multi-Element Techniques Summary ............................. 34

Table 4.1: Time-Domain and Space-Domain Dualities ........................ 47

Table 4.2: Dolph-Chebyshev Weighting for a $4 \times 4$ SRA $\ldots \ldots \ldots \ldots \ldots \ldots \ldots \ldots \ldots \ldots \ldots$ 


\section{List of Figures}

Figure 2.1: Illustration of GPS Satellite Constellation ........................... 3

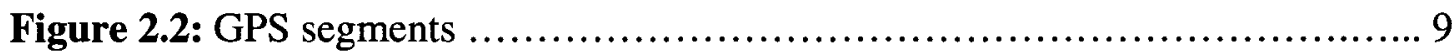

Figure 2.3: C/A Portion of Transmitted Signal on L1 ........................ 12

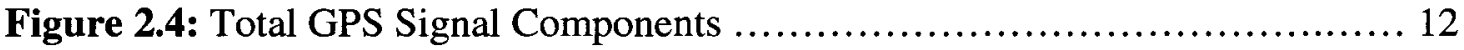

Figure 2.5: Illustration of Transmission and Reception of L1 Signal .............. 13

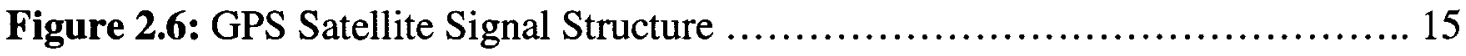

Figure 2.7: GPS Receiver Components ................................... 17

Figure 3.1: Illustration of Angular Coverage and Additional Anti-Jam .............. 25

Figure 3.2: Overview of GPS Anti-Jamming Techniques ...................... 26

Figure 3.3: Overview of Anti-Jamming Techniques in Antenna

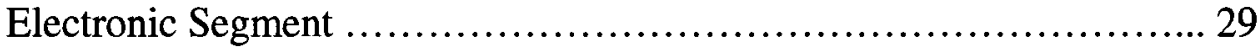

Figure 3.4: Basic Illustration of Multi-Element Cancellers ...................... 30

Figure 3.5: Multi-Element Canceller Application ............................................... 31

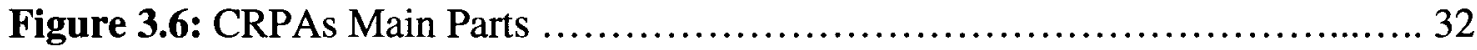

Figure 3.7: Null-Steering Performance versus FRPAs ........................... 36

Figure 4.1: Spherical Coordinate System ................................... 39

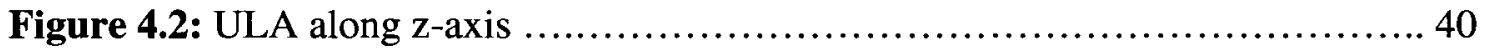

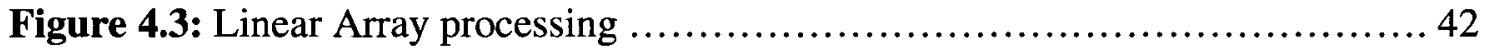

Figure 4.4: The Conventional Beamformer Processor ........................ 43

Figure 4.5: Angular Radiation Pattern of 4 and 8-element Array ................. 48

Figure 4.6: Array Factor for 4 and 8 -element Array ............................ 48

viii 
Figure 4.7: Effect of $\lambda / 4$ Element Spacing in $u$-space and $\theta$-space 49

Figure 4.8: Effect of $\lambda$ Element Spacing in $u$-space and $\theta$-space 50

Figure 4.9: Effect of $\frac{3 \lambda}{2}$ Element Spacing in $u$-space and $\theta$-space 50

Figure 4.10: Steering Beam to $60^{\circ}$ and $30^{\circ}$ in $u$-space with a 4-element Array......... 52

Figure 4.11: Steering Beam to $60^{\circ}$ and $30^{\circ}$ in $\theta$-space with a 4-element Array …....53

Figure 4.12: Steering Beam to $60^{\circ}$ and $30^{\circ}$ in $\theta$-space with an 8-element Array ......53

Figure 4.13: Beam Pattern in $\theta$-space using Dolph-Chebyshev Weighting .......... 57

Figure 4.14: Beam Pattern in $u$-space using Dolph-Chebyshev Weighting .......... 58

Figure 4.15: 8-element Array Pattern in $\theta$-space using Dolph-Chebyshev

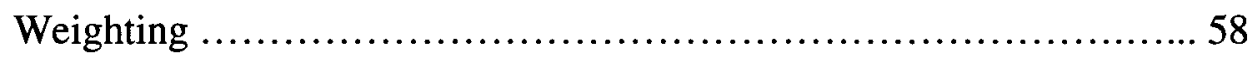

Figure 4.16: 8-element Array Pattern in $u$-space using Dolph-Chebyshev

Weighting 59

Figure 4.17: Dolph-Chebyshev Beam Pattern for sidelobe $=\left[\begin{array}{lll}-10 d B & -20 d B & -30 d B\end{array}\right]$ 59

Figure 4.18: Array Beam Pattern without Imposing Null ........................... 62

Figure 4.19: Array Beam Pattern with Zero-Order Null at $u=0.866$ or $\theta=30^{\circ} \ldots \ldots .63$

Figure 4.20: Array Beam Pattern with First-Order Null at $u=0.866$ or $\theta=30^{\circ} \ldots \ldots .63$

Figure 4.21: Array Beam Pattern with the Second-Order Null at $u=0.866$ or $\theta=30^{\circ}$ 64

Figure 4.22: 4-Element Array Beam Pattern with the Zero-Order Null at $u=0.866$ or $\theta=30^{\circ}$ 64

Figure 4.23: 4-Element Array Beam Pattern with the First-Order Null at $u=0.866$ or $\theta=30^{\circ}$ 65 
Figure 4.24: 4-Element Array Beam Pattern with the Second-Order Null

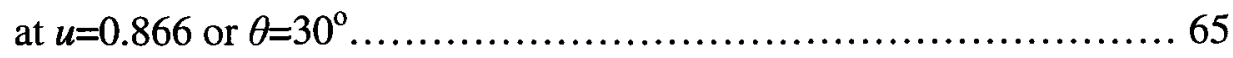

Figure 4.25: 8-Element Array with 3 Zero-Order Null

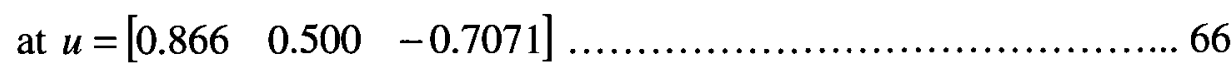

Figure 4.26: 8-Element Array with 3 Zero-Order Null

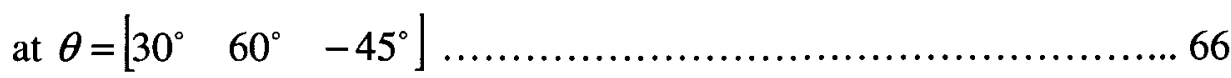

Figure 4.27: 4-Element Array with 3 Zero-Order Null

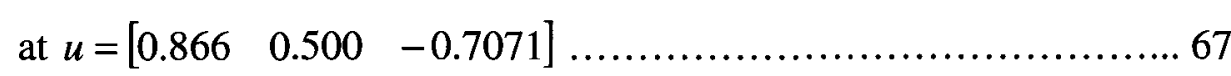

Figure 4.28: 8-Element Array with 3 Zero-Order Null

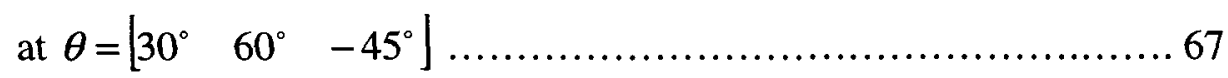

Figure 4.29: Rectangular Array Geometry ................................... 68

Figure 4.30: Two Views of 4x4 Standard Rectangular Array's Beam Pattern with Uniform Weighting ...................................... 70

Figure 4.31: Two Views of $8 \times 4$ Rectangular Array's Beam Pattern with Uniform Weighting 70

Figure 4.32: Beam Pattern of a $4 \times 4$ Standard Rectangular Array with Dolph-Chebyshev Weighting 72

Figure 4.33: Beam Pattern of a $8 \times 8$ Standard Rectangular Array with Dolph-Chebyshev Weighting 73

Figure 4.34: Different Views of $4 \times 4$ Array's Beam Pattern with null at $\theta=70^{\circ}, \phi=40^{\circ}$ or $u_{x}=0.72, u_{y}=0.60$ 76 
Figure 4.35: Different Views of $4 \times 4$ Array's Beam Pattern

with null at $\theta=5^{\circ}, \phi=40^{\circ}$ or $u_{x}=0.07, u_{y}=0.06$

Figure 4.36: Different Views of $8 \times 8$ Array's Beam Pattern

with null at $\theta=70^{\circ}, \phi=40^{\circ}$ or $u_{x}=0.72, u_{y}=0.60$

Figure 4.37: Different Views of $8 \times 8$ Array's Beam Pattern

with null at $\theta=5^{\circ}, \phi=40^{\circ}$ or $u_{x}=0.07, u_{y}=0.06$

Figure 4.38: $4 \times 4$ Array Optimum Beam Pattern with Three Nulls ................. 78

Figure 4.39: $8 \times 8$ Array Optimum Beam Pattern with Three Nulls ................ 79

Figure 4.40: Beam Steered to $u_{x}=0.26$ and $u_{y}=0.22$ Using $4 \times 4$ Array ........... 80

Figure 4.41: Beam Steered to $u_{x}=0.26$ and $u_{y}=0.22$ Using $8 \times 8$ Array $\ldots \ldots \ldots . . .80$

Figure 4.42: $4 \times 4$ Array with Mainlobe Steered to $u_{x}=0.26, u_{y}=0.22$, and Null Steered to $u_{x}=0.85, u_{y}=-0.49$

Figure 4.43: $8 \times 8$ Array with Mainlobe Steered to $u_{x}=0.26, u_{y}=0.22$, and Null Steered to $u_{x}=0.85, u_{y}=-0.49$

Figure 4.44: MVDR Beamformer 84

Figure 4.45: MVDR Beamformer in Presence of Interference 87

Figure 4.46: 4-Element Array Pattern in Presence of Interference 87

Figure 4.47: 8-Element Array Pattern in Presence of Interference 88

Figure 4.48: $4 \times 4$ and $8 \times 8$ Array Beam Pattern in Presence of Interference 88 


\section{Notations and Abbreviations}

\begin{tabular}{|c|c|}
\hline AoA & Angle of Arrival \\
\hline DOT & Department of Transportation \\
\hline GPSR & GPS Receiver \\
\hline UTC & Universal Time Coordination \\
\hline SPS & Standard Positioning Service \\
\hline PPS & Precise Position System \\
\hline SA & Selective Availability \\
\hline WAAS & Wide Area Augmentation System \\
\hline DGPS & Differential GPS \\
\hline GLONASS & Global Navigation Satellite System \\
\hline ESA & European Space Agency \\
\hline SoL & Safety of Life \\
\hline PRN & Pseudo-Random Noise \\
\hline RHCP & Right-Handed Circularly Polarized \\
\hline UWB & Ultra Wide Band \\
\hline $\mathrm{CW}$ & Continuous Wave \\
\hline AGC & Automatic Gain Control \\
\hline FRPA & Fixed Radiation Pattern Antenna \\
\hline CRPA & Controlled Radiation Pattern Antenna \\
\hline $\mathrm{W}_{\mathrm{i}}$ & Complex Weights \\
\hline$\phi$ & Angle in Azimuth Plane \\
\hline
\end{tabular}


$\mathrm{p}_{\mathrm{zn}}$

AMV

$\vec{u}$

$\vec{k}$

$\vec{w}^{H}$

$Y\left(\omega, k_{z}\right) \quad$ Output of a Linear Array located along the $z$-axis

$Y_{\psi}(\psi)$

$B_{\psi}(\psi)$

$B_{\theta}(\theta)$

UWLA

$R_{d B}$

$T_{m}(x)$

$x_{p}$

SURA

MVDR

$\sigma_{\omega}^{2}$

$\Lambda(\omega)$

$S_{n}$

$\sigma_{\text {int }}^{2}$

Angle in Elevation Plane

Array's Element Positions

Array Manifold Vector $\vec{v}_{k}(\vec{k})$

Direction Cosine Vector

Wavenumber Vector

Complex Weight Vector

Array Output in $\psi$-space

Array Beam Pattern in $\psi$-space

Array Beam Pattern in $\theta$-space

Uniformly Weighted Linear Array

The Sidelobe Level Compare to Mainlobe Level

$m$-th Order Chebyshev Polynomial

Zeros of $T_{N-1}(x)$

Standard Uniform Rectangular Array

Minimum Variance Distortionless Response

Spectral Height of White Noise Component at Each Element

Spectrum of the Interfering Signal

Noise Spectrum at Each Element

Interference to White Noise Ratio (INR)

xiii 


\section{Chapter 1: Introduction}

This chapter contains the introductory remarks for the thesis, which reports the research work done during the course of the author's studies at the Department of Electronics, Carleton University, Ottawa, Canada. This introduction chapter also gives an overview of the other chapters included in this report.

\subsection{Motivation}

As the number of applications for Global Positioning System increases, there will be more concern regarding the vulnerability of the system in intentional and unintentional interference and jamming scenarios. Military applications have been involved with GPS and its security issues for the past decades. Today, the vast use of the system in the military, calls for special attention to the design of the GPS receivers. The level of a receiver's sophistication depends on the type of application and operational budget. It can be as simple as analog filtering of the received signal, to several complicated pre- and post-correlation processing in both the antenna electronics and the GPS core segment. These sophisticated processors are very costly and physically large, which limits their use in many scenarios. Therefore a cheap and versatile unit, with less accuracy compared to higher end units, will be desirable in many military applications. 


\subsection{Objective}

The objective of this thesis is to research and develop the null steering algorithms (canceling jamming/interference signal spatially) for a small planar array with the minimum number of elements, to minimize the cost and the size of unit. The jamming/interference environments are not considered to be very sophisticated and only one or two sources are considered throughout the thesis. The algorithms were developed in MATLAB so they can be expanded upon future work, to include Angle of Arrival (AoA) estimation. The algorithms do not need a knowledge of the signal direction but will be provided with the information regarding the jammer direction to be able to cancel it spatially.

\subsection{Thesis Organization}

This thesis reports the results of researching the field of array processing for the purpose of improving the security of antenna electronics for use in GPS systems. The report contains five chapters. Following is a brief overview of each chapter.

Chapter 2 serves as a summary of GPS technology, its history, and its services to both military and civilian users. It also provides a view to the GPS signal structure and specifications. Included in this chapter, is basic information about the hardware segments of the GPS system. 
In Chapter 3, the different types of interference involved in GPS technology are discussed in some details. The chapter also provides a brief overview of the anti-jamming technologies currently available and the advantages/disadvantages of each technology.

Chapter 4 can be considered as the main body of this thesis and presents the research done on the array beam pattern processes for both linear and planar arrays. It also includes the results obtained by simulating (in MATLAB) the array null-steering algorithms for jamming/interference cancellation.

Chapter 5 presents the summary of the research and the concluding remarks. It also provides a brief overview of the work done by collaborating with the author's coresearcher on designing microstrip antennas for GPS application. At the end there are suggestions on the direction of future work. 


\section{Chapter 2: Global Positioning System Overview}

\subsection{Introduction:}

The Global Positioning System or GPS is a mature satellite navigational system consisting of 29 active/reserve satellites, which orbit in circular orbits. GPS receivers can determine their location accurately using the precise timing radio signal transmitted by GPS satellites. The system is very robust and allows the users to take advantage of this free service at any time of the day, in any weather, and any place on the surface of earth. The GPS has become a popular tool, and in some cases vital, due to its number of applications, utility, and low cost.

Its transportation applications include precision and nonprecision approach of aircrafts, marine navigation and harbor approach, and personal navigation devices. It has also telecommunication applications like precise timing and synchronization, which are especially important in remote areas. All these applications are highly desirable for military purposes, and that is the reason why it was first developed for military applications.

This increase in the use of the system, especially in transportation and military, calls for great effort to prevent any jamming or spoofing of the system. RF interference is the dominant problem in GPS use, in large part because of the low power of the GPS signal at the surface of the earth $\left(10^{-16}\right.$ watt or $\left.-160 \mathrm{dBw}\right)$. The GPS disruption 
mechanism is divided into two broad categories: unintentional and intentional disruption. Solar and ionospheric effects, interference from other RF sources, and signal blockage are considered as unintentional disruption. Jamming, spoofing, and deliberate efforts to shut down GPS service, on the other hand, are considered as intentional disruption [1].

This chapter gives a brief history of GPS and alternative systems. It also provides an overview of the services that the system provides to both civilian and military users. The basic specification of both satellite and receiver segment of the system are mentioned along with the transmitted and received signal construction. 


\subsection{GPS History}

In the early 1960s, several government organizations in the U.S. including the National Aeronautics and Space Administration (NASA), the U.S. Military, and the Department of Transportation (DOT) were showing interests in developing a global system for position determination using satellite technology. The attributes of an optimum system were considered as having: global coverage, the ability for high dynamic platform operation, the highest accuracy possible, and continuous/all weather operation. Now GPS is fully operational, globally accessible, and meets the criteria set in the 1960 s mentioned above [2].

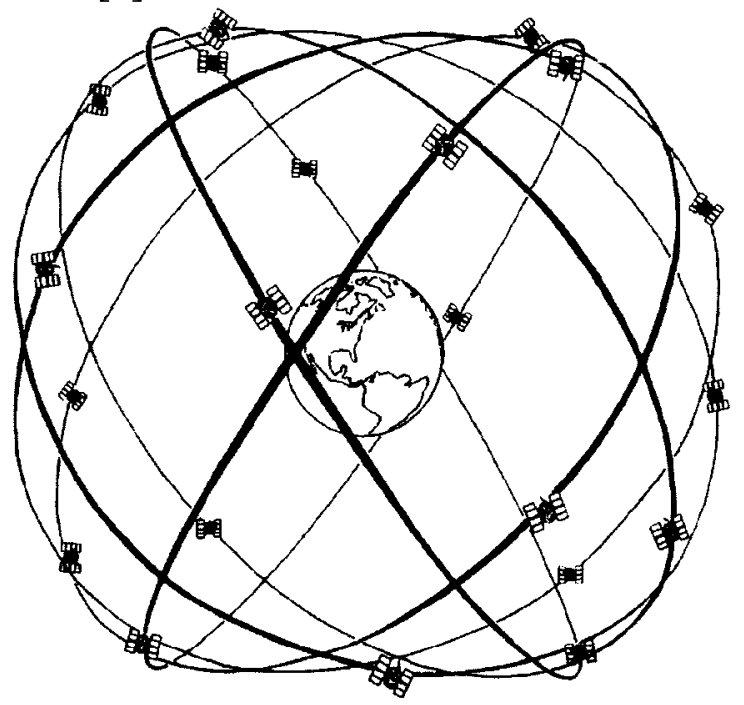

Figure 2.1: Illustration of GPS Satellite Constellation

Presently, any user with an appropriate GPS Receiver (GPSR) can enjoy the services that the system provides; continuous, accurate, worldwide, three-dimensional position and velocity measurements. Another important feature of the system is the dissemination of a form of Universal Time Coordination (UTC). 


\subsection{GPS Services}

GPS services are divided into two categories: the Standard Positioning Service (SPS) and the Precise Positioning Service (PPS). The SPS is designated for civilian use, whereas the PPS is restricted to authorized military applications and select government agency users. The SPS, which is available to all users worldwide without any restrictions, provides predictable accuracy of $100 \mathrm{~m}$ in the horizontal plane (after the U.S. government turned off the Selective Availability (SA) imposed on civilian users, the accuracy improved to about $10 \mathrm{~m}$ ) and $156 \mathrm{~m}$ in the vertical plane. At the time PPS provided a predictable accuracy of at least $22 \mathrm{~m}$ in the horizontal and $27.7 \mathrm{~m}$ in the vertical plane with a velocity measurement accuracy of $0.2 \mathrm{~m} / \mathrm{sec}$. Since August 2000, the GPS accuracy has been increased to within 2 meters for compatible receivers mostly used by military after introducing the Wide Area Augmentation System (WAAS). This system uses a series of ground stations for reference to calculate GPS correction messages, which are transmitted to GPS receivers using a series of additional satellites [3].

The GPS accuracy can be improved significantly to about $1 \mathrm{~cm}$ over short distances or 1-3 meters for normal GPS usage by using the Differential GPS (DGPS) technique. This technique uses a series of GPS receivers on the ground to calculate the precise difference between their actual known positions and the position provided by their received GPS signal. This difference is used to fix the signal for civilian GPS receivers for improved accuracy [4]. 


\subsection{GPS Alternatives}

Russia has its own radio navigation system called Global Navigation Satellite System or simply GLONASS, operated by Russia's Ministry of Defense. This system also provides the capability of worldwide three-dimensional position and velocity determination as well as time dissemination. GLONASS is very similar to GPS in many aspects. This system also consists of a 24 -satellite constellation in 3 orbital planes with 8 satellites per plane. It is also being monitored and controlled by a ground-monitoring network and provides service to various types of user receiving devices. Currently, there are several manufacturer of receiving equipments for this system within Russia as well as the rest of the world. Some of the new receivers can even take advantage of combined GPS/GLONASS signals. Both GPS and GLONASS have evolved from dedicated military systems to true dual-use [2].

The use of these navigation technologies is rapidly growing in numerous military and civil applications ranging from leisure hiking to spacecraft guidance. A new navigation system sponsored by the European Union and managed by the European

Space Agency (ESA) was introduced recently, with full development of operational satellite constellation by 2010 . It will consist of 30 satellites organized into three orbital planes, with a ground control segment very similar to GPS. 


\subsection{GPS Satellite Constellation}

The satellite constellation consists of at least 24 active satellites arranged in 6 nearly-circular orbital planes equally spaced and oriented at 55 degrees relative to the equator, with 4 satellites per plane orbiting at 26,560 kilometers above earth. This results in a sidereal period of 11 hours and 58 minutes. These satellites are spaced so that from any location on the earth surface at least 4 satellites will be visible above the horizon. Although these satellites are simple and unsophisticated compared to other modern satellites and spacecrafts, each of them carries a computer, dual atomic clocks, and several RF telecommunication devices. The on-board atomic clock helps the satellite keep its precise timing. The health and status of GPS satellites are monitored and controlled by a worldwide network of ground stations. The network also uploads navigation and other vital data to satellites several times a day. In addition to receiving these inputs, the on-board radio devices broadcast ranging codes and navigation data [2]. The three segments of the system and their inter-communication is illustrated in Fig. 2.2.

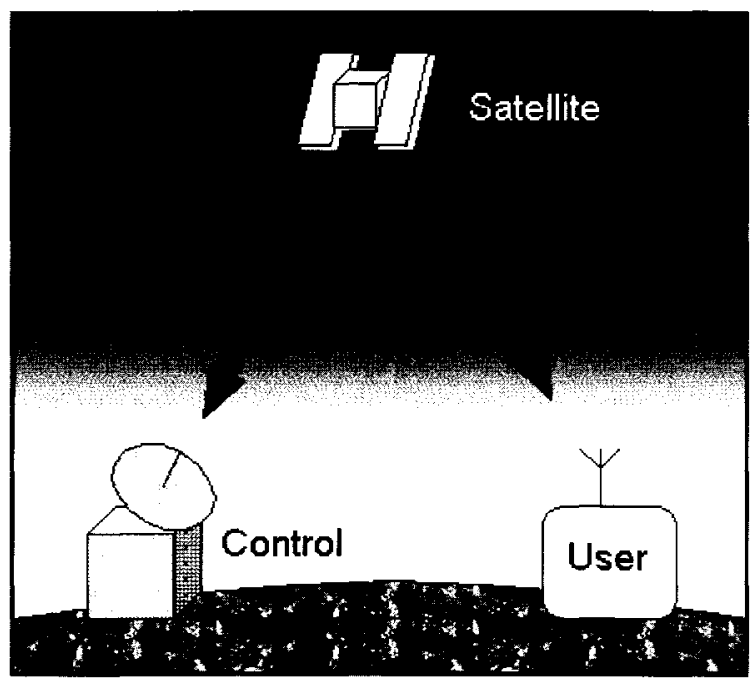

Figure 2.2: GPS segments [2] 


\subsection{Signal Specification}

Presently the satellites' radios broadcast the data on two frequency bands using a direct sequence Code Division Multiple Access (CDMA) technique. These two frequencies are called L1 (1575.42 MHz) and L2 (1227.60 MHz). A $50 \mathrm{~Hz}$ navigation message is sent on both of these bands [2]. Considering the fundamental frequency of the pure sine wave generated by the highly stable atomic clock on-board at $f_{0}=10.23 \mathrm{MHz}$, the carrier frequencies are set chosen as follow:

$$
\begin{aligned}
& f_{L 1}=f_{0} \times 154=1575.42 \mathrm{MHz} \\
& f_{L 2}=f_{0} \times 120=1227.60 \mathrm{MHz}
\end{aligned}
$$

A new frequency band called L5 (1176.45 MHz) has been proposed and is under study for use as a safety-of-life (SoL) signal for civilians. This new band falls in the range of the aeronautical navigation frequency, which is protected internationally. This will hopefully lead to little or no interference under all circumstances.

Each satellite uses a unique Pseudo-Random Noise (PRN) sequence (generally the satellites are identified by their PRN number, e.g. PRN 5 or PRN 12, also called ranging codes) to provide a distinguishable broadcast from other satellites in the constellation. PRN is a binary sequence with random properties similar to noise but it is generated by specific mathematical algorithms (or codes) and a tapped feedback shift register in hardware. It is then modulated on the GPS carrier waves using Binary-Shift Keying 
(BSK) modulation. This PRN code is mixed with the rest of the GPS data including a 50 bit per second navigation message, ephemeris (orbital) data, and clock-parameters [5], [6]. The total signal is then spread over a broad range of the spectrum and transmitted towards earth (Figure 2.3).

The received signal at the earth surface is very low in power (below the noise floor), which requires special techniques to distinguish the signal from noise. The PRN codes and GPS data should be separated in the receivers, this is done by using a locally generated PRN code (similar to the one generated by the satellite) and mixing it again with the received signal. This requires that the receiver generated code to be shifted in time until the two codes are exactly synchronous. The receiver then locks them and cancels them out, leaving the GPS data for further processing [5].

There are 32 different PRN codes available and distinguishable by the receivers, currently 31 codes are being used by 31 satellites. After starting up, the receiver looks for these codes and tries to lock with them; once one code locks then its satellite information can be decoded. The receiver will rapidly lock to the rest of visible satellites using the received data. The standard GPS Pseudo Random Noise (PRN) code is called the coarse acquisition (C/A) code, also known as the civilian code, and is only modulated on the L1 carrier at a chipping rate of $1.023 \mathrm{MHz}$ (each element in the PRN sequence is called a chip, and the number of chips per second is called the chipping rate). This is the code used by civilian receivers to acquire/decode the satellite signal on L1 frequency band and use it for L1 pseudo- range measurements. [2], [5] 


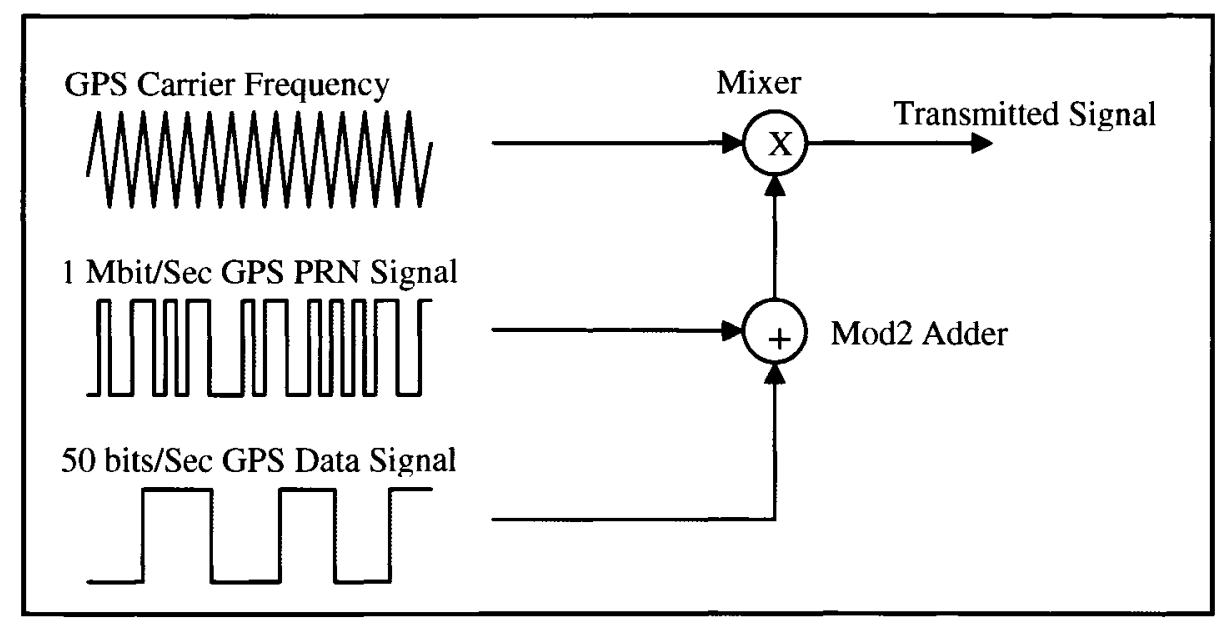

Figure 2.3: C/A Portion of Signal on L1 [5]

The precise or protected code, also known as $\mathrm{P}$-code (or $\mathrm{P}(\mathrm{Y})$ code), is a very long sequence of PRN binary biphase modulation on both L1 and L2 carriers at 10.23 MHz. This high bit rate of P-code allows the highest level of accuracy, thus the code is encrypted by the US department of Defense (under Anti Spoofing policy) and is only available to U.S. (and its allies) military and authorized users [2], [6]. The total transmitting signal components can be seen in Figure 2.4.

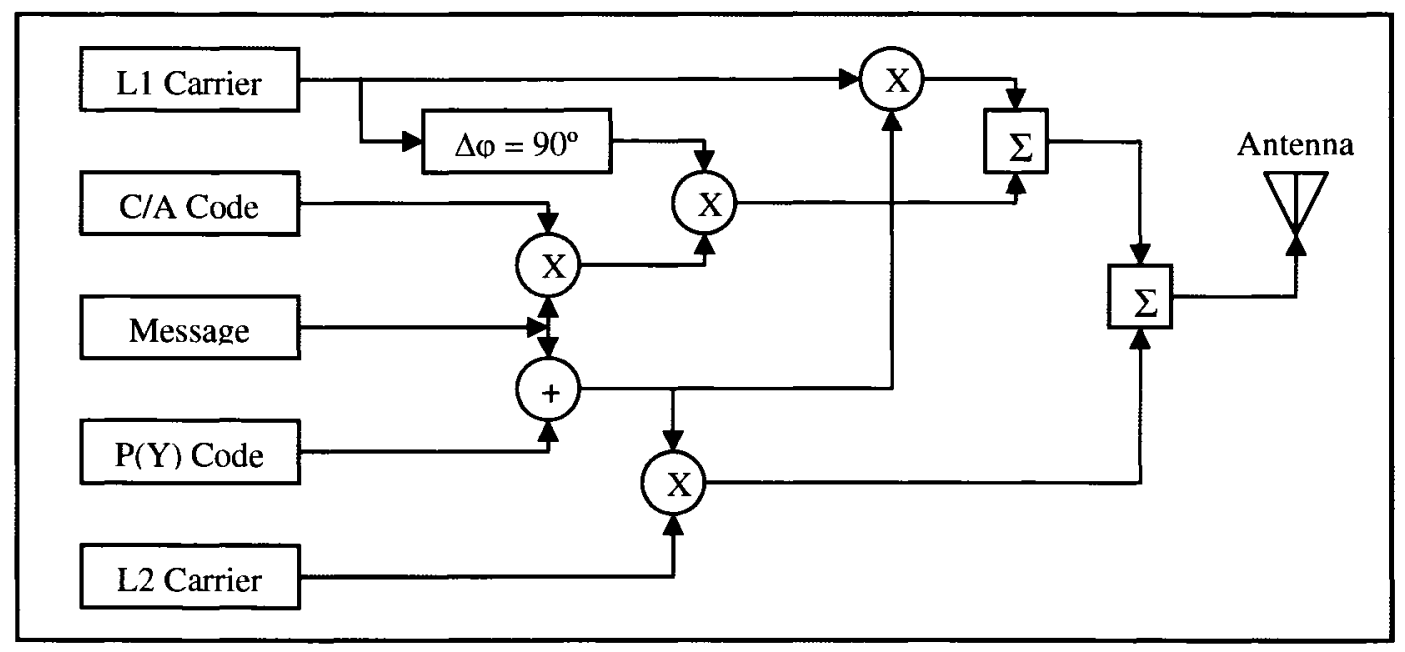

Figure 2.4: Total GPS Signal Components [2] 
Figure 2.5 shows an illustration of creating the L1 signal in the satellite segment and its reception at the GPS receiver end [6]. The satellite segment is shown on the left with the receiver segment on the right. The message data, which is mixed with the C/A carrier, is not shown below.
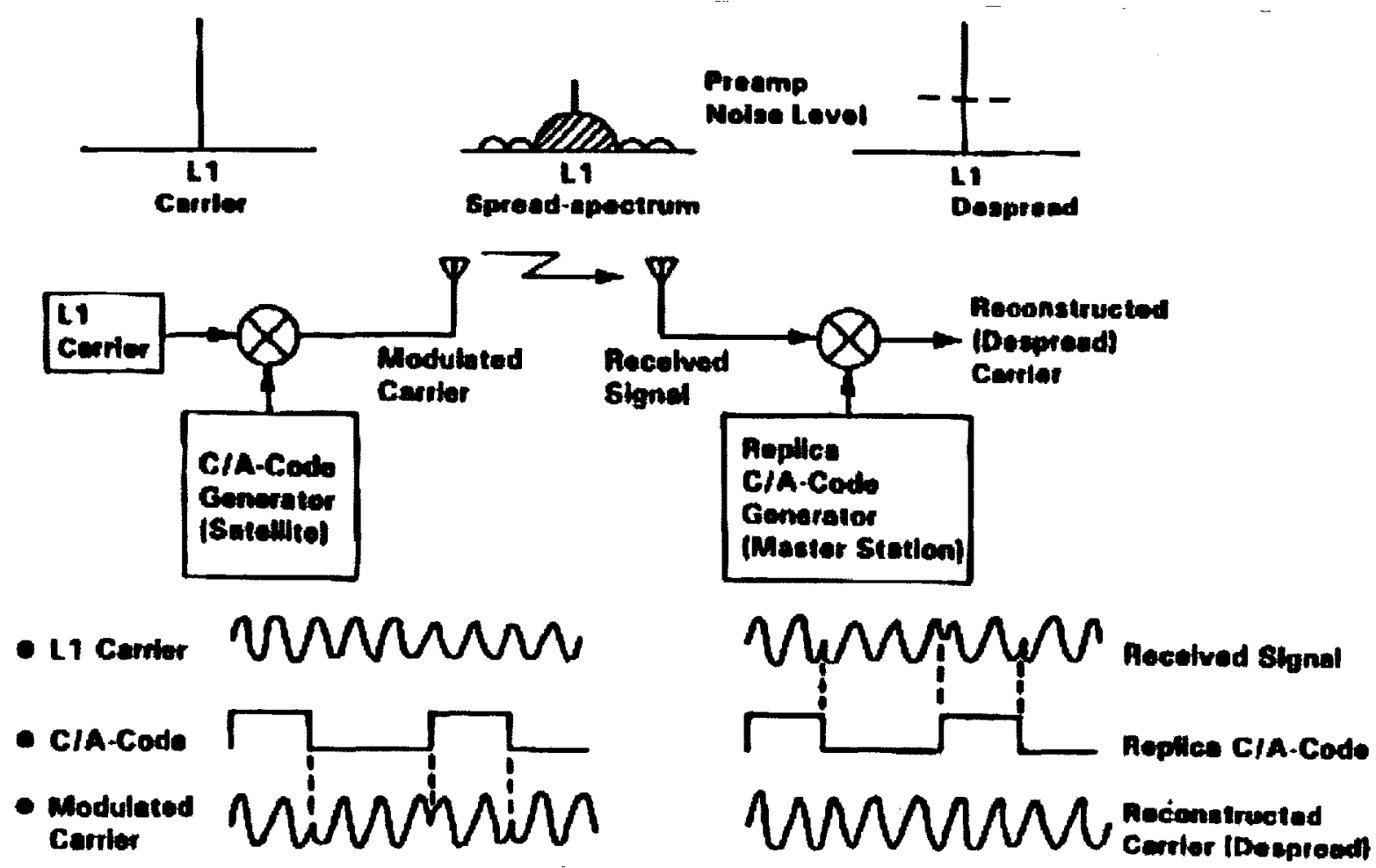

Figure 2.5: Illustration of Transmission and Reception of L1 Signal [6]

The P-code is very long compared to the C/A code (the P-code is a week long and the C/A code is $1 \mathrm{mSec}$ long), which helps making it difficult for unauthorized users to take advantage of the service. The code is reset at the beginning of each GPS week at midnight of Sat./Sun. The C/A code is also used for fast acquisition of the P-code. 
Table below gives the specification of $\mathrm{C} / \mathrm{A}$ and $\mathrm{P}(\mathrm{Y})$ code:

\begin{tabular}{|l|c|c|}
\hline Parameter & C/A code & P(Y) \\
\hline One Chip Range (meter) & 293.0 & 29.30 \\
\hline Code Repetition Interval & $1 \mathrm{mSec}$ & 1 week \\
\hline Chipping Rate (chips/sec) & $1.023 \mathrm{e} 6$ & $10.23 \mathrm{e} 6$ \\
\hline Chipping Period (nSec) & 977.5 & 97.75 \\
\hline
\end{tabular}

Table 2.1: C/A and $\mathrm{P}(\mathrm{Y})$ code Specifications

The signal breakdown of $\mathrm{L} 1$ and $\mathrm{L} 2$ is as follow:

Frequency $1=\mathrm{L} 1$ carrier $+\mathrm{P}(\mathrm{Y})$ code $+\mathrm{C} / \mathrm{A}$ code + Navigation Message

Frequency $2=\mathrm{L} 2$ carrier $+\mathrm{P}(\mathrm{Y})+$ Navigation Message

In other words:

- L1 Signal:

$S_{L 1}=A_{P_{L 1}} Y(t) N(t) \cos \left(\omega_{1} t\right)+A_{C / A}(C / A(t)) N(t) \sin \left(\omega_{1}\right)$

Where

$A_{P_{L 1}}=$ Amplitude of $\mathrm{P}(\mathrm{Y})$ code on L1 (about $-163 \mathrm{dBW}$ )

$Y(t)=$ Encrypted $\mathrm{P}(\mathrm{Y})$ code

$N(t)=50$ bit per second navigation message

$A_{C / A}=$ Amplitude of C/A code on L1 (about $-160 \mathrm{dBW}$ )

$C / A(t)=\mathrm{C} / \mathrm{A}$ code 
- L2 Signal:

$S_{L 2}=A_{P_{L 2}} Y(t) N(t) \cos \left(\omega_{2} t\right)$

Where

$A_{P_{L, 2}}=$ Amplitude of $\mathrm{P}(\mathrm{Y})$ code on L2 (about $-166 \mathrm{dBW}$ )

A complete illustration of GPS satellite signal can be seen below:

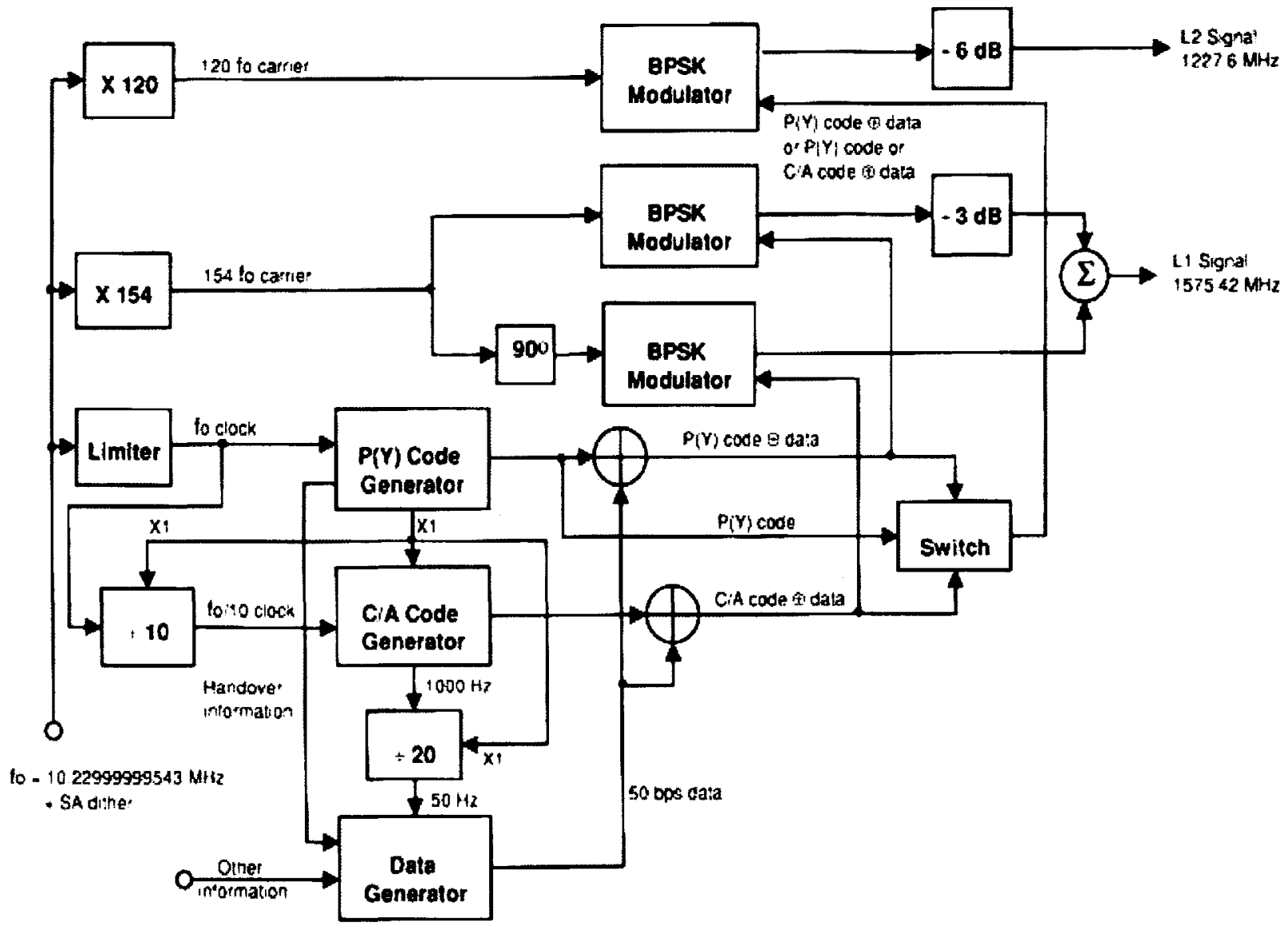

Figure 2.6: GPS Satellite Signal Structure [2] 


\subsection{Receiver Segment}

Since most of the GPS receivers operate passively (receive only), the system can provide service to an unlimited number of users. The accuracy of a GPS receiver depends in part on the number of radio receivers (channels) they have, since with more radio receivers the device can tune in and lock to more satellites (a dedicated signal channel for each satellite). Today, the cost of implementing GPS receivers has reduced significantly due to improvement in chip technology. Early receivers had up to eight channels for the L1 band, but the new generation of receivers (even the low cost consumer-level receivers) typically include twelve channels. High-end models can have 12 channels for the L2 band to correct for Ionospheric delays, since these delays are frequency-dependent and impact the L1 and L2 bands by a different amount (this difference is used to eliminate the entire Ionospheric delays) [6].

A typical GPS receiver (shown in Figure 2.7) consists of five main components: antenna, signal receiver, processor, input/output module, and power supply. The antenna part may include an array of several elements depending on the specification of the receiver and the required resistance to jamming. The antenna needs to be right-handed circularly polarized (RHCP), since the transmitted GPS signal from the satellites are RHCP. The typical coverage of GPS antennas is near hemispherical (about $160^{\circ}$ ).

The requirement for bandwidth of antennas depends on its ability to track the $\mathrm{P}(\mathrm{Y})$ code on both $\mathrm{L} 1$ and L2 bands, or just the C/A code on L1. In the first case, it is 
required to accommodate $20.46 \mathrm{MHz}$ bandwidth (two times the chipping rate of $\mathrm{P}(\mathrm{Y}$ ) code) for both frequency bands. If the receiver needs to track only the C/A code on L1, it needs to have only $2.046 \mathrm{MHz}$ bandwidth. There are different types of design suitable for GPS receivers including helical and microstrip (or patch) antenna. The latter is becoming more desirable due to its small size and profile, enabling the designer to reduce the antenna size in accordance with the rest of components. Low air resistance patch antennas are especially attractive for high dynamic aircrafts. There are several factors that need to be evaluated for the selection of an antenna such as antenna gain pattern, multipath performance, stability of the electrical phase center, and last but not least its ability to block as many interference sources as possible by having a deep null on low elevation [2], [5], [6].

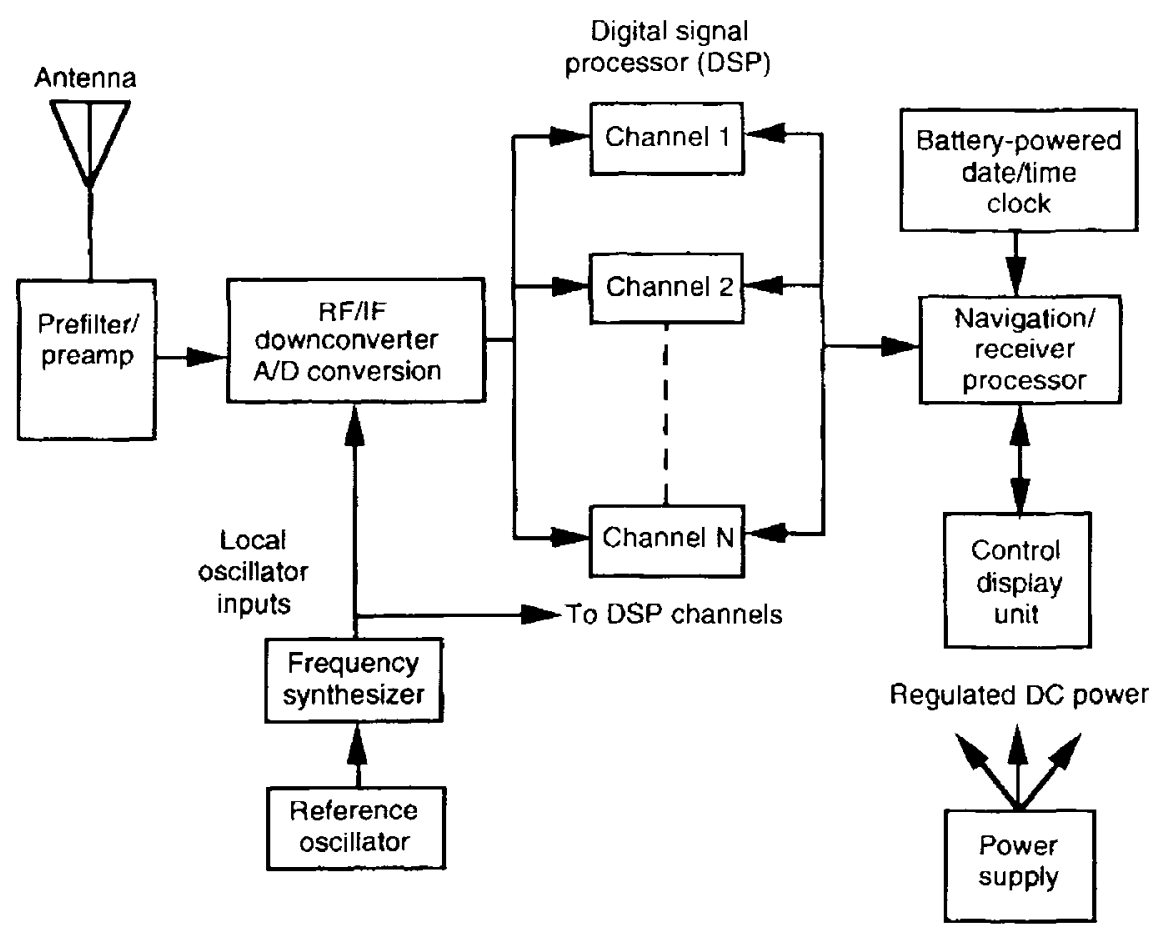

Figure 2.7: GPS Receiver Components [2] 
After the antenna, the received RF satellite signal passes through a passive bandpass prefilter to reduce the interferences that are out of the required bands. Usually a pre-amplifier block is placed after the filter to amplify the weak GPS satellite signal. The signal is then down-converted to an intermediate frequency (IF) and followed by analogto-digital converter, which samples and digitizes the IF signal. The minimum sampling rate of the $A / D$ converter is twice the bandwidth of the PRN codes (bandwidth for the $\mathrm{C} / \mathrm{A}$ code is slightly more that $2 \mathrm{MHz}$, and slightly more than $20 \mathrm{MHz}$ for the $\mathrm{P}(\mathrm{Y})$ code) to satisfy the Nyquist criterion, but it is normally chosen to be eight to twelve times the PRN codes' chipping rate. Oversampling at this stage reduces the receiver sensitivity to the $A / D$ converter quantization noise and therefore reduces the required number of bits in the converter [2].

The segment following the A/D converter is the DSP block, which can consist of a variety of sub-blocks depending on the receiver application and complexity. Normally, it includes between five to twelve parallel channels to simultaneously track the carrier and codes of several satellites. Each channel has its own code/carrier tracking loops and can perform code/carrier-phase measurements and navigation message data modulation. Then the measurement and demodulated navigation message are forwarded to the navigation processor [2], [6]. 


\section{Chapter 3: Interference and Anti-Jamming Overview}

\subsection{Introduction}

The use and availability of GPS has become a vital part of transportation infrastructure and has emerged as a crucial tool to an already large number of applications for military air, land, and maritime operations. This makes the GPS interference scenarios very important and as a result its vulnerability to different type of interferences (both unintentional and intentional) is being discussed and researched in military organizations around the world. The GPS receivers rely on RF signals and this makes them especially vulnerable to RF interferences, due to the very low power levels of GPS signals. These interferences can degrade the navigation accuracy and in extreme cases it can result in a complete loss of tracking in the receivers.

This chapter contains sections on types of interference, analog versus digital techniques, overview of anti-jamming techniques, and some details on techniques using multi-element antenna arrays. 


\subsection{Types of Interference}

As mentioned earlier, GPS interference mechanisms are divided into two categories: unintentional and intentional. Unintentional interferences from nature generally offer no insurmountable problem, but the unintentional interferences caused by human sources should be dealt with more carefully. The unintentional interferences include: Mobile satellite services, Ultra Wideband (UWB) devices, TV channels $(23,66$, and 67), personal electronic devices (PEDs), over-the-horizon radar, and military communication systems.

Intentional jamming is not only a real threat; it is also much more dangerous than the unintentional jamming. It denies the GPS signal to the user by concentrating jamming energy in the frequency bands allocated to GPS (simply called jamming). A more sophisticated intentional threat to the system is spoofing, which works on deception of the GPS navigation signal by attempting to capture the GPS receiver's tracking loops. It then creates believable but false signals (e.g. true lies) and over-powers the desired signals to trick the receivers, forcing them to output wrong navigation data. It is exceptionally dangerous to military operations, since it can misguide military hardware such as smart bombs. However, spoofing is much more complex to achieve than jamming and well-designed modern receivers are almost invincible against it. The reason is that for a successful attack on the receiver's navigation filter, the fake signal must be made completely believable and its operation must remain unobservable [7]. 
A common type of jamming is brute force jamming, which simply aims very powerful noise or continuous wave $(\mathrm{CW})$ signals at the receivers. This broadband signal is just random noise and it puts a "noise blanket" over the desired signal. These signals are intentionally located at, or very close to, the $\mathrm{L}$ frequency bands, and if they are well placed these signals can force the receiver to lose its lock to the satellites and their navigation data. Other jamming techniques can get extremely sophisticated and hard to detect. These smart techniques include: swept CW signals, hopping frequency signals, or tailored signal pulsing. Smart jammers typically use the same average power constraint; if pulsed noise and pulse CW signals are used then jammers can achieve a much higher peak power for the same average power. A very hard-to-detect jamming technique is frequency hopping, which mainly attacks the receiver's mixers and amplifiers [2]. The following table summarizes the typical interference and jamming types and sources.

\begin{tabular}{|l|l|}
\hline \multicolumn{1}{|c|}{ Interference/Jammer Type } & \multicolumn{1}{|c|}{ Interference/Jammer Source } \\
\hline Wideband-Gaussian & Intentional noise jammers \\
\hline Wideband phase/frequency modulation & $\begin{array}{l}\text { TV transmitters' harmonics, near-band } \\
\text { microwave link transmitter }\end{array}$ \\
\hline Wideband-spread spectrum & Intentional spread spectrum jammers \\
\hline Wide-band pulse & Radar transmitters \\
\hline Narrowband phase/frequency modulation & $\begin{array}{l}\text { AM stations transmitters' harmonics, CB } \\
\text { transmitters' harmonics }\end{array}$ \\
\hline Narrowband-swept continuous wave & $\begin{array}{l}\text { Intentional CW jammers, FM stations } \\
\text { transmitters' harmonics }\end{array}$ \\
\hline Narrowband-continuous wave & $\begin{array}{l}\text { Intentional CW jammers, near-band } \\
\text { unmodulated transmitters' carriers }\end{array}$ \\
\hline
\end{tabular}

Table 3.1: Typical Interference/Jamming Types and sources 
There are a number of factors that help reduce the effects of RF interference. Being in the line of sight of the GPS antenna (unobstructed) is the only way that RF interference can have full effect on the receiver. In aviation applications, the RF interference source is normally located at ground level while the GPS receiver antenna is elevated during flight. The altitude increases the line-of-sight (which creates the danger of having more interference sources in sight) but since the antennas are usually located on top, the body of aircraft will help to block the interference.

\subsection{Analog versus Digital Anti-Jamming}

Analog-based anti-jam techniques, based on the technology developed in the 70's, used to be the main response to the jamming scenario until recently. These techniques mainly include controlling the radiation/reception of the GPS receiver antenna using controlled radiation pattern algorithms, decreasing the effect of interference by nulling out the offending interference signal. The anti-jamming analog systems generally consist of several antenna elements and a set of analog phase shifters, which adaptively control and set the weights of antenna elements output. This results in these systems' large size, huge weight, and high cost, which limit their use in many situations. Another issue with analog systems is their accuracy and flexibility in controlling the phase of antennas. This is due to the naturally limited precision of analog phase shifters. There is also a problem with system performance in a high dynamic environment, since the antenna elements' weights need to be calculated using an iterative algorithm [8]. 
The problems and limitations associated with the analog anti-jamming techniques and recent developments in the field of digital signal processing has been the main reason for the extensive interest in use of digital technology in GPS anti-jamming. Using digital signal processing, the signal phase and amplitude can be precisely controlled during the mixing of the signal coming from individual elements of antenna array. This precise control gives the digital techniques the ability to calculate the desired weight and accurately applying them to the signals, which leads to both deeper nulls and increased steering gain for the system (beamsteering).

Another important advantage of digital anti-jamming (a result of precise phase control) over the analog techniques is the almost complete elimination of in-phase and quadrature-phase (I/Q) imbalance, which greatly improves the system's nulling performance. This digital performance is also significantly faster due to the fast rate of complex signal weights' adaptation. The fast adaptation is very important for responding to changes in spatial orientation of the jamming signal and also helps the digital antijamming process to respond to a dynamic jamming environment much faster compared to the analog system. A Hilbert filter, generating the I/Q signals necessary for the antijamming process, can be used to achieve any level of negative image rejection [2], [8].

In addition to advantages mentioned above, the digital systems allow the adaptive weights to be applied directly to the same signal from which they are calculated. This lead to deeper nulls and improved anti-jamming performance in an environment in which the direction of jamming signals is changing rapidly [8]. 


\subsection{Anti-Jamming Techniques Overview}

The GPS anti-jamming technologies can be divided into three main categories: antenna-based anti-jamming, adaptive filters, and receiver signal processing. Antenna based techniques can provide the users with acceptable protection against narrowband and wideband jammers. The down side to these techniques is their limitations to larger number of jammers. Another issue is the design and platform requirements of antenna spacing and sizing, which limit their use with many systems. Using adaptive filters, effective anti-jamming can be achieved against narrowband or CW jammers. But they, like antenna techniques, have limitations against a highly dynamic environment. On the other hand, signal processing techniques are very low cost to develop, but have limitations on their anti-jamming performance (about $20 \mathrm{~dB}$ anti-jamming improvement).

The anti-jamming performance of each technology can be analyzed using the following measures:

- The amount of additional anti-jamming that the technology provides compared to an unprotected receiver. This is usually equivalent to the depth of spatial nulls at the direction of jamming signal (the relative gain of the antenna in the direction of the GPS and jamming signal).

- The ability to cancel larger number of jammers, depending on whether they are narrowband or wideband, and their direction of arrival.

- The ability to receive GPS signals while spatially nulling a jamming signal (or simply the angular coverage of a signal in the presence of nulling). To estimate 
the range of the angles for successful reception of the GPS signal, it is normally assumed that the GPS receiver will lose track of the desired signal if either the antenna gain in the direction of GPS signal drops below -10 $\mathrm{dBi}(\mathrm{dBi}$ refers to the antenna gain in a given direction with respect to an isotropic radiator), or signalto-noise (SNR) degradation as the IF increases above $10 \mathrm{~dB}$ relative to the unjammed SNR with a $0 \mathrm{dBi}$ antenna gain [2], [9], [10].

These angular coverage and additional anti-jam measures are illustrated in the following Figure in presence of a single jamming source:

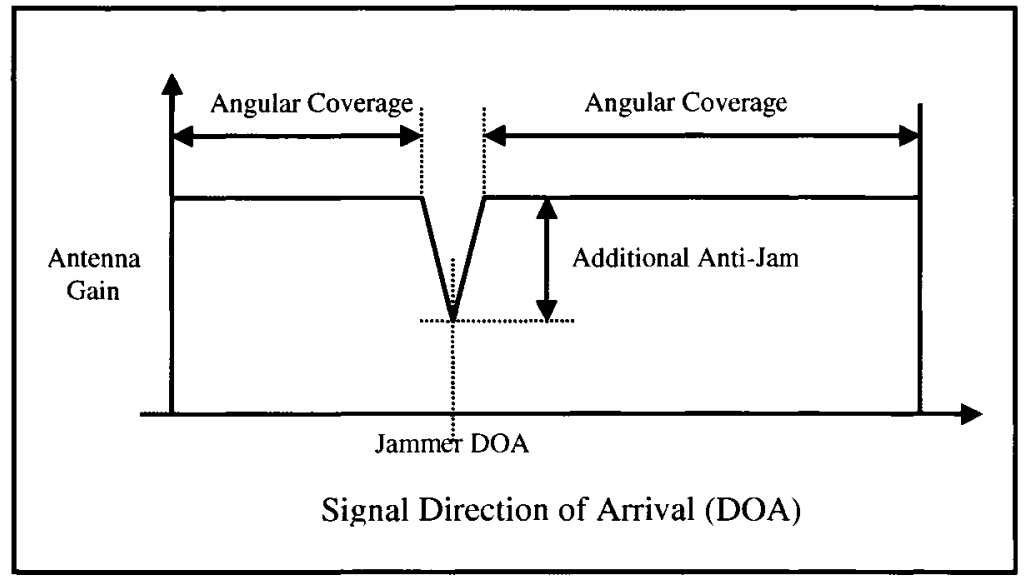

Figure 3.1: Illustration of Angular Coverage and Additional Anti-Jam

As it is shown in Figure 3.2, the anti-jamming techniques can be performed in three separate parts of a GPS receiver; the RF front end (also called antenna electronics), the DSP processor, and GPS core. The techniques done in the DSP processor prior to feeding the signal to the core is called pre-correlation and the techniques done in the core are called post-correlation. The post-processing techniques done in the GPS core module, 
aim at increasing the tracking threshold of the loops and allowing them to operate at lower SNR values [10].

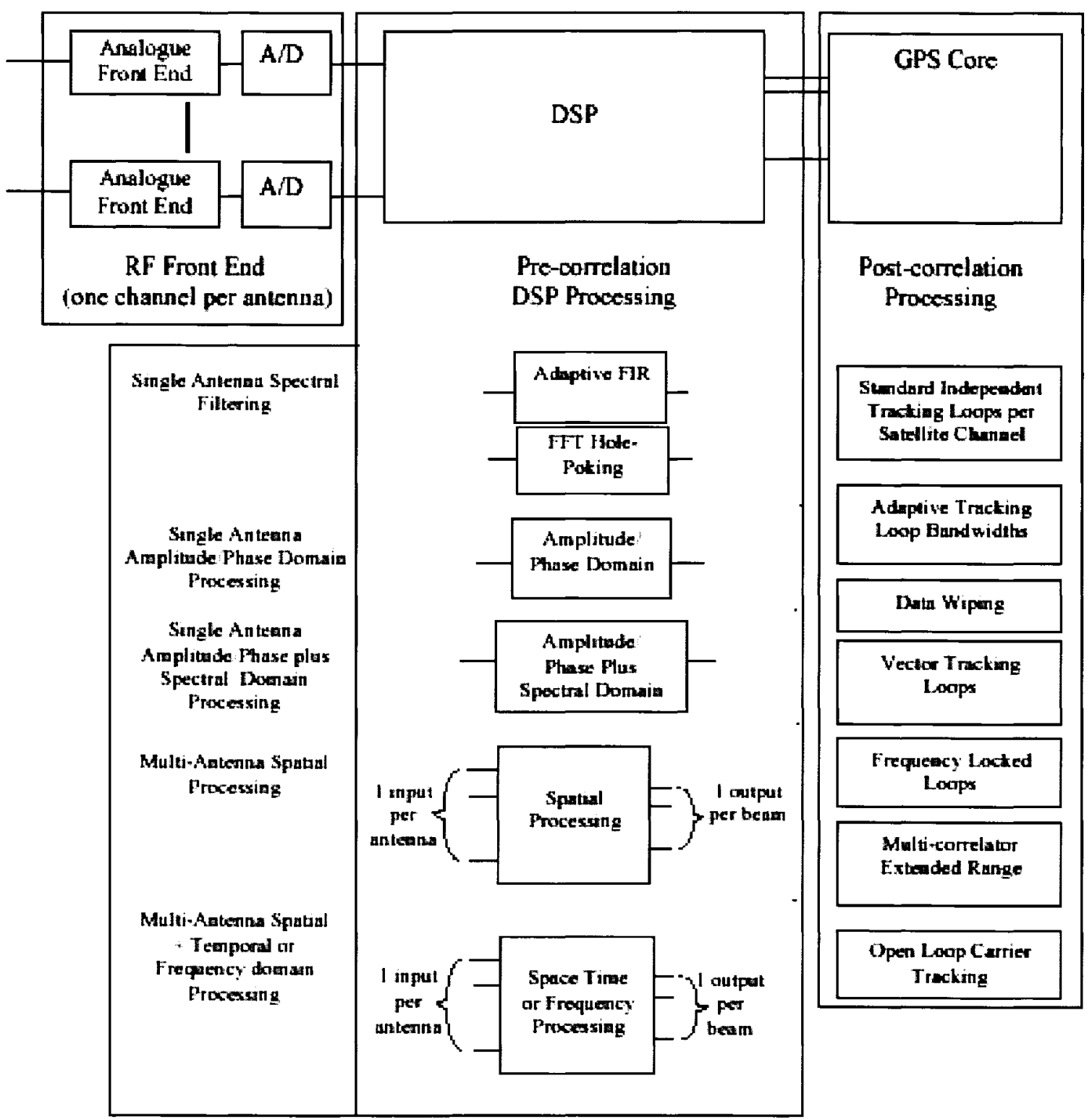

Figure 3.2: Overview of GPS Anti-Jamming Techniques [10] 


\subsection{Anti-Jamming in Antenna Electronics Segment}

Using several antenna elements makes an adaptive antenna array very effective against broadband jammers, since they cancel out the jammer signal in the spatial domain. However these adaptive antennas are generally very sensitive to direction of arrival (DOA) of the jammer and how close is its direction to the desired GPS signal. It should be mentioned that using multiple elements techniques obviously increase the cost and size of the systems incorporating these techniques [10].

There are also several analog anti-jamming techniques that can be done in the antenna electronics including: use of an analog front end with a high dynamic range, analog filtering, and use of an Automatic Gain Control (AGC) unit for power estimation and jammer detection. Use of low distortion mixers and amplifiers and high precision A/D converters helps achieve higher dynamic range. Having a front end amplifier with high dynamic range helps avoid harmonic distortions and saturation in amplifiers (which can significantly worsen the performance of other anti-jamming segments) caused by strong jammers.

Analog band-pass filtering, as mentioned earlier, can be considered to reject the out-of-band jammers. Band-pass filters with low insertion loss (avoiding excessive degradation of the GPS signal SNR), sometimes incorporated with the GPS antenna segment and placed prior to the amplifier; help prevent saturation of the amplifier by strong out-of-band jammers. AGCs can be used to detect the presence of a jammer by 
continuously monitoring the power level of the received signal. Since the GPS signal is below the thermal noise level, any increase in power level yields the presence of jammer(s) and then the strength of the jammer signal can be estimated. This unit also adjusts the gain of the RF front end to keep the signal power within the dynamic range of the A/D converters [9], [11], [12].

Many receivers incorporate Fixed Radiation Pattern Antennas (FRPA), which are normally designed to have deep nulls near the horizon where most of the jamming signals come from. Additionally Controlled Radiation Pattern Antennas (CRPA) can be used to achieve the null steering, which continuously adjust the channels' weights and the radiation pattern of the array to steer a null at the direction of jammer. Figure 3.3 shows an overview of anti-jamming techniques that can be incorporated in the antenna electronic segment. Due to their advantages, the emphasis in this thesis is on the techniques with multiple antenna elements [11].

\subsubsection{Multi-Element FRPAs}

As it can be seen in Figure 3.3, there are two techniques using multiple FRPAs: switched antenna and multi-element canceller [12]. The first technique considers the multiple FRPAs of the system, each with a different radiation pattern aimed at a different direction covering only a portion of the sky, and the system switches between them to find the optimum radiation pattern. There is not enough flexibility in the overall radiation pattern and the requirement of several FRPAs can be very costly. 


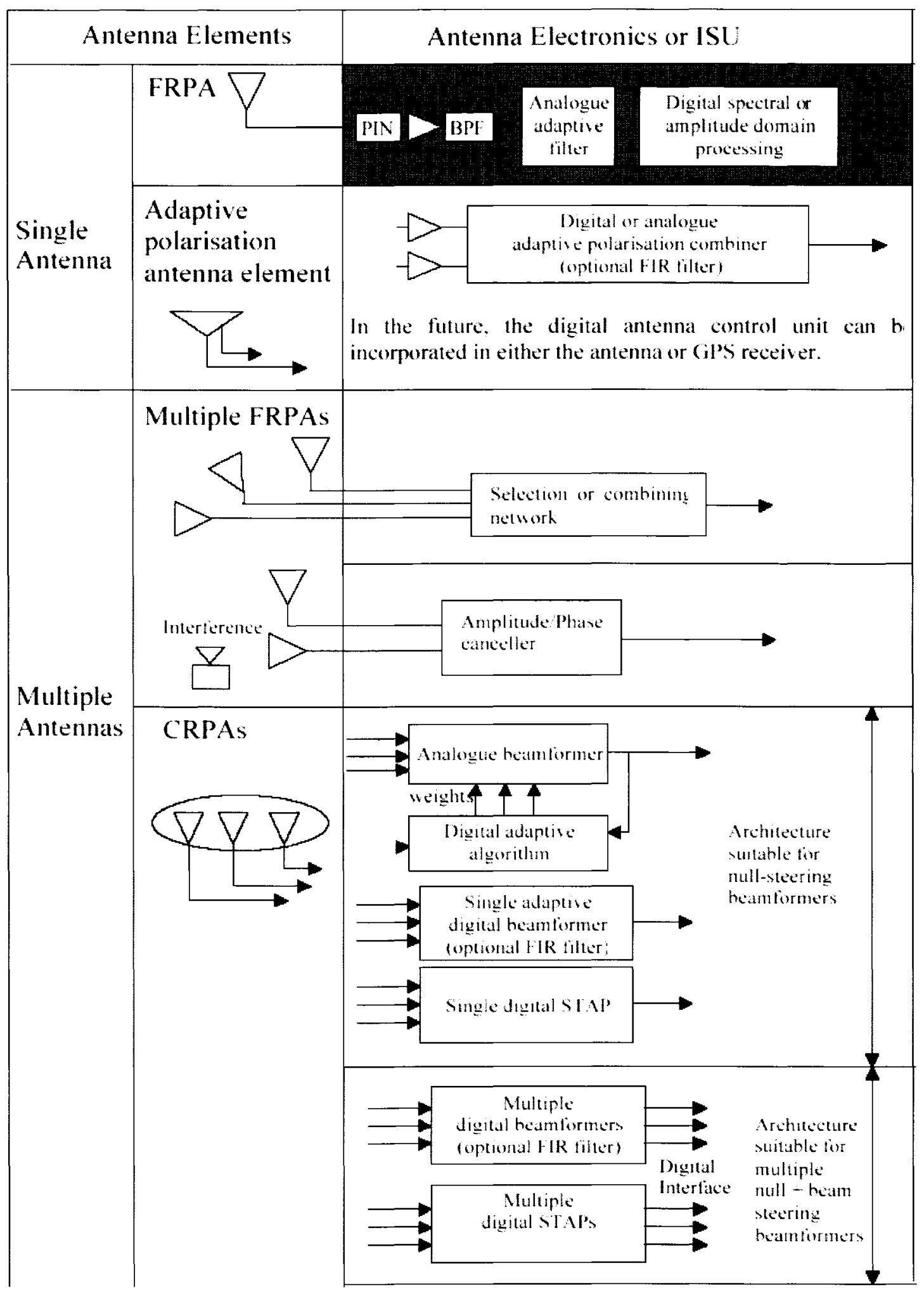

Figure 3.3: Overview of Anti-Jamming Techniques in Antenna Electronic Segment [12] 
Switched antenna techniques, because of their spatial diversity, can be useful for signal fading (reducing the effect of signal multi-path effects).

The multi-element canceller techniques use two FRPAs, one directed at the direction of the GPS signal (the primary FRPA) and the second FRPA directed at the jammer (the auxiliary FRPA). The primary one receives a mix of GPS plus jammer signal, and the auxiliary FRPA mostly picks up the jammer signal. Then these two received signals are combined to eliminate the jammer signal, requiring the calculation of correct phase and amplitude [11], [12]. This technique is shown in Figure 3.4.

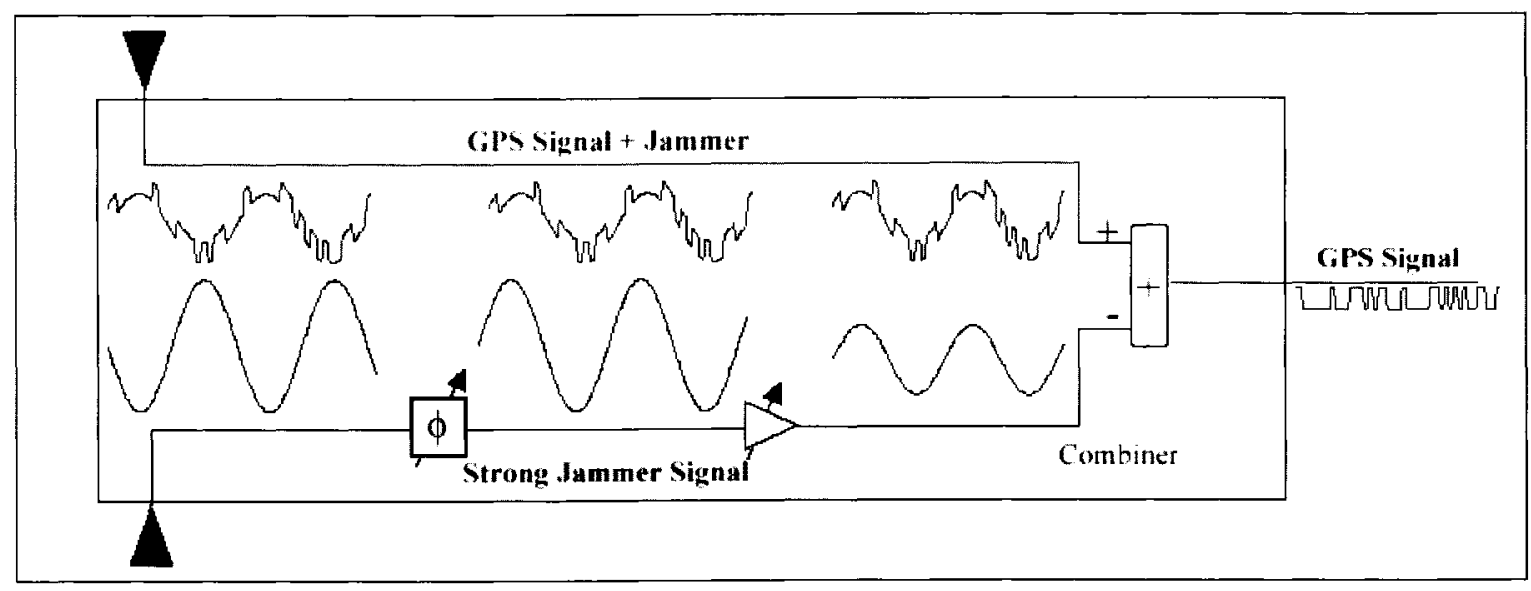

Figure 3.4: Basic Illustration of Multi-Element Cancellers [11]

The performance of this technique is acceptable only if the auxiliary FRPA does not pick up the GPS signal, otherwise it would also cancel the GPS signal. This makes the technique very sensitive to the altitude of the aircraft. This technique is mainly developed for aircraft application, since it can significantly attenuate the low elevation 
jammers. A primary and auxiliary FRPAs' installation is illustrated in Figure 3.5. The FRPAs should be installed accurately in a vertical orientation on the top and bottom of the aircraft, providing the best performance when the aircraft is flying horizontally.

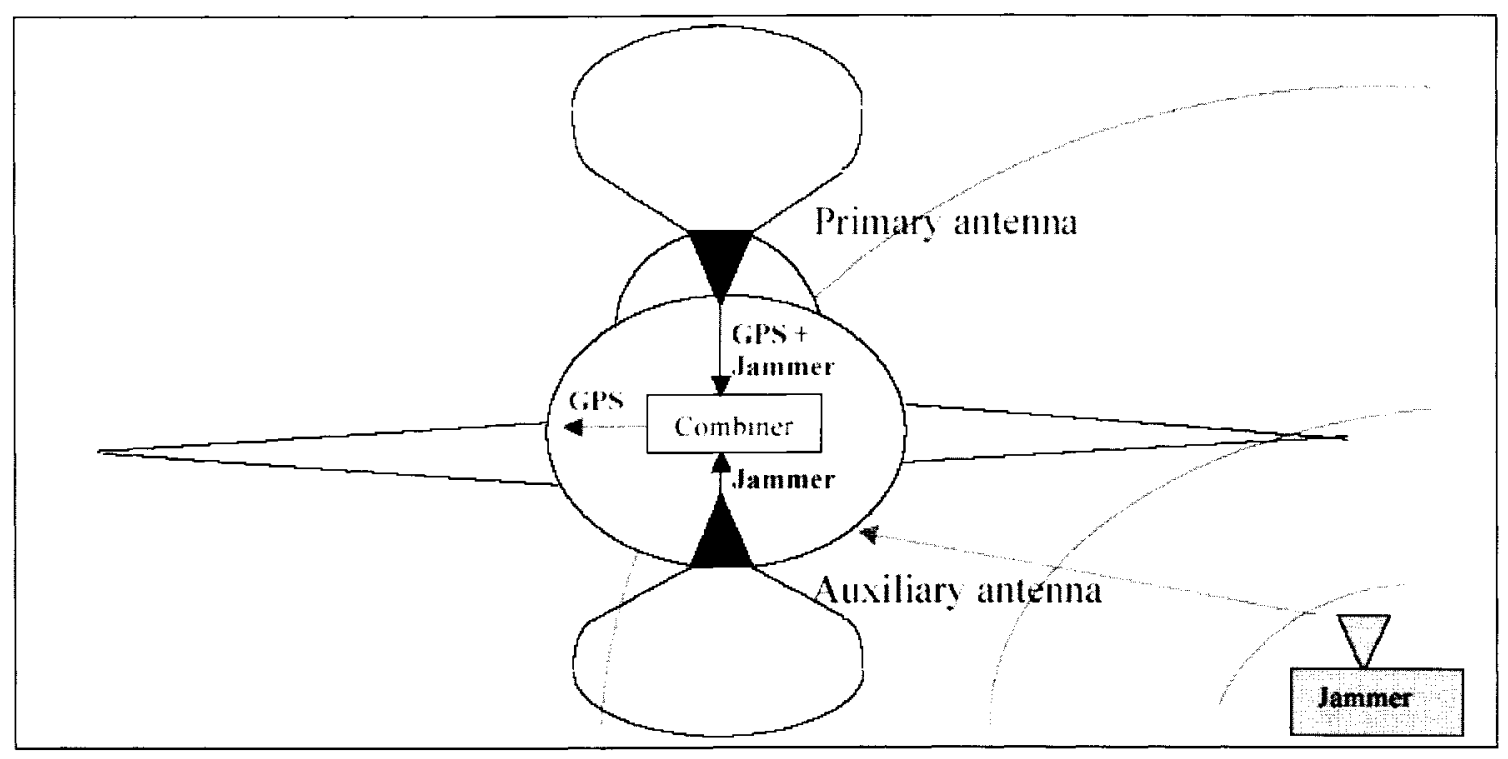

Figure 3.5: Multi-Element Canceller Application [11], [12]

\subsubsection{Multi-Element CRPA's}

CRPA's act like a single antenna element with an adjustable radiation pattern, and consist of two parts (shown in Figure 3.6): an antenna array with several elements (normally between 4-8 elements) and beamformer(s), which combines the signal picked up by each element and creates the adjustable radiation pattern. The simplest technique using CRPAs is null steering, which steers nulls at the direction of jammers and tries to keep constant gain in all other directions. Ideally it attenuates all type of jamming 
waveform and polarization, but it can also null desired signals if the jammer's signal direction is very close to that of the GPS satellite signals [12].

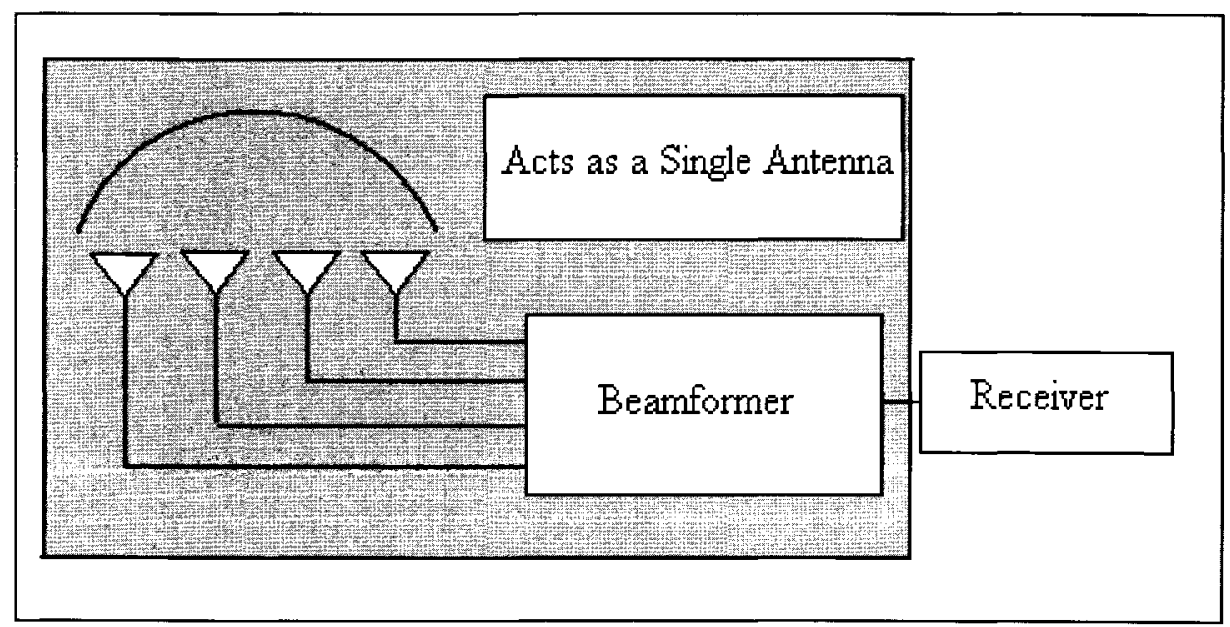

Figure 3.6: CRPAs Main Parts [12]

The rule here is that a CRPA with $\mathrm{N}$ antenna elements can steer nulls to the directions of $\mathrm{N}-1$ jammers. Normally CRPAs using this technique are made of a single beamformer, which can be easily cascaded with the GPS receivers. There are several advantages/disadvantages of simple null-steering over more complicated null/beamsteering. The advantages include:

- Operating without the knowledge of the GPS signal's direction of arrival (DOA), and independently from the GPS receiver core.

- A single beamformer results in a less complicated antenna electronics unit, which can be cascaded with any GPS receiver.

- No sensitivity to phase and amplitude errors associated with the antenna array, and no need for information regarding the array geometry. 
The main disadvantages of simple null-steering techniques include:

- The chance of canceling the desired signal because the pattern nulls can attenuate the nearby GPS signals.

- $\quad$ No additional gain in SNR over a single element antenna in the absence of a jammer, while beam-steering can provide up to $\mathrm{N} \mathrm{dB}$ gain.

- No guarantee of preserving the phase and amplitude of the desired GPS signal, which means they can't be use for applications requiring precise carrier phase measurements.

Multi-element antenna techniques mentioned above, are summarized in Table 3.2 on the next page.

The antenna array is a crucial part of any CRPA, and normally consists of more than 4 elements in a two dimensional plane, which enables the null-steering unit to create a null in both azimuth and elevation. Having more elements results in deeper nulls, decreasing the chance of eliminating the nearby GPS signals. Each antenna element needs to cover both the L1 and L2 carrier frequencies in addition to the new L5 frequency band, with at least $20 \mathrm{MHz}$ of bandwidth to cover the complete spectrum of $\mathrm{P}(\mathrm{Y})$ code. An important and limiting factor regarding the array design is the element spacing, which should ideally be half a wavelength of the carrier frequency. Smaller element spacing will result in reduced angular resolution of the array, resulting in wider nulls in the jammer's direction [11], [12]. 


\begin{tabular}{|c|c|c|c|c|}
\hline Technique & Implementation & Performance & Size & Cost \\
\hline $\begin{array}{l}\text { Switched } \\
\text { FRPA }\end{array}$ & $\begin{array}{l}\text { - Requires Several Antennas } \\
\text { - Requires electronic unit } \\
\text { for antenna switching }\end{array}$ & $\begin{array}{l}\text { - Provides additional anti-jam } \\
\text { against several low elevation } \\
\text { jammers } \\
\text { - Depends on the jammer } \\
\text { DOA } \\
\text { - Depends on the orientation } \\
\text { and radiation pattern of each } \\
\text { FRPA } \\
\text { - Limited or no flexibility in } \\
\text { adjusting the overall } \\
\text { radiation pattern }\end{array}$ & Small & Low \\
\hline $\begin{array}{l}\text { Multi- } \\
\text { element } \\
\text { Canceller }\end{array}$ & $\begin{array}{l}\text { - Requires at least two } \\
\text { elements } \\
\text { - Requires an electronic unit } \\
\text { to combine the signals } \\
\text { from primary and auxiliary } \\
\text { element }\end{array}$ & $\begin{array}{l}\text { - Mainly used for Aircraft } \\
\text { applications, canceling the } \\
\text { low elevation jammers } \\
\text { - Well separated GPS and } \\
\text { jammer signal results in } \\
\text { good cancellation of jammer } \\
\text { with no impact on GPS } \\
\text { signal } \\
\text { - Very sensitive to altitude }\end{array}$ & Large & High \\
\hline $\begin{array}{l}\text { Null- } \\
\text { Steering } \\
\text { CRPA }\end{array}$ & $\begin{array}{l}\text { - Requires large and } \\
\text { expensive antenna array } \\
\text { - Requires an expensive and } \\
\text { complicated antenna } \\
\text { electronics }\end{array}$ & $\begin{array}{l}\text { - Provide very good anti-jam } \\
\text { against multiple broadband } \\
\text { jammers } \\
\text { - Provides jammer elimination } \\
\text { in any direction by steering } \\
\text { null at that direction } \\
\text { - The nulls reduce the satellite } \\
\text { visibility and may cancel the } \\
\text { GPS signal as well }\end{array}$ & Large & High \\
\hline
\end{tabular}

Table 3.2: Multi-Element Techniques Summary 
Antenna spacing of more than half a wavelength creates ambiguity and additional unwanted nulls. The array is called a standard Array when the spacing of the elements is exactly half a wavelength. Due to this spacing constraint, the size of the antenna unit becomes very large in many military applications. The effects of changes in element spacing will be discussed in details in Chapter 4.

Some designs incorporate a reference antenna with a few auxiliary ones; the reference antenna picking up the GPS signal, while the auxiliary antennas estimate the jammer and try to cancel it from the signal received from the reference element. In the absence of any jammer, all the auxiliary antennas are disabled; with only the reference element being responsible for the performance of the antenna (therefore this element should have high gain). Some designs do not include a reference element and use the sum of all the elements' signals as the reference. The advantage of this type of design is that it maximizes the gain of the antenna unit when there is no jammer present [12].

\subsubsection{Null-Steering CRPAs}

In CRPAs the radiation pattern of the antenna array is controlled by the complex weights (e.g $\mathbf{W}_{\mathrm{i}}$ ) associated with the phase and amplitude, which are provided by the beamformer algorithm. This adaptive algorithm, currently implemented in digital technology, is responsible for continuously adapting the weights for the current jamming scenario. These digital beamformers have more flexibility and less sensitivity to tolerances of analog components, and are also much simpler to implement. 
The first step to design a null steering algorithm is to define an optimality criterion, which is described as follow: minimizing the output power of the array subject to one of the weights being fixed or clamped. The reference antenna has the fixed weight, while the auxiliary ones are used to find the minimum power. Optimizing this criterion leads to having nulls in the jammer's direction and reasonable gain in other directions. Figure 3.7 shows the comparison between FRPA's pattern and CRPA null steering pattern.

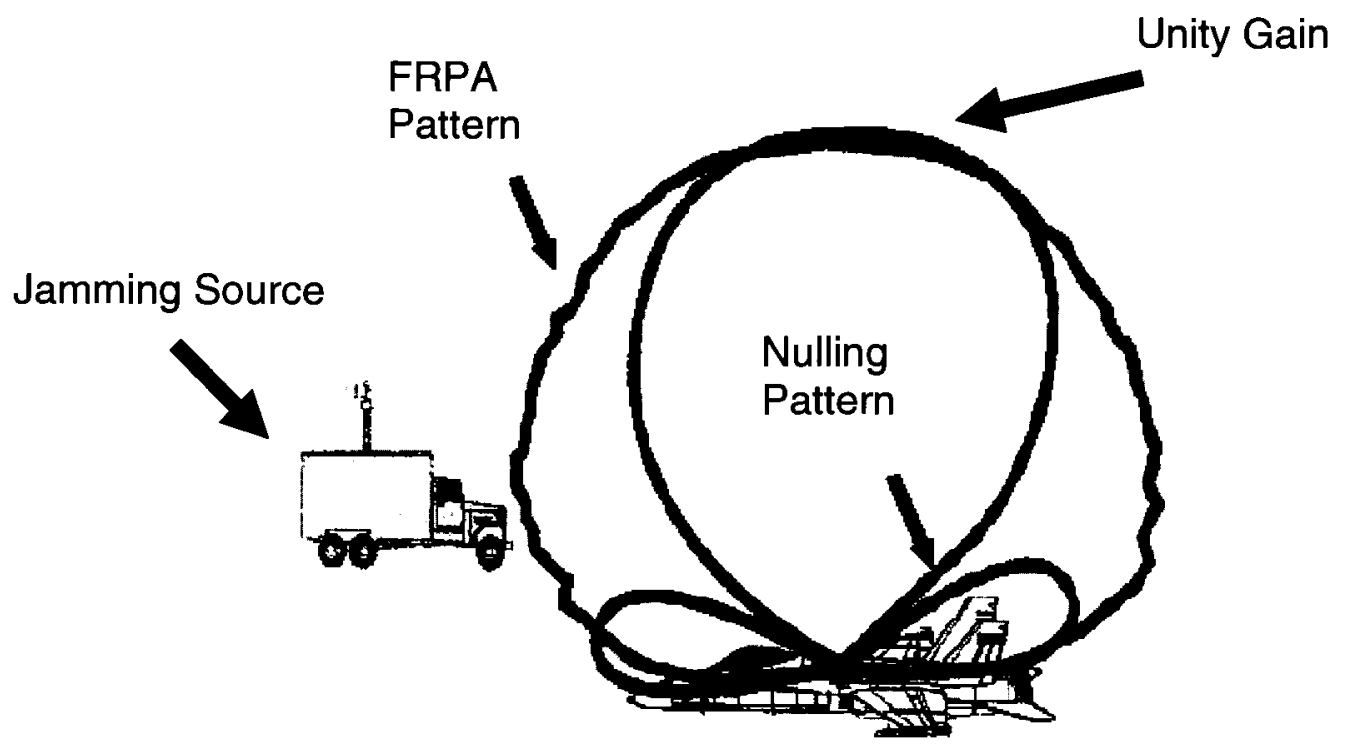

Figure 3.7: Null-Steering Performance versus FRPAs

The null steering algorithm and performance will be the discussed in detail in the next chapter, starting with the linear arrays and building up the case for more complex planar arrays. 


\subsection{Conclusion}

Anti-jamming techniques can be as simple as a front-end analog filtering to very complicated anti-spoofing techniques in high-end military receivers. The developing cost and size of anti-jamming devices increase as the hostility of the environment increases. In many scenarios, the high-end models are simply too bulky to carry around, which limits their use. In many cases, the high cost simply results in abandoning the anti-jamming techniques.

These limiting factors give the simpler techniques, such as null-steering algorithms, the chance to be deployed in many application having a low dynamic environment with only a limited number of jamming/interference sources. This is the reason why null-steering algorithms using planar arrays and the effect of number of elements in the array were chosen to study in this thesis. 


\section{Chapter 4: Array Beam Pattern Processing}

\subsection{Introduction}

As mentioned earlier, a null-steering beamformer can be more useful in many environments, where cost and size are the most important factors. These algorithms can effectively cancel multiple numbers of broadband jammers by simply pointing nulls at their direction. The down side is that if the jammer is located too close to the direction of the desired GPS signal, it may also eliminate the GPS signal and may cause loss of navigational data. This is not the main issue in this research since we are only concerned with jammers on low elevation angles.

To be able to study the null-steering algorithms and their effects on the array's beam pattern, it is essential to start with simple linear arrays with uniform spacing and weighting. Then the optimum beam pattern using the Dolph-Chebyshev criteria will be discussed considering the effect of imposing nulls on the beam pattern. In the next step, the case of the linear array is extended to planar arrays. The algorithms for null-steering are then developed in MATLAB and the simulation results are provided. The null+beam steering is also discussed and the simulation results are presented. The last part of this chapter contains the discussion on the optimum beam pattern in the presence of interference. The materials covered in this chapter are considered as an extension to the classical array theory, which has been discussed in many books and articles [14], [16], [17], [18], [19]. 


\subsection{Linear Array Beam Pattern}

\subsubsection{Linear Arrays Overview}

The spherical coordinate system, as shown in Figure 4.1, is used in this section as the system of interest. This helps to define the beam pattern in three dimensions. The angle in the azimuth plane is defined by $\phi$, and the angle in the elevation plane is defined by $\theta$. The relationship between spherical and rectangular coordinates is as follow:

$$
\begin{aligned}
& x=r \sin \theta \cos \phi \\
& y=r \sin \theta \sin \phi \\
& z=r \cos \theta
\end{aligned}
$$

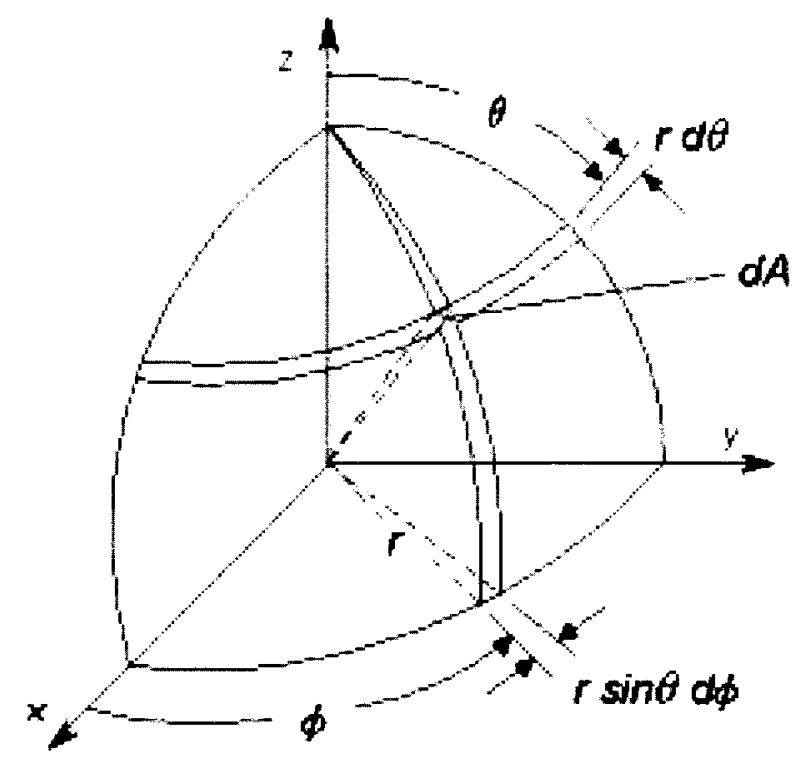

Figure 4.1: Spherical Coordinate System 
A Uniform Linear Array, or simply called a ULA (Figure 4.2) is a linear array with $\mathrm{N}$ number of elements equally spaced (in this chapter along the $z$-axis). Position of a linear array's elements, $\mathrm{p}_{\mathrm{zn}}$, is defined as follow:

$$
p_{z n}=\left(n-\frac{N-1}{2}\right) d, n=0,1, \ldots, N-1
$$

and

$$
p_{x n}=p_{y n}=0
$$

Defining the direction cosines with respect to each axis as

$$
\vec{u}=\left[\begin{array}{c}
u_{x}=\sin \theta \cos \phi \\
u_{y}=\sin \theta \sin \phi \\
u_{z}=\cos \theta
\end{array}\right],
$$

and the unit vector as

$$
\vec{a}=-\vec{u}
$$

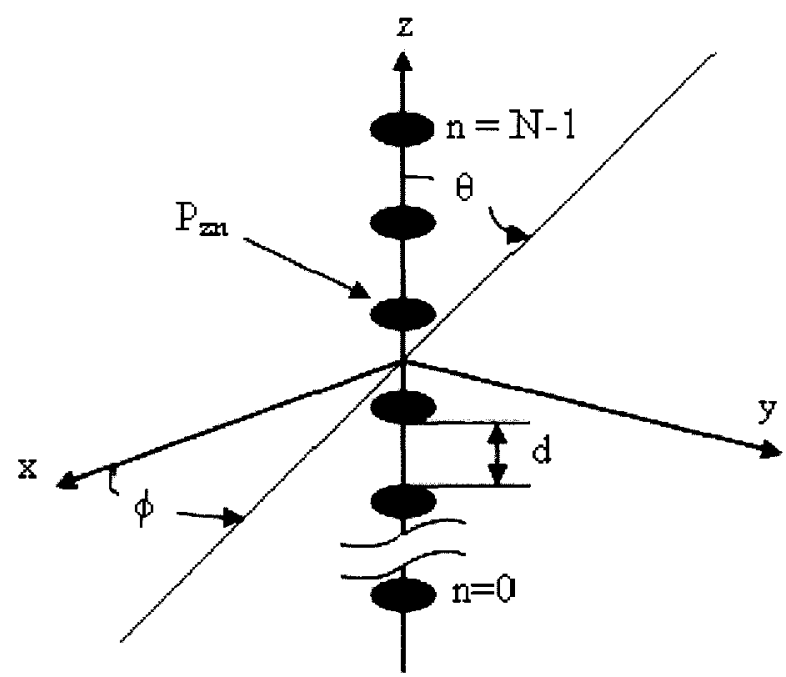

Figure 4.2: ULA along $\mathrm{z}$-axis 
Now we can define the Array Manifold Vector (AMV) as

$$
\vec{v}_{k}(\vec{k})=\left[\begin{array}{c}
e^{-j \vec{k}^{T} \vec{p}_{0}} \\
e^{-j \vec{k}^{T} \vec{p}_{1}} \\
\hdashline e^{-j \vec{k}^{T} \vec{p}_{N-1}}
\end{array}\right]
$$

where the subscript $k$ denotes that the argument is in $k$-space and the wavenumber vector is defined as follow

$$
\vec{k}=-\frac{2 \pi}{\lambda} \vec{a}=-\frac{2 \pi}{\lambda}\left[\begin{array}{c}
\sin \theta \cos \phi \\
\sin \theta \sin \phi \\
\cos \theta
\end{array}\right]
$$

substituting (4.2) and (4.3) in (4.6) we obtain

$$
\vec{v}_{k}\left(k_{z}\right)=\left[\begin{array}{l:l:l:l}
e^{j\left(\frac{N-1}{2}\right) k_{z} d} & e^{j\left(\frac{N-1}{2}-1\right) k_{z} d} & \ldots & e^{-j\left(\frac{N-1}{2}\right) k_{z} d}
\end{array}\right]^{T}
$$

with

$$
k_{z}=\frac{2 \pi}{\lambda} \cos \theta=k_{0} \cos \theta
$$

The elements of the antenna array spatially pick up the signal field, which yields a set of signal denoted by the following vector

$$
\vec{f}(t, \vec{p})=\left[\begin{array}{c}
f\left(t, \vec{p}_{0}\right) \\
f\left(t, \vec{p}_{1}\right) \\
\cdot \\
\cdot \\
\cdot \\
f\left(t, \vec{p}_{N-1}\right)
\end{array}\right]
$$

To obtain the output of array $y(t)$, the output of each element is processed by a linear, time invariant filter with impulse response $h_{n}(\tau)$ and the results are then added. This processor is illustrated in Figure 4.3. 
The output can be define in vector notation as

$$
y(t)=\int_{-\infty}^{\infty} \vec{h}^{T}(t-\tau) \vec{f}(\tau, \vec{p}) d \tau,
$$

where

$$
\vec{h}(\tau)=\left[\begin{array}{c}
h_{0}(\tau) \\
h_{1}(\tau) \\
\cdot \\
\cdot \\
\cdot \\
h_{N-1}(\tau)
\end{array}\right]
$$

Further we can define (4.11) in the transform domain as

$$
Y(\omega)=\int_{-\infty}^{\infty} y(t) e^{-j \omega t} d t=\vec{H}(\omega) \vec{F}(\omega),
$$

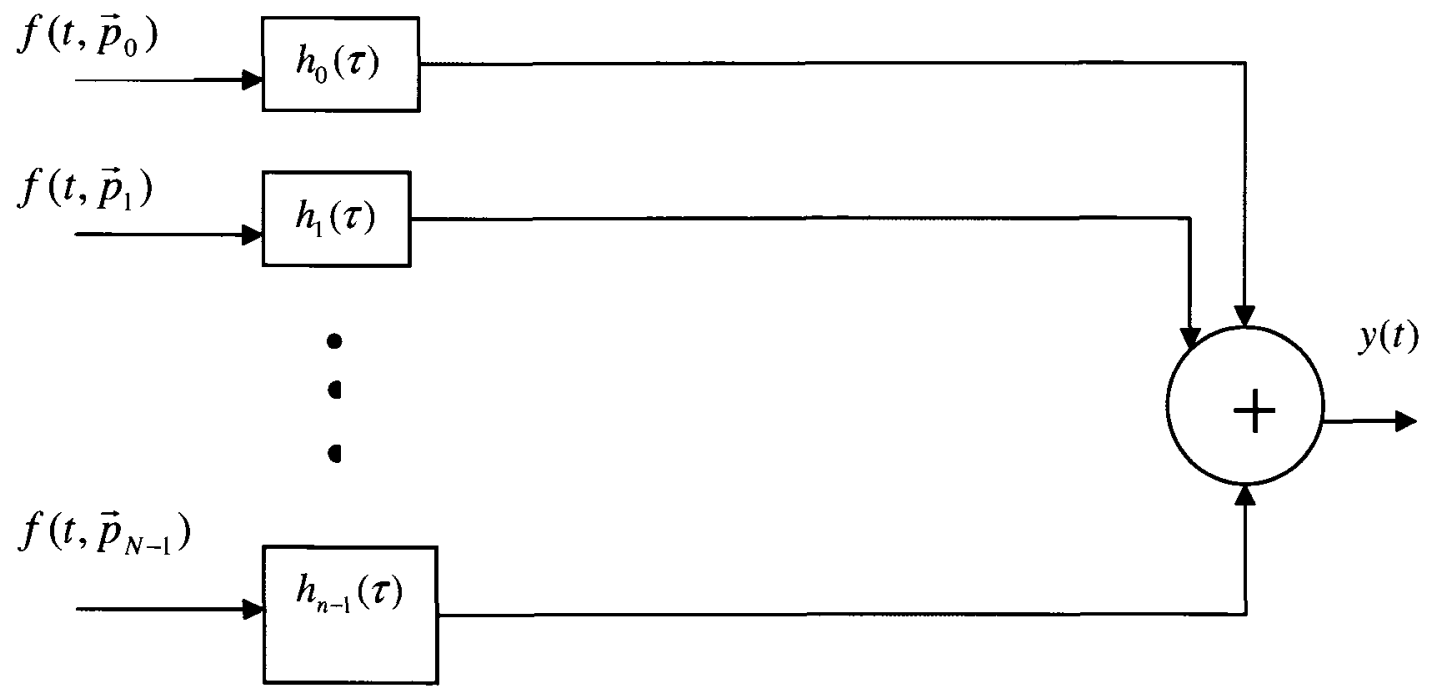

Figure 4.3: Linear Array processing 
Now the output of each element is shifted so that all of them are aligned in time, then the signals are added. There should be a normalization of $1 / \mathrm{N}$ so the output is exactly $f(t)$. This means, $h_{n}(\tau)$ can be defined as

$$
h_{n}(\tau)=\frac{1}{N} \delta\left(\tau+\tau_{n}\right)
$$

or in the frequency domain as

$$
H^{T}(\omega)=\frac{1}{N} \vec{v}_{\vec{k}}^{H}\left(\vec{k}_{s}\right)
$$

Where $\vec{v}_{\vec{k}}\left(\vec{k}_{s}\right)$ is defined in (4.8).

Usually, for the shifting of the signals a delay is used in each channel, which makes the operation more tractable. This type of processor, shown in Figure 4.4, is called delay-and-sum beamformer or the conventional beamformer.

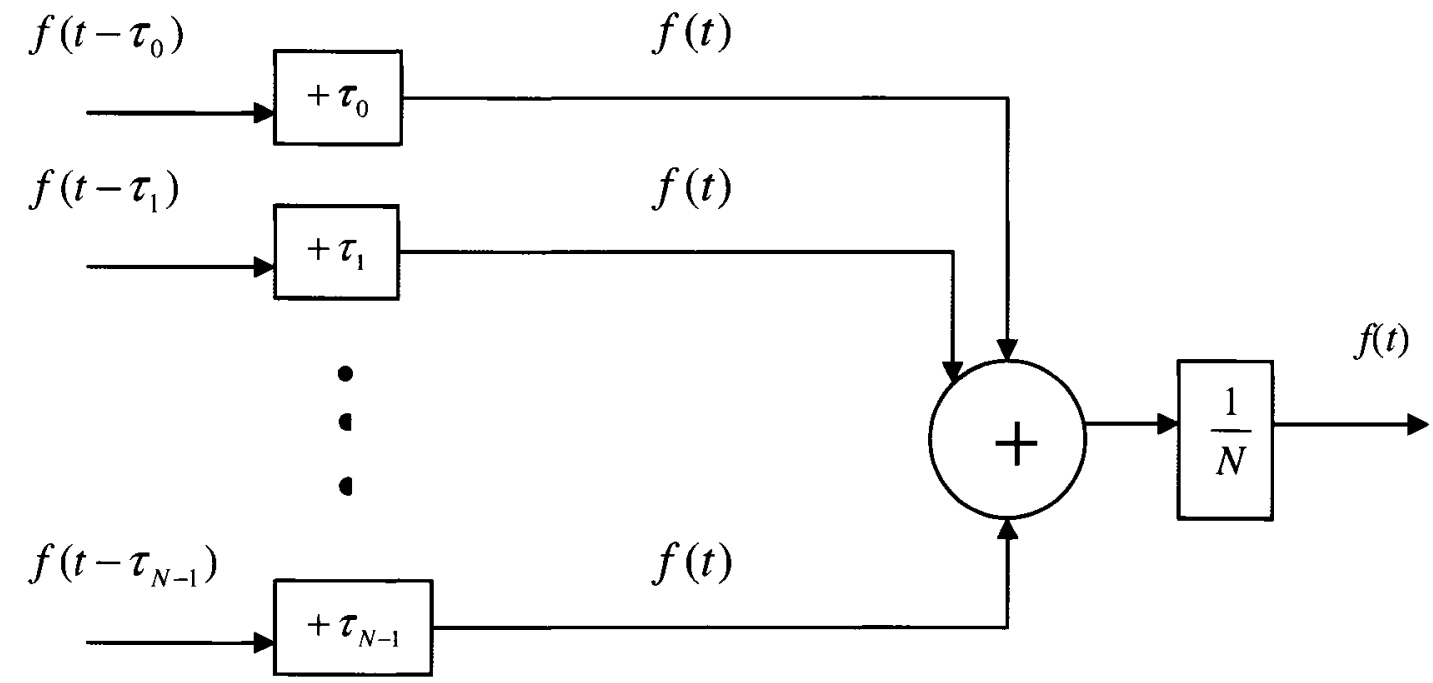

Figure 4.4: The Conventional Beamformer Processor 
Considering plane waves of the form,

$$
\vec{f}(t, \vec{p})=e^{j \omega t} \vec{v}_{\vec{k}}(\vec{k}),
$$

the response of processor (4.11) to above plane wave is

$$
y(t, \vec{k})=\vec{H}^{T}(\omega) \vec{v}_{\vec{k}}(\vec{k}) e^{j \omega t},
$$

or in frequency domain as

$$
Y(\omega, \vec{k})=\vec{H}^{T}(\omega) \vec{v}_{\vec{k}}(\vec{k}) .
$$

where $\omega$ is a single frequency of the input signal.

The above equation is called the frequency-wavenumber response function of the array. It simply gives the complex response of the array to an input plane wave with wavenumber $\vec{k}$ and temporal frequency of $\omega$. Therefore the beam pattern of an array is defined as the frequency-wavenumber response function evaluated versus the direction, as follows:

$$
B(\omega: \theta, \phi)=\left.Y(\omega, \vec{k})\right|_{\vec{k}=\frac{2 \pi}{\lambda} \vec{a}(\theta, \phi)},
$$

This is simply the function evaluated on a sphere of radius $2 \pi / \lambda$, where $\vec{a}(\theta, \phi)$ is a unit vector with coordinate angles of $\theta, \phi$.

Now we can define the complex weight vector as

$$
\vec{w}^{H}=\left[\begin{array}{lllll}
w_{0}^{*} & w_{1}^{*} & \cdot & w_{N-1}^{*}
\end{array}\right]=\vec{H}^{T}(\omega),
$$


Now back to the case for the uniform linear array located along the z-axis. For the output of the array we have the following:

$$
Y\left(\omega, k_{z}\right)=\vec{w}^{H} \vec{v}_{\vec{k}}\left(k_{z}\right)=\sum_{n=0}^{N-1} \omega_{n}^{*} e^{-j\left(n-\frac{N-1}{2}\right) k_{z} d} .
$$

where

$$
k_{z}=\frac{2 \pi}{\lambda} u_{z}=\frac{2 \pi}{\lambda} \cos \theta
$$

It can also be written in $\psi$-space as

$$
Y_{\psi}(\psi)=e^{-j \frac{N-1}{2} \psi} \sum_{n=0}^{N-1} \omega_{n}^{*} e^{j n \psi},
$$

where

$$
\psi=-k_{z} d=\frac{2 \pi}{\lambda} \cos \theta \cdot d
$$

The array manifold vector is normally written in $\psi$-space too:

$$
\vec{v}_{\psi}(\psi)=\left[\begin{array}{l:l:l:l}
e^{j\left(\frac{N-1}{2}\right) \psi} & e^{j\left(\frac{N-1}{2}-1\right) \psi} & \ldots & e^{-j\left(\frac{N-1}{2}\right) \psi}
\end{array}\right]^{T}
$$

Although both (4.20) and (4.22) are between $-\infty$ and $\infty$, only signals in the region $-\pi \leq \theta \leq \pi$ or $-1 \leq u \leq 1$ are considered. This region is called "the visible region" of an array and in $\psi$-space is defined as follow

$$
-\frac{2 \pi d}{\lambda} \leq \psi \leq \frac{2 \pi d}{\lambda}
$$


As mentioned earlier for the Uniformly Weighted Linear Array (UWLA), all the elements have the same weighting as

$$
\vec{w}=\frac{1}{N} \overrightarrow{1},
$$

where $\overrightarrow{1}$ is the $N \times 1$ unity vector, this results in the following for the output of the array

$$
Y_{\psi}(\psi)=\frac{1}{N} e^{-j \frac{N-1}{2} \psi} \sum_{n=0}^{N-1} e^{j n \psi}=\frac{1}{N} e^{-j \frac{N-1}{2} \psi}\left[\frac{1-e^{j N \psi}}{1-e^{j \psi}}\right]
$$

This is equal to

$$
Y_{\psi}(\psi)=\frac{1}{N} \frac{\sin \left(N \frac{\psi}{2}\right)}{\sin \frac{\psi}{2}}, \quad-\infty\langle\psi<\infty
$$

Considering the visible region defined earlier, we have the following equation for the beam pattern of the UWLA:

$$
B_{\psi}(\psi)=\frac{1}{N} \frac{\sin \left(N \frac{\psi}{2}\right)}{\sin \frac{\psi}{2}}, \quad-\frac{2 \pi}{\lambda}\left\langle\psi<\frac{2 \pi}{\lambda}\right.
$$

or similarly in $\theta$-space:

$$
\begin{aligned}
B_{\theta}(\theta) & =\frac{1}{N} \frac{\sin \left(\frac{N}{2} \cdot \frac{2 \pi}{\lambda} \cos \theta \cdot d\right)}{\sin \left(\frac{1}{2} \cdot \frac{2 \pi}{\lambda} \cos \theta \cdot d\right)} \\
& =\frac{1}{N} \frac{\sin \left(\frac{N \pi}{\lambda} \cos \theta \cdot d\right)}{\sin \left(\frac{\pi}{\lambda} \cos \theta \cdot d\right)}, \quad-\pi \leq \theta \leq \pi
\end{aligned}
$$


The following table shows some of the correspondences between the field of time-domain digital signal processing and array processing (space-domain signal processing). [15]

\begin{tabular}{|l|l|}
\hline Time-Domain Signal Processing & Space-Domain Signal Processing \\
\hline Sampling time interval $T$ & Sampling space interval $d$ \\
\hline Sampling rate $1 / T$ (sample/sec) & Sampling rate 1/d (sample/meter) \\
\hline Frequency $\Omega$ & Wavenumber $k$ \\
\hline Time-domain sampling $t_{n}=n T$ & Space-domain sampling $x_{n}=n d$ \\
\hline Digital frequency $\omega=\Omega T$ & Digital Wavenumber $\psi=k d$ \\
\hline Nyquist Interval $-\pi \leq \omega \leq \pi$ & Nyquist Interval $-\pi \leq \psi \leq \pi$ \\
\hline Sampling theorem $\Omega \leq \pi / T$ & Sampling theorem $k \leq \pi / d$ \\
\hline Spectral images & Grating lobes \\
\hline z-domain $z=e^{j \omega}$ & z-domain $z=e^{j \psi}$ \\
\hline Pure sinusoid $e^{j \omega_{0} n}$ & Narrow beam $e^{j \psi_{0} n}$ \\
\hline Frequency shifting & Array scanning \\
\hline Frequency response $Y(\omega)$ & Array factor $Y(\psi)$ \\
\hline
\end{tabular}

Table 4.1: Time-Domain and Space-Domain Dualities 
A polar plot of (4.30) can be seen in Figure 4.5 for a 4-element and an 8-element UWLA with spacings of half-wavelength (mainbeam towards $90^{\circ}$ or broadside), and Figure 4.6 shown the magnitudes of beam patterns of the arrays in $u$-space.
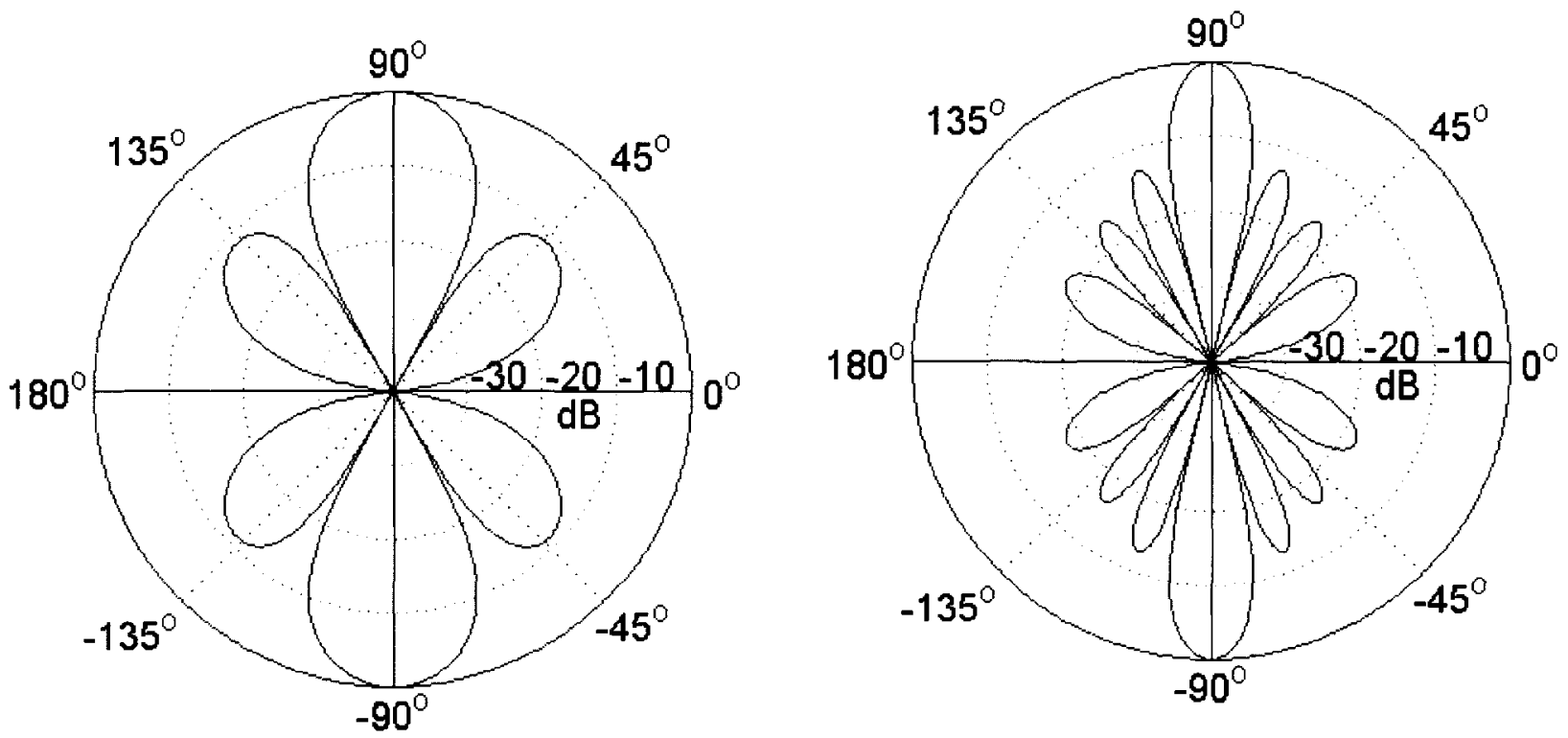

Figure 4.5: Angular Radiation Pattern of 4 and 8-element Array
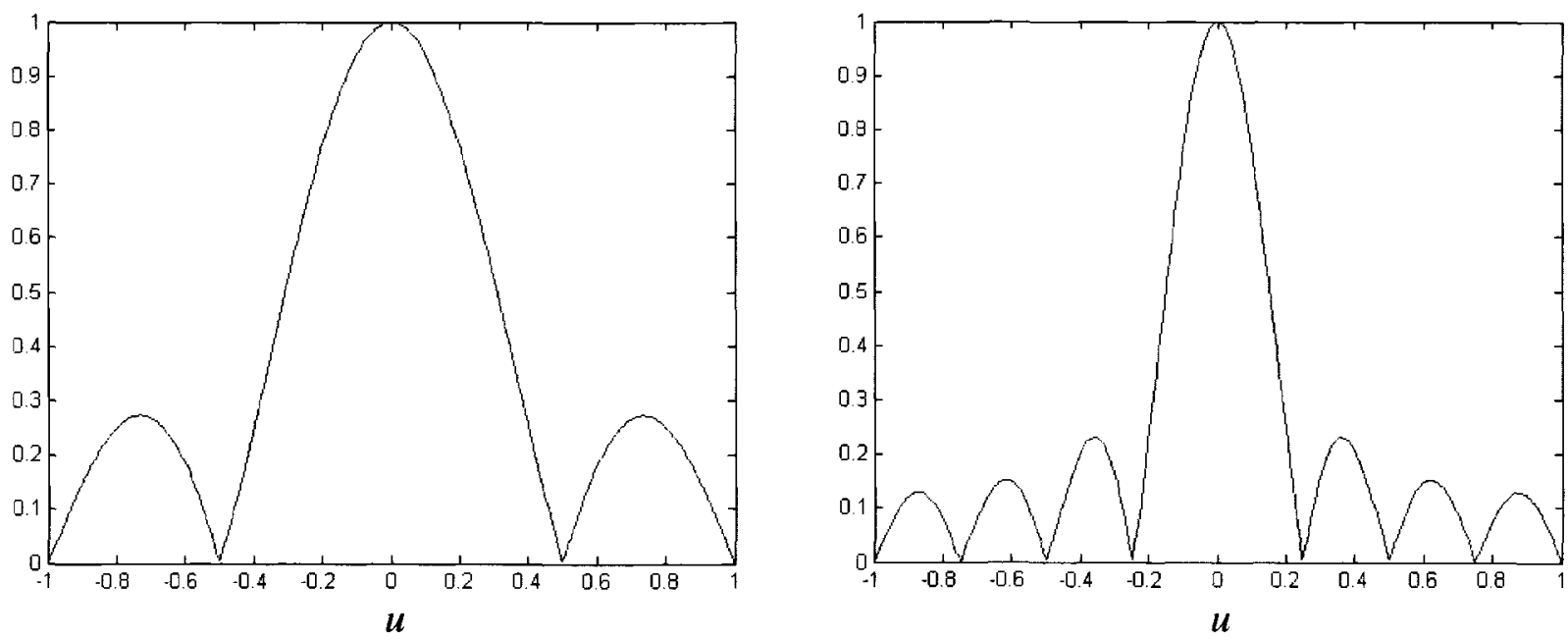

Figure 4.6: Array Factor for 4 and 8-element Array 
As mentioned earlier the standard element spacing is half of the wavelength of the desired signal. In the case of $d \geq \lambda / 2$, the array output $Y(\psi)$ will be repeated in the visible region (in $u$-space it is $-1 \leq u \leq 1$ ). This creates grating lobes, which are simply the mainlobes in undesired directions. This can be compared to spectral images when the Nyquist criterion is not met (undersampling in the time-domain waveform). In other words, grating lobes occur when we have the following in (4.29):

$$
\psi=n 2 \pi \text { or } u=n \frac{\lambda}{d}
$$

The grating lobes can be simply avoided by making sure the element spacing is less than a wavelength. Although to completely avoid any repetition in beam pattern, we must have $d \leq \lambda / 2$. Having spacing less than half-a-wavelength will widen the main lobe and decrease the number of sidelobes. The effect of array spacing can be seen in the following figures for an 8-element ULA.
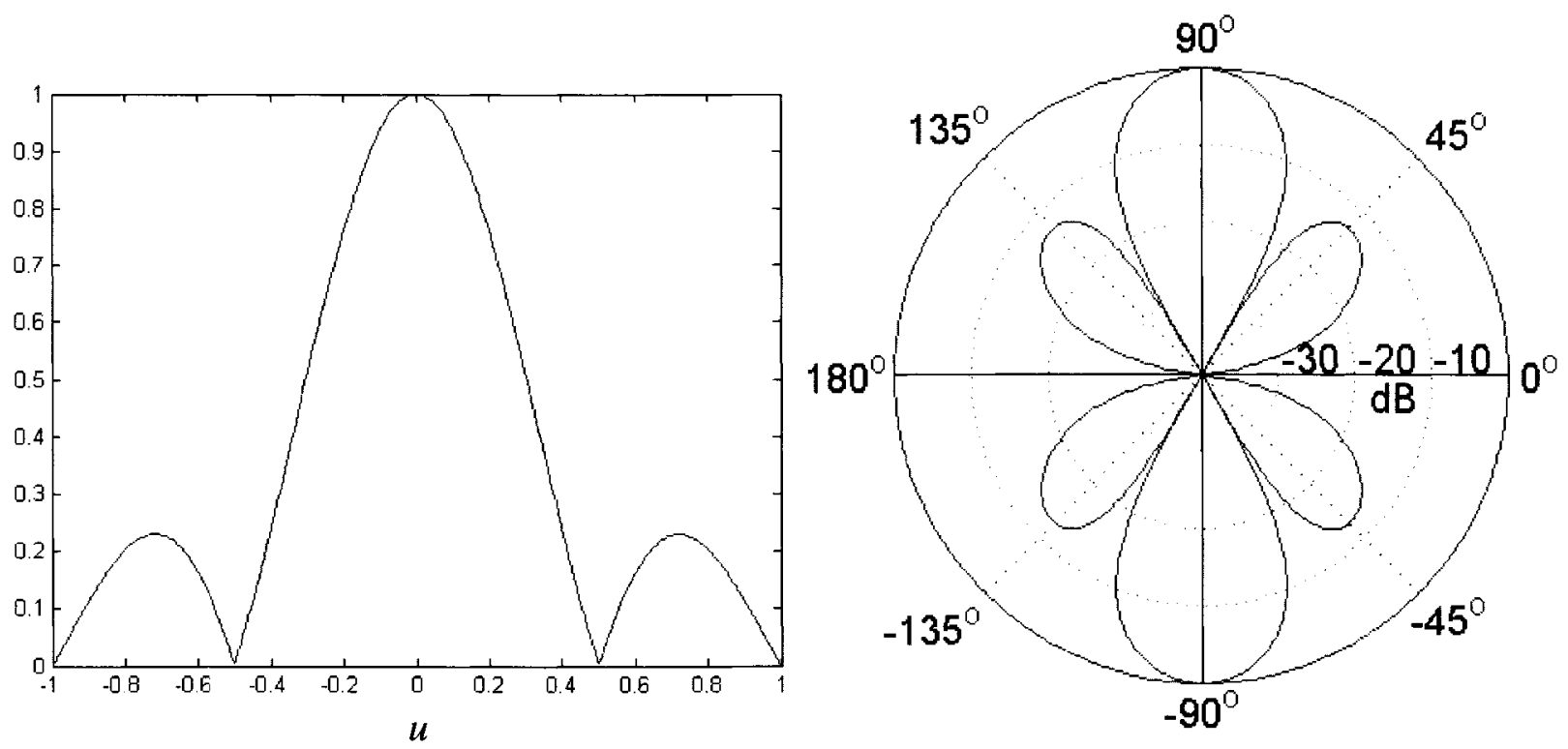

Figure 4.7: Effect of $\lambda / 4$ Element Spacing in $u$-space and $\theta$-space 

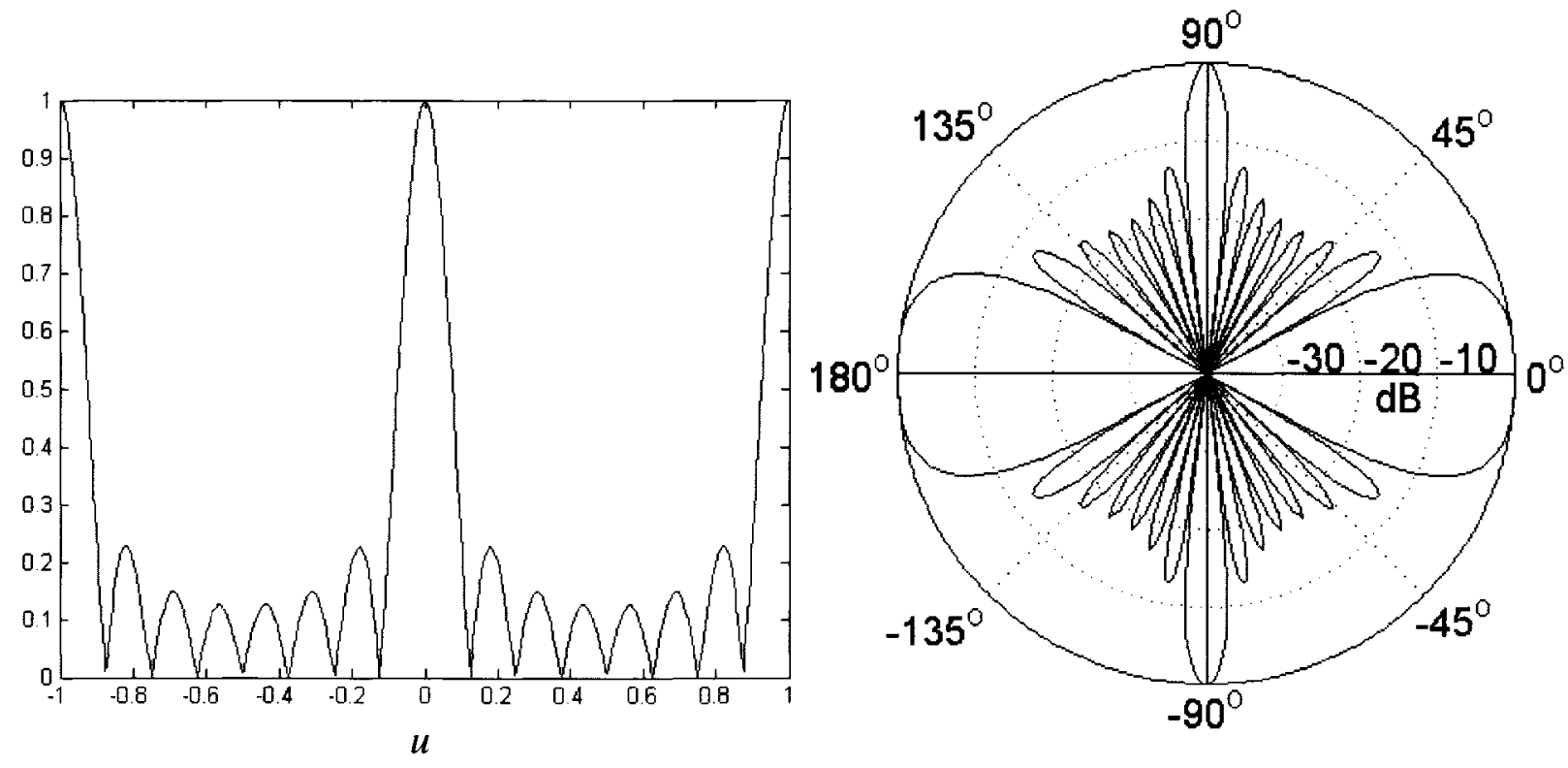

Figure 4.8: Effect of $\lambda$ Element Spacing in $u$-space and $\theta$-space
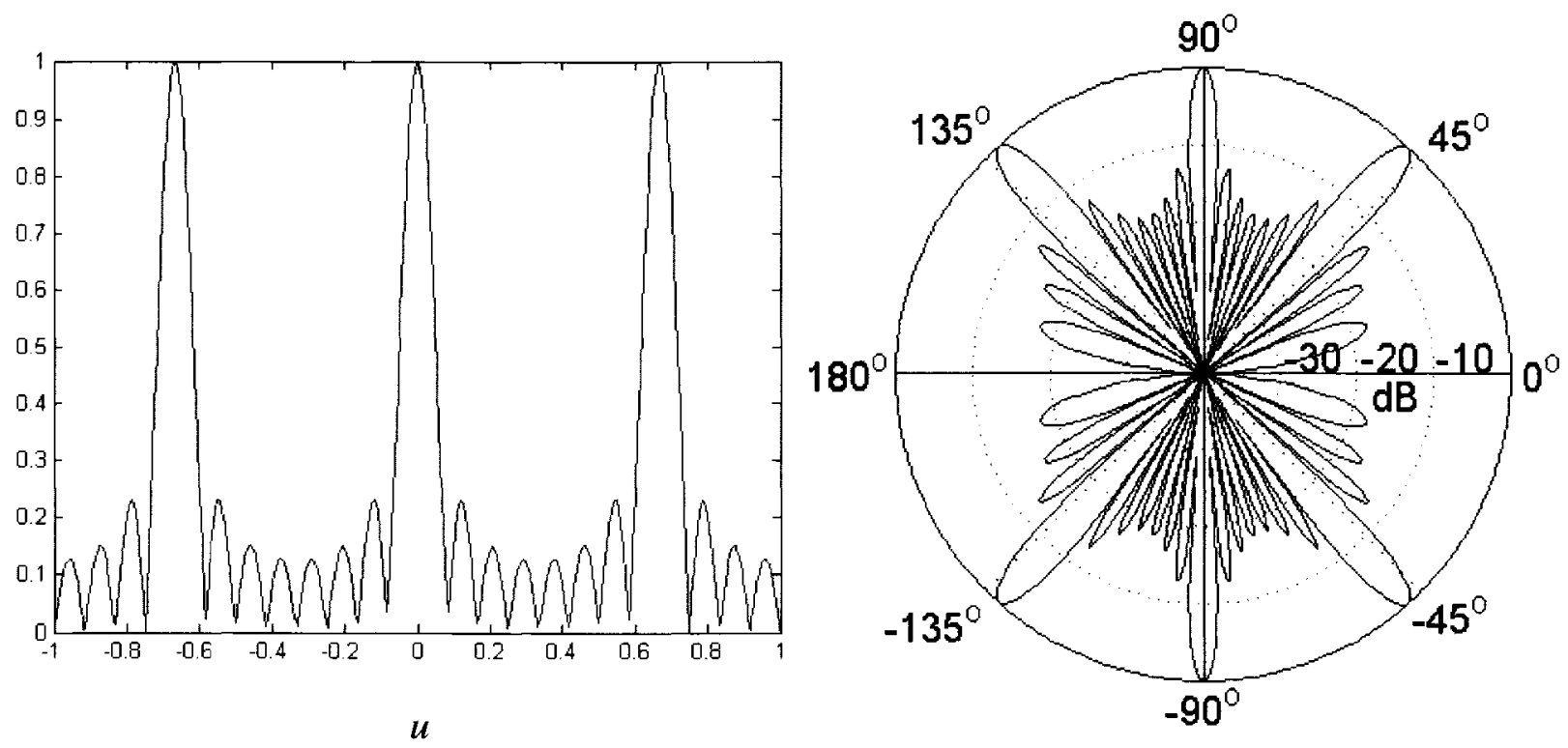

Figure 4.9: Effect of $\frac{3 \lambda}{2}$ Element Spacing in $u$-space and $\theta$-space

As can be seen, the mainlobe repeats itself and moves to the visible region as the element spacing increases beyond one wavelength. 


\subsubsection{Linear Array Beam Steering}

Now the goal is to steer the array's main response axis using electronic steering. As mentioned in the previous chapter the very high speed of today's digital signal processors, in addition to restrictions on mechanical steering, has resulted in extensive use of electronic steering in array processing applications. Considering the input defined in (4.15), we want to align the output so that $\vec{k}=\vec{k}_{S}$, where $\vec{k}_{S}$ is termed the steering direction. This can be achieved by using a $N \times N$ diagonal steering matrix in the following form,

$$
\vec{I}\left(\vec{k}_{S}\right)=\left[\begin{array}{cccccc}
e^{j \vec{k}_{S}^{T} \vec{p}_{1}} & 0 & \cdot & \cdot & \cdot & 0 \\
0 & e^{j \vec{k}_{S}^{T} \vec{p}_{2}} & . & \cdot & \cdot & 0 \\
\cdot & \cdot & \cdot & \cdot & \cdot & \cdot \\
\cdot & \cdot & \cdot & \cdot & \cdot & \cdot \\
0 & \cdot & \cdot & \cdot & e^{j \vec{k}_{S}^{T} \vec{p}_{N-1}} & 0 \\
0 & 0 & . & \cdot & 0 & e^{j \vec{k}_{S}^{T} \vec{p}_{N}}
\end{array}\right] .
$$

This results in the following output, which is simply moving the main response axis to the new direction $\vec{k}_{S}$,

$$
Y\left(\omega, \vec{k} \mid \vec{k}_{S}\right)=Y\left(\omega, \vec{k}-\vec{k}_{S}\right)
$$

Therefore for a ULA we have the beam pattern in $\psi$-space as

$$
B_{\psi}\left(\psi: \psi_{S}\right)=\frac{1}{N} \frac{\sin \left(N \frac{\psi-\psi_{S}}{2}\right)}{\sin \left(\frac{\psi-\psi_{S}}{2}\right)}
$$


in $u$-space as

$$
B_{u}\left(u: u_{S}\right)=\frac{1}{N} \frac{\sin \left[\frac{\pi N d}{\lambda}\left(u-u_{S}\right)\right]}{\sin \left[\frac{\pi d}{\lambda}\left(u-u_{S}\right)\right]}
$$

and in $\theta$-space as

$$
B_{\theta}\left(\theta: \theta_{S}\right)=\frac{1}{N} \frac{\sin \left[\frac{\pi N d}{\lambda}\left(\cos \theta-\cos \theta_{S}\right)\right]}{\sin \left[\frac{\pi d}{\lambda}\left(\cos \theta-\cos \theta_{S}\right)\right]}
$$

The following figures (Figure 4.10 and Figure 4.11) show the beam steering in both $u$-space and $\theta$-space for steering angles of $60^{\circ}$ and $30^{\circ}$ for a 4-element uniform weighted linear array.
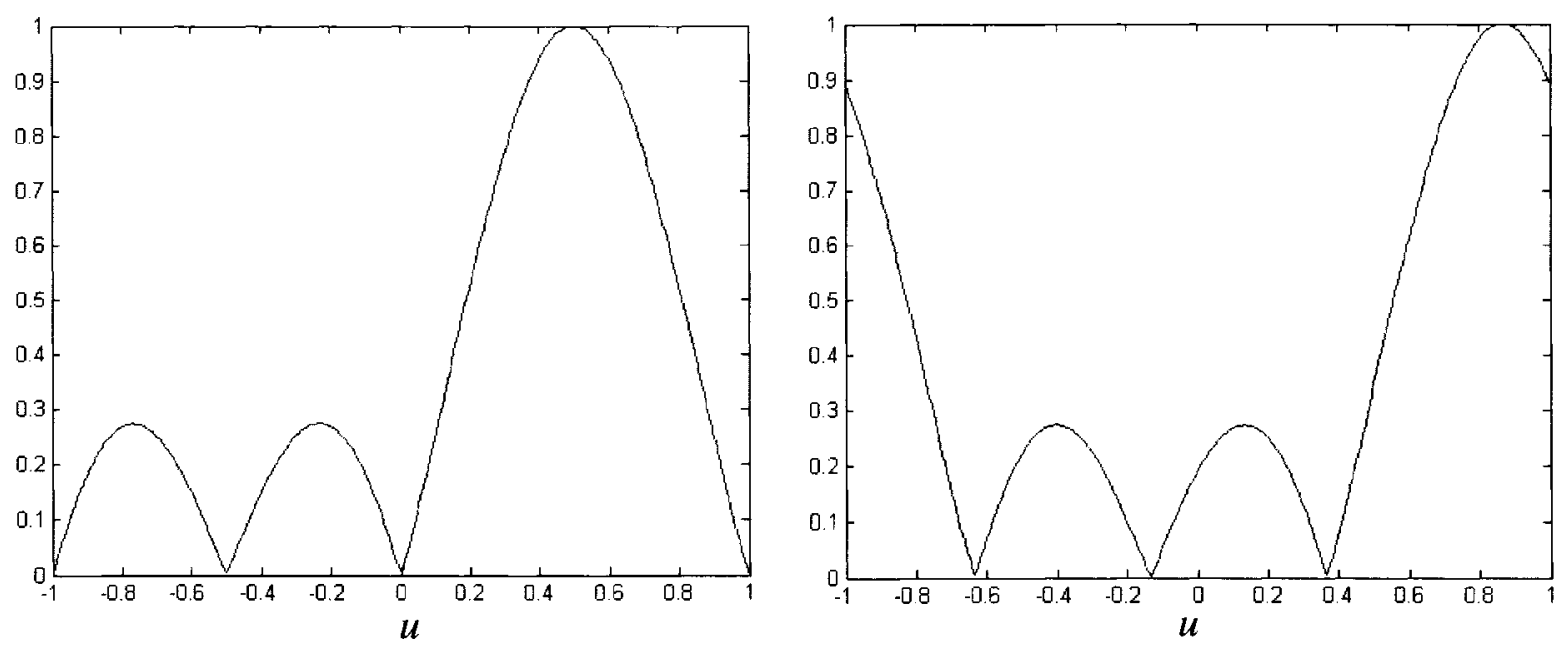

Figure 4.10: Steering Beam to $60^{\circ}$ and $30^{\circ}$ in $u$-space with a 4-element Array 

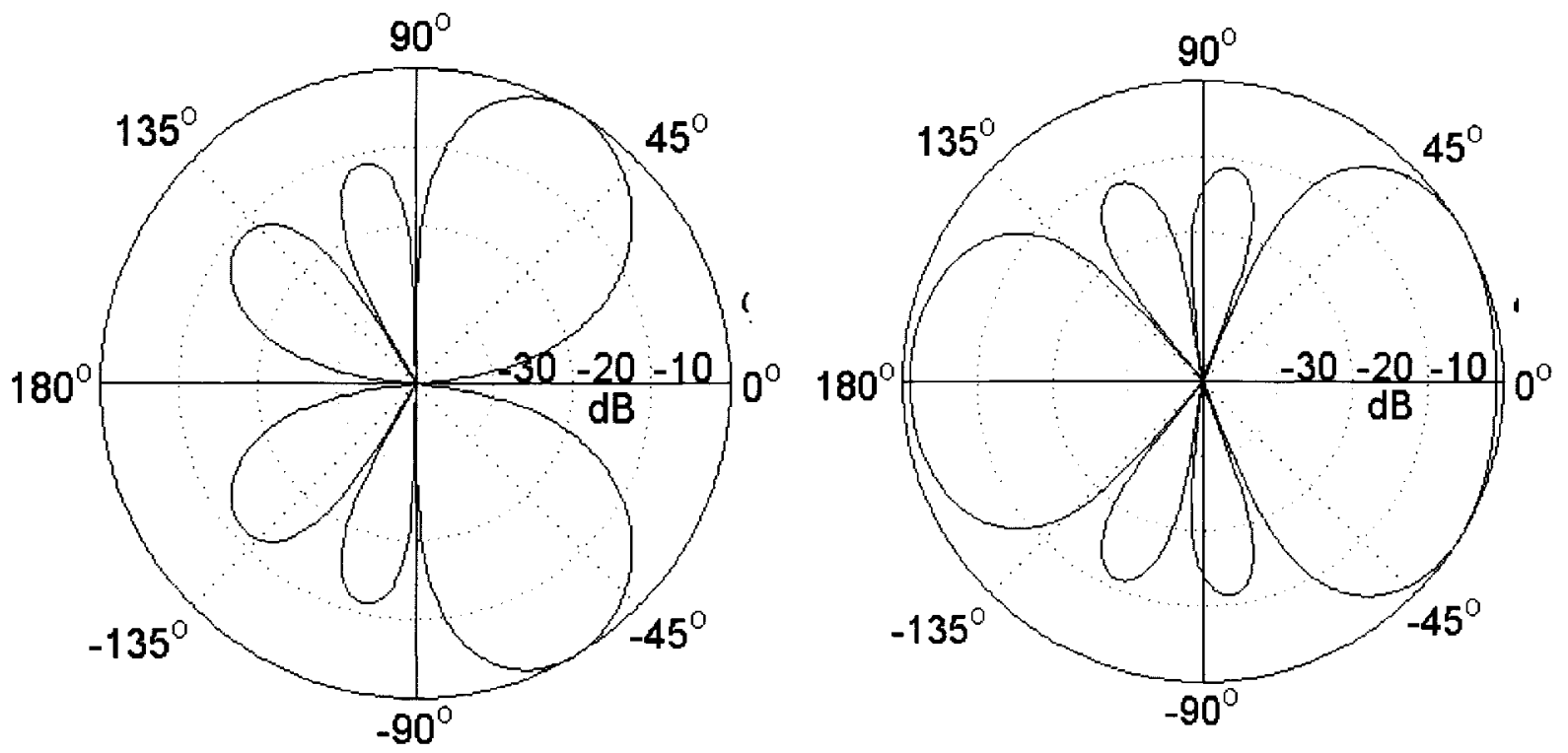

Figure 4.11: Steering Beam to $60^{\circ}$ and $30^{\circ}$ in $\theta$-space with a 4-element Array

Figure 4.12 shows the steering of the main beam to $60^{\circ}$ and $30^{\circ}$ in an UWLA with 8 elements in $\theta$-space. The change in the resolution of beam steering can be seen by comparing this figure with the previous figures.
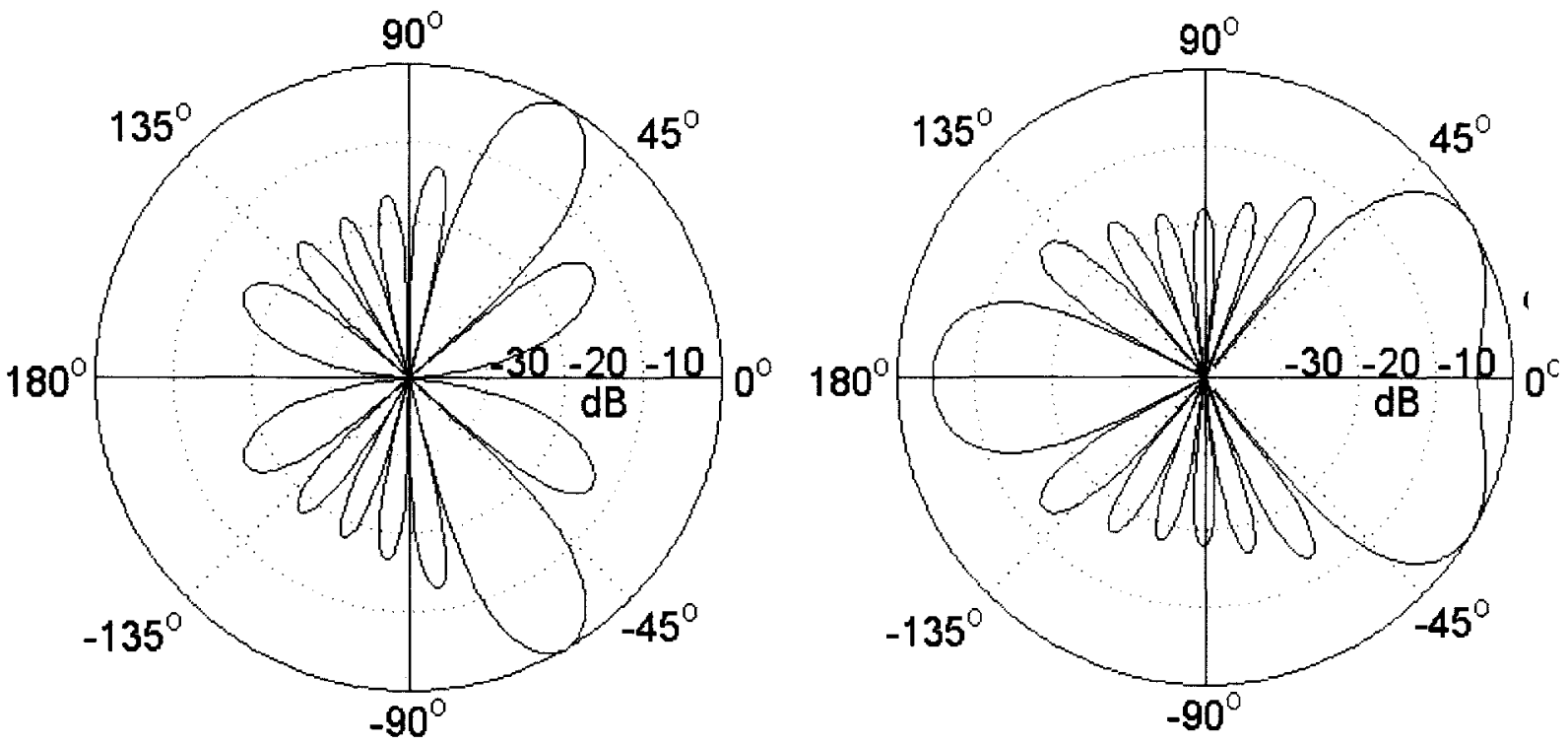

Figure 4.12: Steering Beam to $60^{\circ}$ and $30^{\circ}$ in $\theta$-space with an 8-element Array 


\subsubsection{Dolph-Chebyshev Weighting for Linear Arrays}

Almost all weighting techniques result in having the largest sidelobe level near the mainlobe. However by using some techniques the level of the sidelobes can be set to a fixed level, at the expense of increase in mainlobe width. This means that for a desired sidelobe level $R_{d B}$, the minimum mainlobe width can be achieved by weighting window that sets all the sidelobes' level to $R_{d B}$. In other words, for a given maximum mainlobe width, the maximum sidelobe attenuation can be achieved by having equal sidelobe levels. For this purpose the optimum weighting window is the Dolph-Chebyshev window (Originally implemented in [22]), which is based upon the Chebyshev polynomials. The $m$-th order Chebyshev polynomial $T_{m}(x)$, is defined as

$$
T_{m}(x)=\cos \left(m \cos ^{-1}(x)\right),
$$

If $|x|>1$, then the arc-cosine becomes imaginary and the (4.36) will be written as a hyperbolic cosine:

$$
T_{m}(x)=\cosh \left(m \cosh ^{-1}(x)\right),
$$

Expanding the quantity $\cos (m \theta)$ as a polynomial in powers of $\cos \theta$, and using the trigonometric identities results in the coefficients of the power of $x$ of the Chebyshev polynomial for particular values of $m$ (the first 5 polynomials shown below):

$$
\begin{aligned}
& T_{0}(x)=1 \\
& T_{1}(x)=x \\
& T_{2}(x)=2 x^{2}-1 \\
& T_{3}(x)=4 x^{3}-3 x \\
& T_{4}(x)=8 x^{4}-8 x^{2}+1
\end{aligned}
$$


The Dolph-Chebyshev array factor is defined by the Chebyshev polynomial of degree $N-1$ ( $\mathrm{N}$ is the number of elements in the array). With $x=x_{0} \cos \left(\frac{\psi}{2}\right)$, this can be written as follow

$$
W(\psi)=T_{N-1}(x), \quad x=x_{0} \cos \left(\frac{\psi}{2}\right)
$$

when $x=x_{0}$, we have the factor for the main beam

$$
W_{\text {main_ } \text { heam }}=T_{N-1}\left(x_{0}\right) \text {, }
$$

This is simply equal to $R$ (absolute unit), the ratio between the mainlobe level and sidelobe level in a normalized beam pattern.

$$
R=\frac{W_{\text {main_lobe }}}{W_{\text {side_lobe }}}=10^{R_{d \mathrm{~dB}} / 20}
$$

or in $\mathrm{dB}$,

$$
R_{d B}=20 \log _{10}(R)
$$

combining (4.37), (4.40), and (4.41), we have the following:

$$
R=T_{N-1}\left(x_{0}\right)=\cosh \left((N-1) \cosh ^{-1}\left(x_{0}\right)\right),
$$

From (4.43), the $x_{0}$ can be calculated as

$$
x_{0}=\cosh \left(\frac{\cosh ^{-1}(R)}{N-1}\right) \text {, }
$$


So the beam pattern is

$$
B(\psi)=\frac{T_{N-1}\left(x_{0} \cos \left(\frac{\psi}{2}\right)\right)}{R},
$$

Now the $N-1$ zeros of $T_{N-1}(x)$ defined as $x_{p}$ and can be found as follow:

$$
\begin{aligned}
& T_{N-1}(x)=\cos \left((N-1) \cos ^{-1}(x)\right)=0 \\
& \Rightarrow x_{p}=\cos \left(\frac{(2 p-1) \pi}{2(N-1)}\right), \quad p=1,2, \ldots, N-1,
\end{aligned}
$$

using $w_{p}=\frac{x_{p}}{x_{0}}=\cos \left(\frac{\psi_{p}}{2}\right)$, gives the zeros in $\psi$-space,

$$
\psi_{p}=2 \cos ^{-1}\left(\frac{1}{x_{0}} \cos \left(\frac{(2 p-1) \pi}{2(N-1)}\right)\right), \quad p=1,2, \ldots, N-1
$$

From (4.47) and the following

$$
z_{p}=e^{j \psi_{p}}, \quad p=1,2, \ldots, N-1,
$$

the symmetric z-transform of the window is constructed in terms of the one-sided transform as follow:

$$
W(z)=z^{-(N-1) / 2} \prod_{p=1}^{N-1}\left(z-z_{p}\right),
$$

The inverse transform of (4.49) will give the Dolph-Chebyshev weights. The following example makes use of this procedure to compute the weights for an array using MATLAB. 
Considering a linear array of 4 elements spaced $\lambda / 2$ apart, we want to achieve optimum mainlobe beam-width with sidelobe level of $20 \mathrm{~dB}$ below the mainlobe level. Using (4.41) results in $R=10$ and considering (4.44) we get:

$$
x_{0}=\cosh \left(\frac{\cosh ^{-1}(10)}{3}\right)=1.540
$$

and the following for the normalized beam pattern:

$$
B(\psi)=\frac{T_{3}\left(1.540 \cos \frac{\psi}{2}\right)}{10}
$$

with these weights:

$$
w=\left[\begin{array}{llll}
1.0000 & 1.7357 & 1.7357 & 1.0000
\end{array}\right]
$$

This results in the following beam pattern in $\theta$-space and $u$-space for a 4-element array:

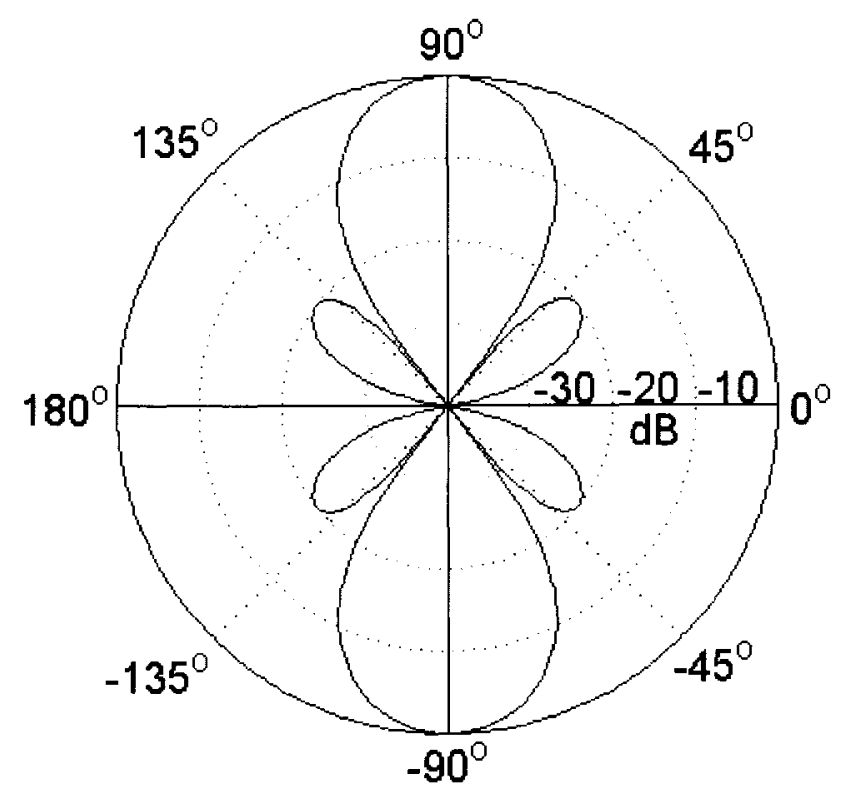

Figure 4.13: Beam Pattern in $\theta$-space using Dolph-Chebyshev Weighting 


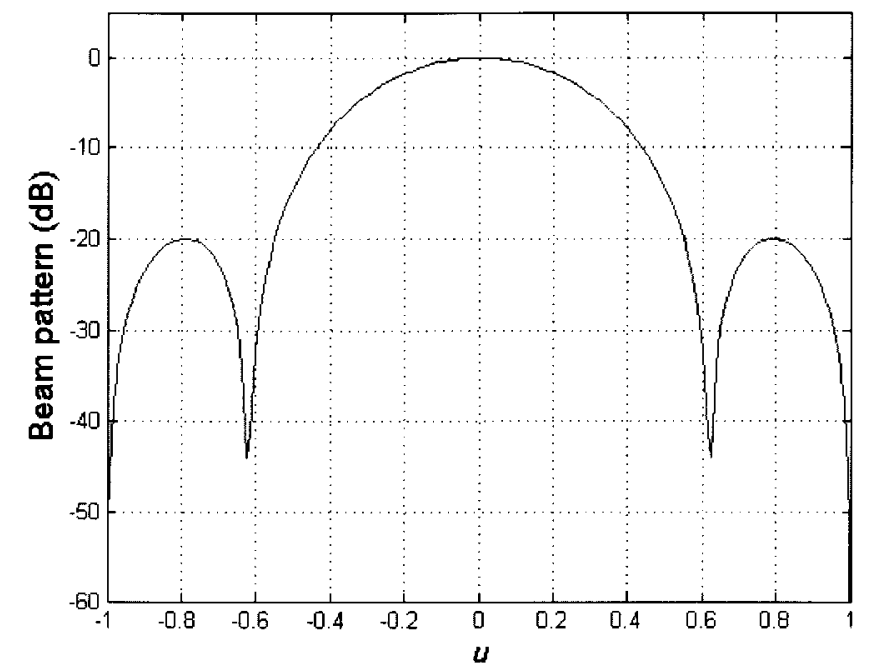

Figure 4.14: Beam Pattern in $u$-space using Dolph-Chebyshev Weighting

Having an 8-element ULA, the following Dolph-Chebyshev weights were calculated in MATLAB:

$$
w=\left[\begin{array}{llllllll}
1.0000 & 1.1386 & 1.5091 & 1.7244 & 1.7244 & 1.5091 & 1.1386 & 1.0000
\end{array}\right]
$$

This results in the following beam pattern in $\theta$-space and $u$-space for an 8 -element array:

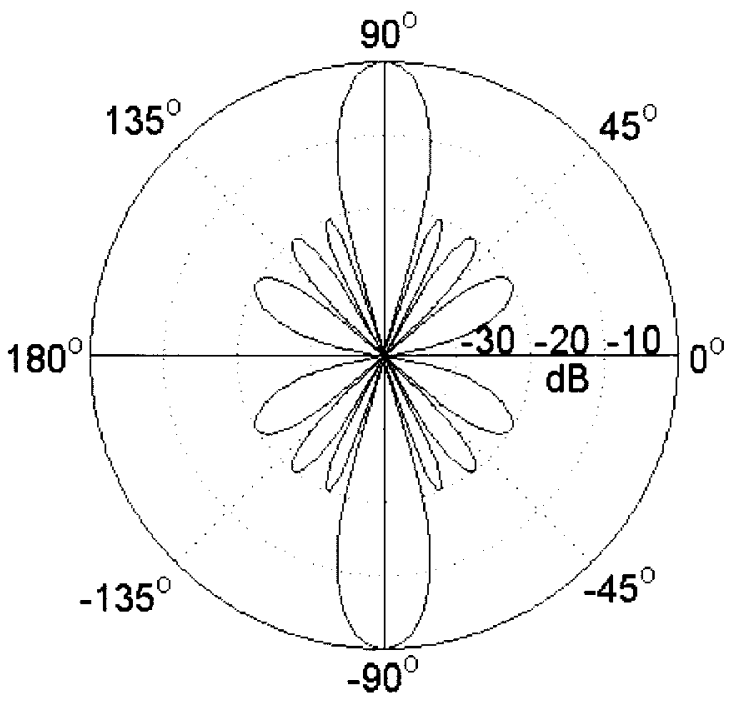

Figure 4.15: 8-element Array Pattern in $\theta$-space using Dolph-Chebyshev Weighting 


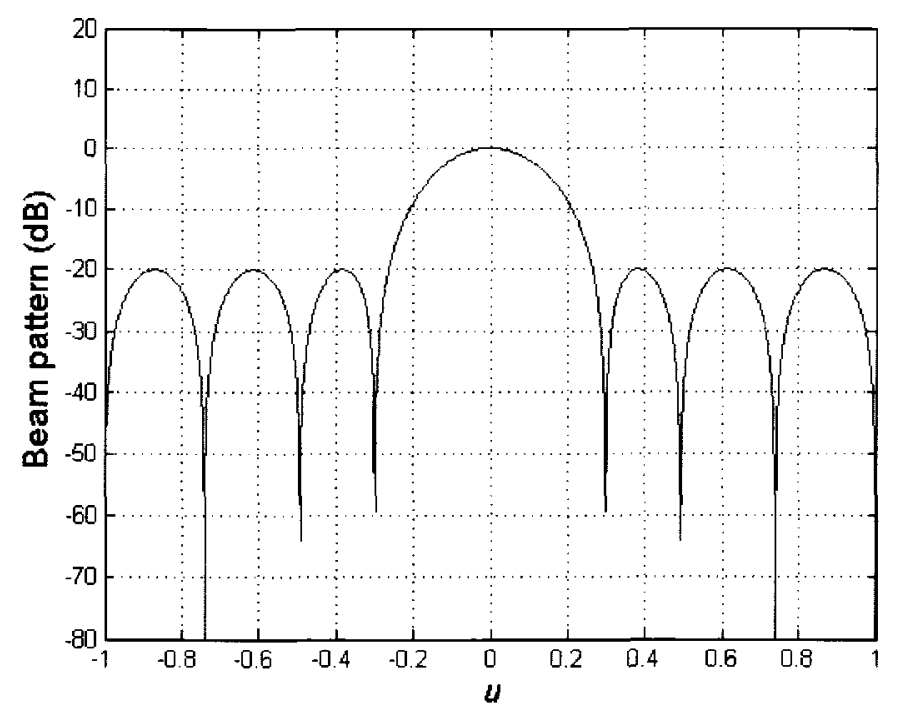

Figure 4.16: 8-element Array Pattern in $u$-space using Dolph-Chebyshev Weighting

It should be noted that the beamwidth (mainlobe width) of the array increases as the side lobe level decreases. Figure 4.17 shows the beam pattern of a 4 -element array with the Dolph-Chebyshev weighting for sidelobe levels of $-10 \mathrm{~dB},-20 \mathrm{~dB}$, and $-30 \mathrm{~dB}$ with respect to the mainlobe level.
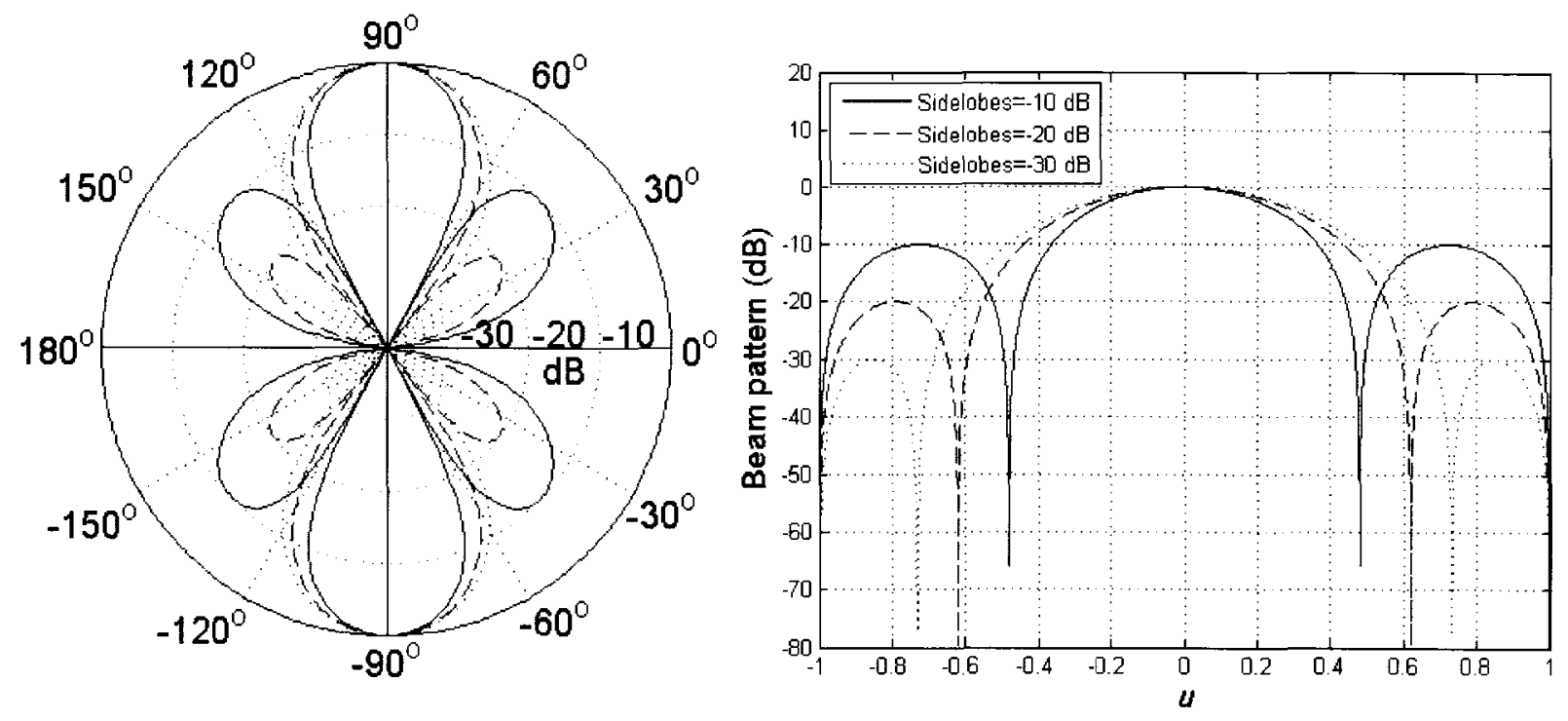

Figure 4.17: Dolph-Chebyshev Beam Pattern for sidelobe $=\left[\begin{array}{lll}-10 d B & -20 d B & -30 d B\end{array}\right]$ 


\subsubsection{Linear Array Null Steering}

The goal, as it was discussed earlier, is to eliminate the effect of jamming $\operatorname{signal}(\mathrm{s})$ that are located at a specific wavenumber $\vec{k}_{\text {jammer }}$. From (4.20), we would like to have the following for the output of the array:

$$
Y\left(\vec{k}_{\text {jammer }}\right)=\vec{w}^{H} \vec{v}_{\bar{k}}\left(\vec{k}_{\text {jammer }}\right)=0 .
$$

or

$$
\sum_{n=0}^{N-1} \omega_{n}^{*} e^{-j\left(n-\frac{N-1}{2}\right) k_{\text {jammer }} d}=0
$$

This is the constraint for having a null in the pattern.

Normally a set of constraints will be defined on the beam pattern at different values of $\vec{k}$. There will also be a constraint on the main response axis of the array in the direction of $\vec{k}_{\text {target }}$. It is called the distortionless constraint, since it allows any plane wave signal coming from $\vec{k}_{\text {target }}$ to pass through the array undistorted. This constraint is defined as follow:

$$
Y\left(\vec{k}_{\text {target }}\right)=\vec{w}^{H} \vec{v}_{\vec{k}}\left(\vec{k}_{\text {target }}\right)=1 .
$$

The first type of constraint is called a zero-order null and it is simply defined by the following $N \times N_{\text {zero }}$ constraint matrix, $\vec{C}_{\text {zero_order }}$ 


$$
\vec{C}_{\text {zero_order }}=\left[\begin{array}{lllll}
\vec{v}_{\vec{k}}\left(\vec{k}_{1}\right) & \vec{v}_{\vec{k}}\left(\vec{k}_{2}\right) & . & . & \vec{v}_{\vec{k}}\left(\vec{k}_{N_{\text {zero }}}\right)
\end{array}\right],
$$

where

$$
\vec{w}^{H} \vec{v}_{\vec{k}}\left(\vec{k}_{i}\right)=0, \quad i=1,2, \ldots, N_{\text {zero }}
$$

defines the set of constraints.

The second type of constraint is called the first-order null, which is the first derivative of the array output (or the beam pattern) with respect to $\vec{k}$ set to zero:

$$
\frac{d}{d k} Y\left(k_{i}\right)=\vec{w}^{H}\left[\frac{d}{d k} \vec{v}_{\vec{k}}\left(k_{i}\right)\right]=0, \quad i \in N_{\text {first }}
$$

where $N_{\text {first }}$ is a subset of $N_{\text {zero }}$ null locations.

This gives a $N \times N_{\text {first }}$ first-order null constraint matrix, $\vec{C}_{\text {first_order }}$ as

$$
\vec{C}_{\text {first_order }}=\left[\begin{array}{l:l:l:l}
\frac{d}{d k} \vec{v}_{\vec{k}}\left(k_{1}\right) & \frac{d}{d k} \vec{v}_{\vec{k}}\left(k_{2}\right) & \cdot \cdot & \frac{d}{d k} \vec{v}_{\vec{k}}\left(k_{N_{\text {frrt }}}\right)
\end{array}\right],
$$

The above matrix is defined for a set of nulls that are chosen to have derivatives set to zero while others do not.

In the same way, the $n$th order constraint matrix can be found by taking the $n$th derivative of the array output. The total constraint matrix $\vec{C}$ becomes

$$
\vec{C}=\left[\begin{array}{l:l:l:l}
\vec{C}_{\text {zero_order }} & \vec{C}_{\text {first_order }} & \vec{C}_{\text {second_order }} & \cdot .
\end{array}\right] .
$$

Note that the columns of $\vec{C}$ are assumed to be linearly independent. 
Defining $\vec{w}_{\text {desired }}^{H}$ to be the desired weighting, the following can be used to find the optimum weighting, $\vec{w}_{\text {optimum }}^{H}$

$$
\vec{w}_{\text {optimum }}^{H}=\vec{w}_{\text {desired }}^{H}-\left(\vec{w}_{\text {desired }}^{H} \vec{C}\left[\vec{C}^{H} \vec{C}\right]^{-1}\right) C^{H}
$$

This means the optimum weight vector is found by subtracting a weighted sum of the constraint vectors from the desired weight vectors. This becomes clear with the following example, where we impose a constraint on an 8-element linear array with Dolph-Chebyshev weighting. The null is set to be at $\theta=30^{\circ}$ or $\mathrm{u}=\cos \theta=0.866$. The first set of figures show the beam pattern without imposing the null, followed by zero, first, and second order null constraint.
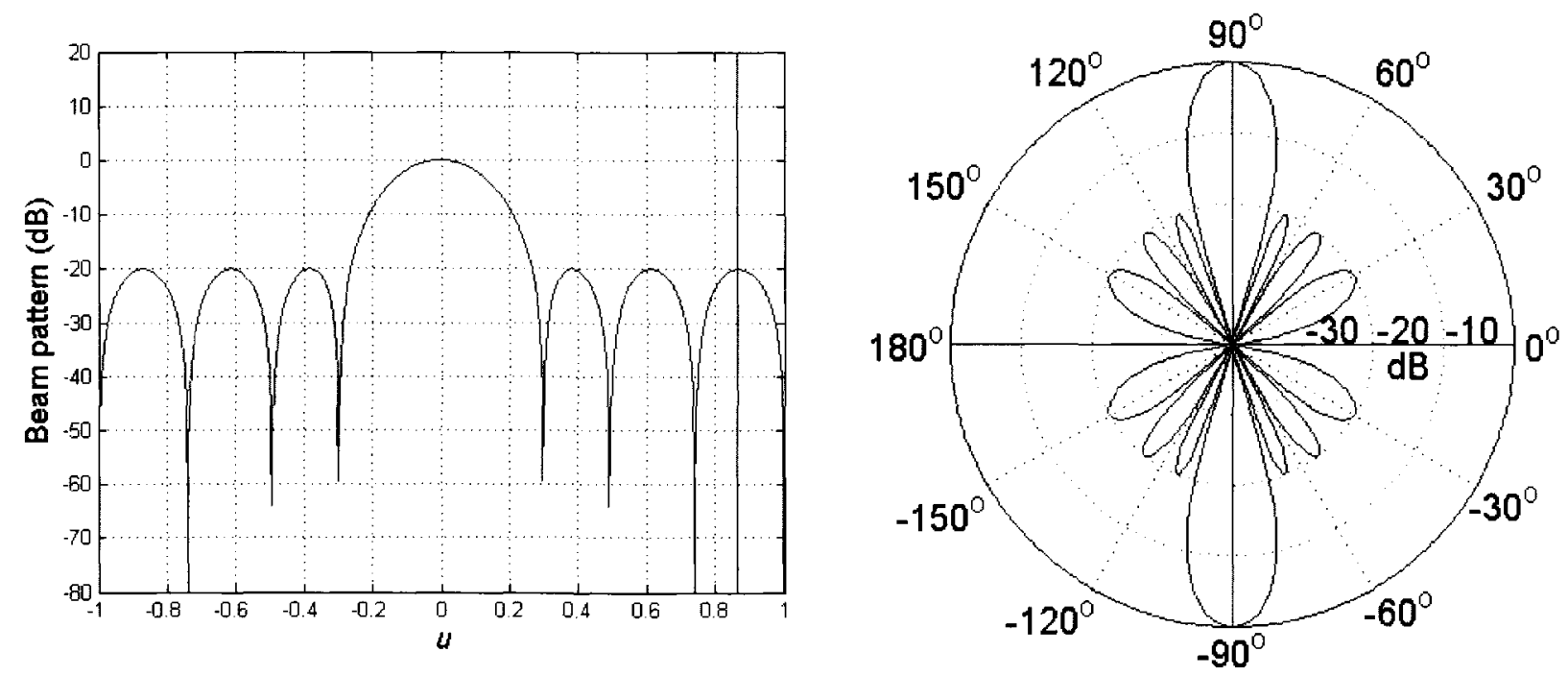

Figure 4.18: 8-element Array Beam Pattern without Imposing Null 

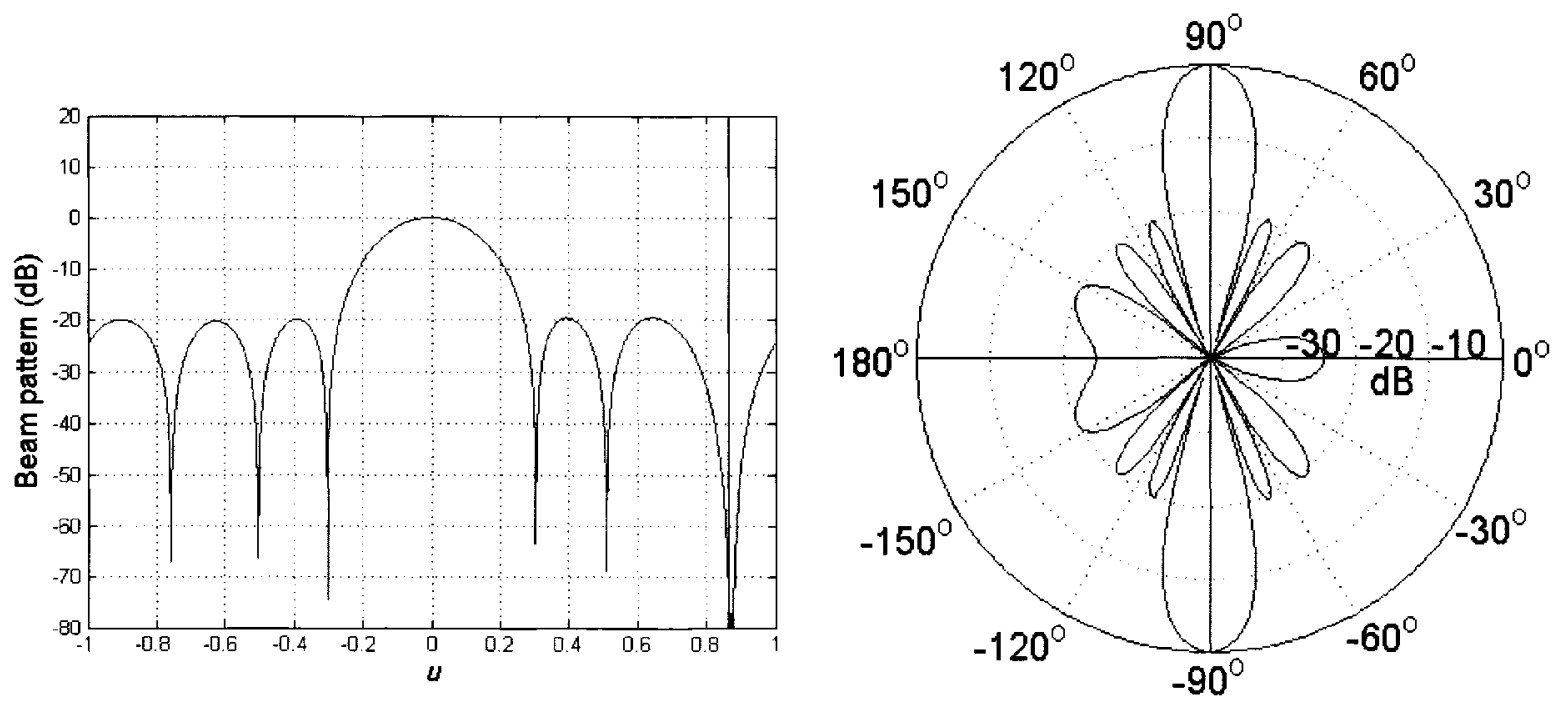

Figure 4.19: 8 -element Array Beam Pattern with Zero-Order Null at $u=0.866$ or $\theta=30^{\circ}$
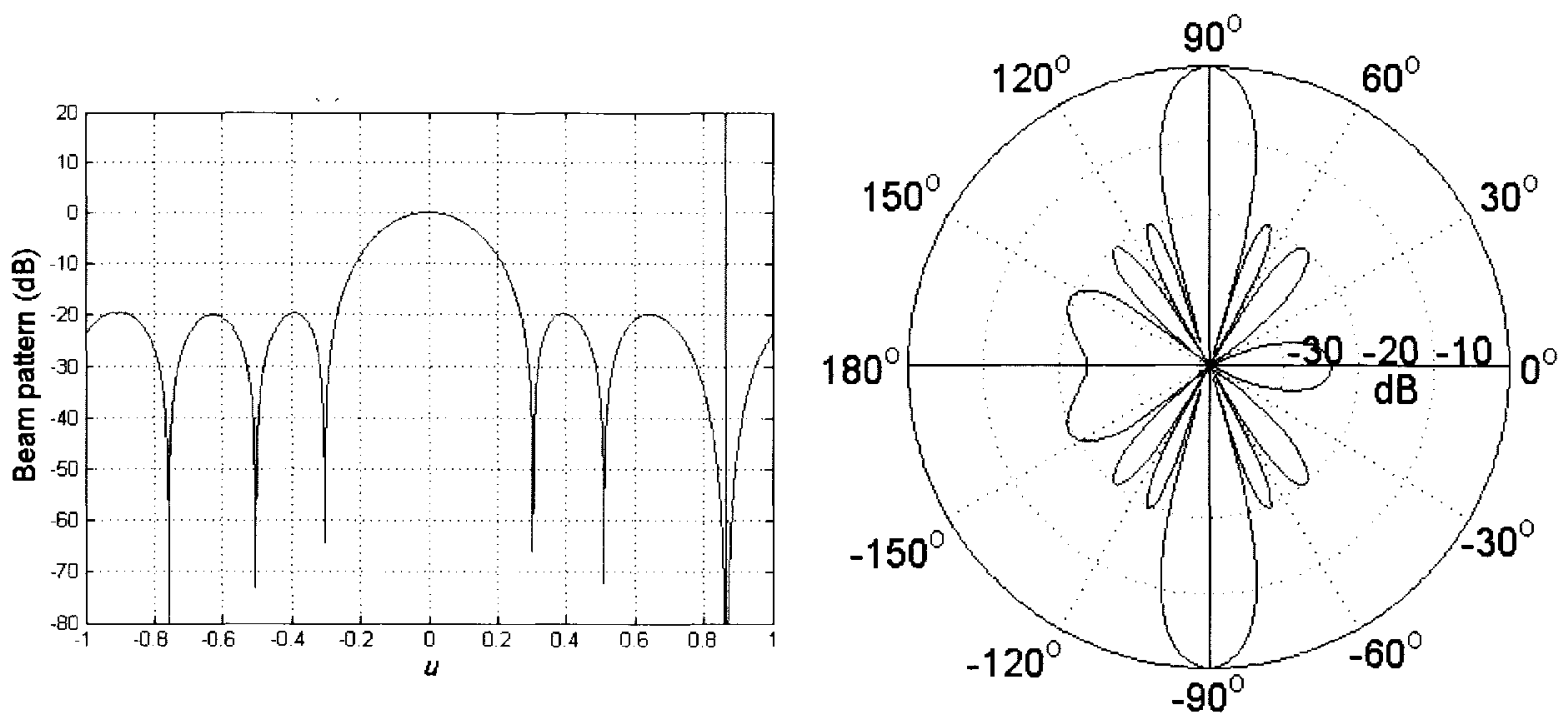

Figure 4.20: 8-element Array Beam Pattern with First-Order Null at $u=0.866$ or $\theta=30^{\circ}$ 

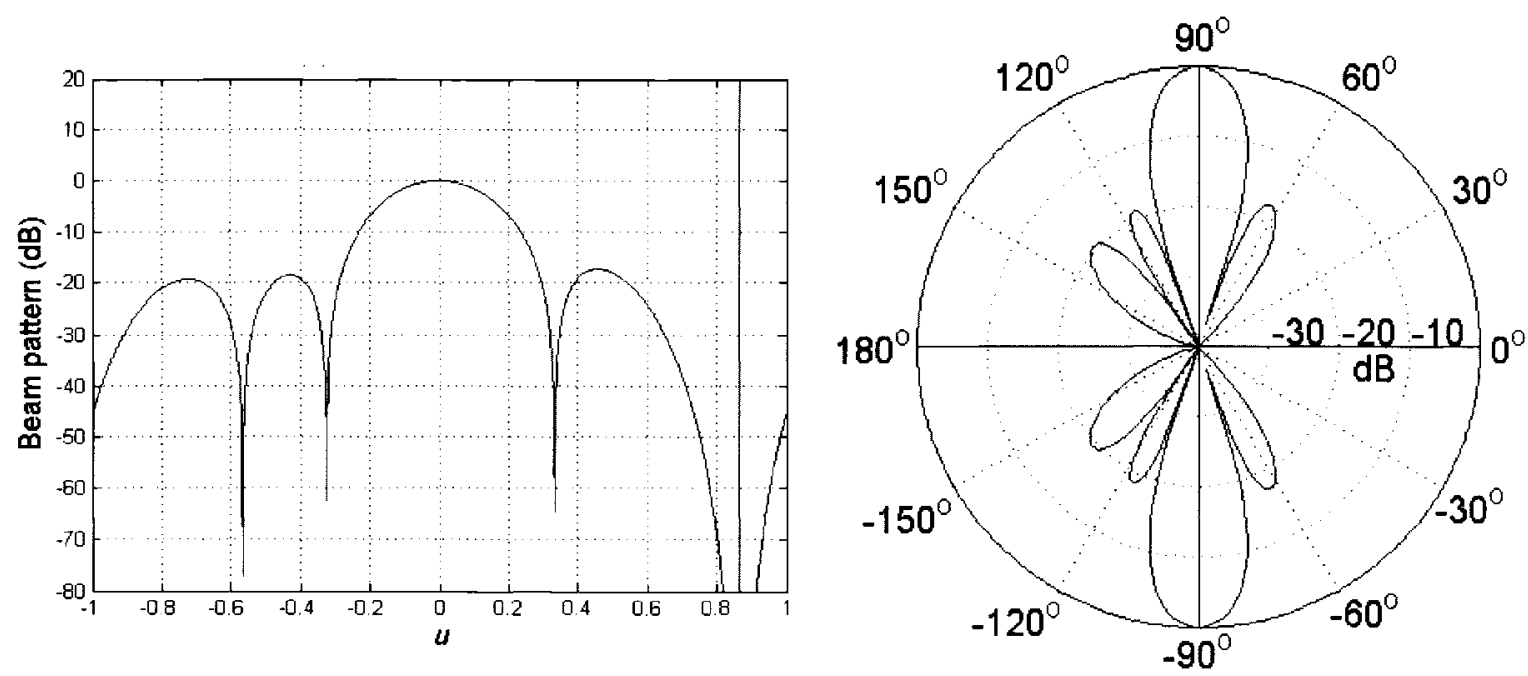

Figure 4.21: 8-element Array Beam Pattern with 2nd-Order Null at $u=0.866$ or $\theta=30^{\circ}$

The following figures show the effects of a zero, first, and second-order null on a 4-element linear array. The results can be compared to the results for an 8-element linear array shown in previous figures. The effect of nulling on the beam pattern becomes more severe as the order of nulling increases. This effect is really extraordinary when using the second-order nulling on a 4-element linear array as can be seen in Figure 4.24.
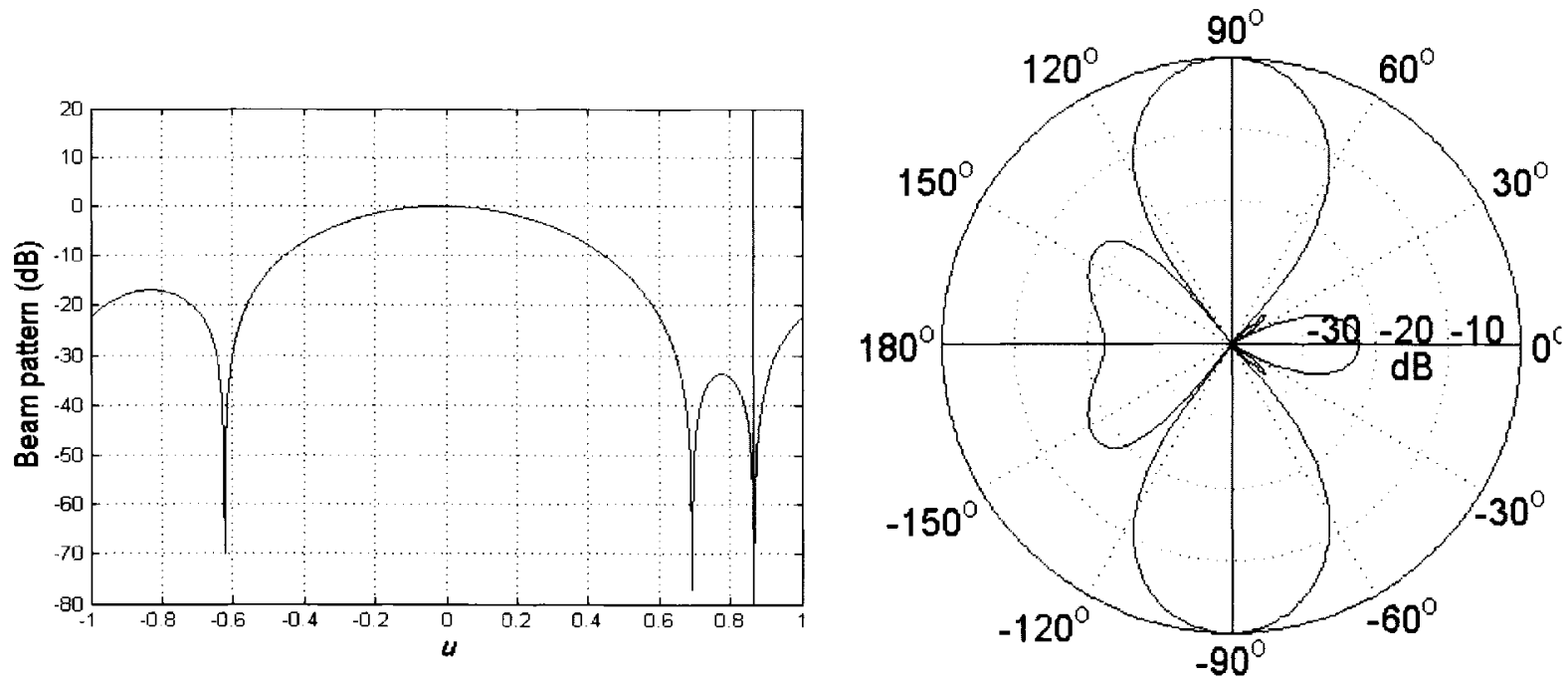

Figure 4.22: 4-Element Array Beam Pattern with Zero-Order Null at $u=0.866$ or $\theta=30^{\circ}$ 

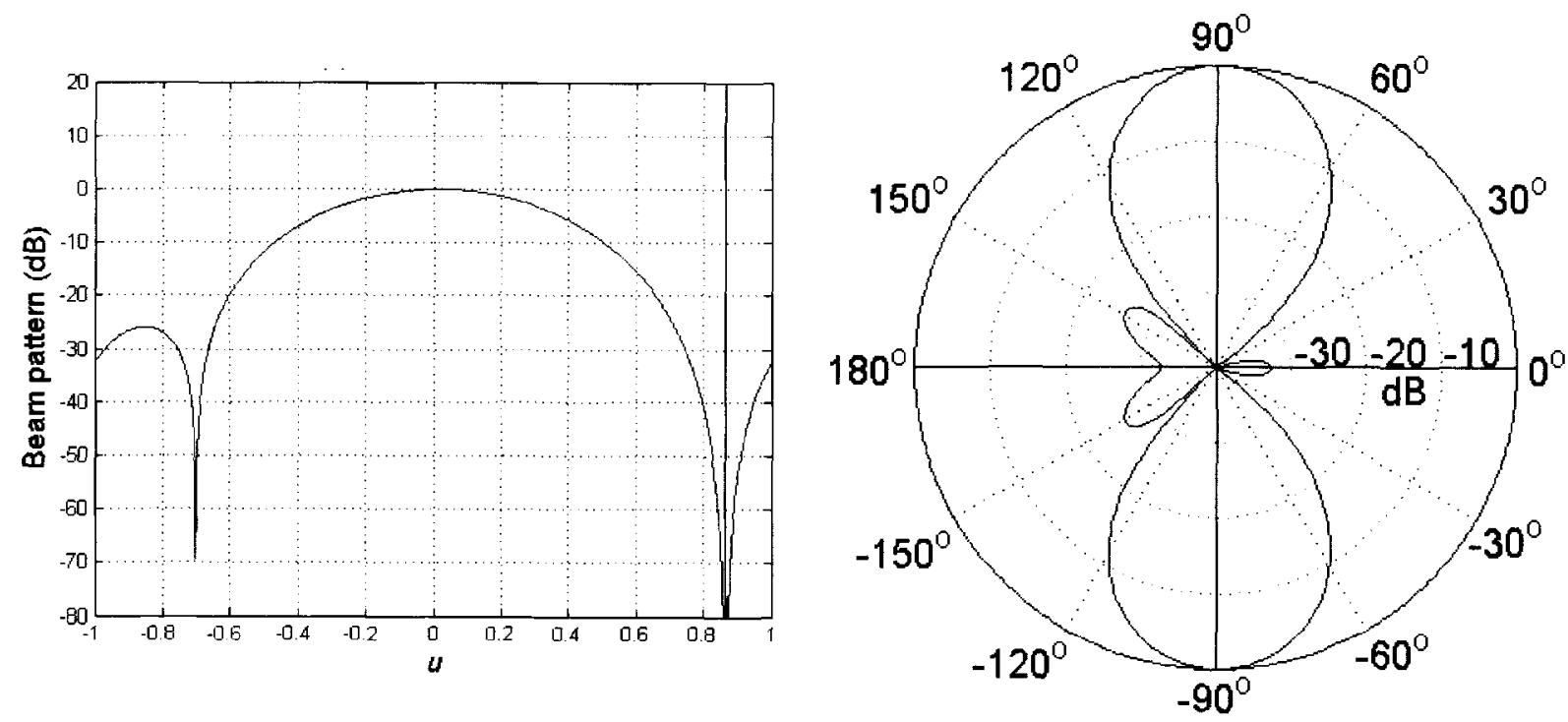

Figure 4.23: 4-Element Array Beam Pattern with First-Order Null at $u=0.866$ or $\theta=30^{\circ}$
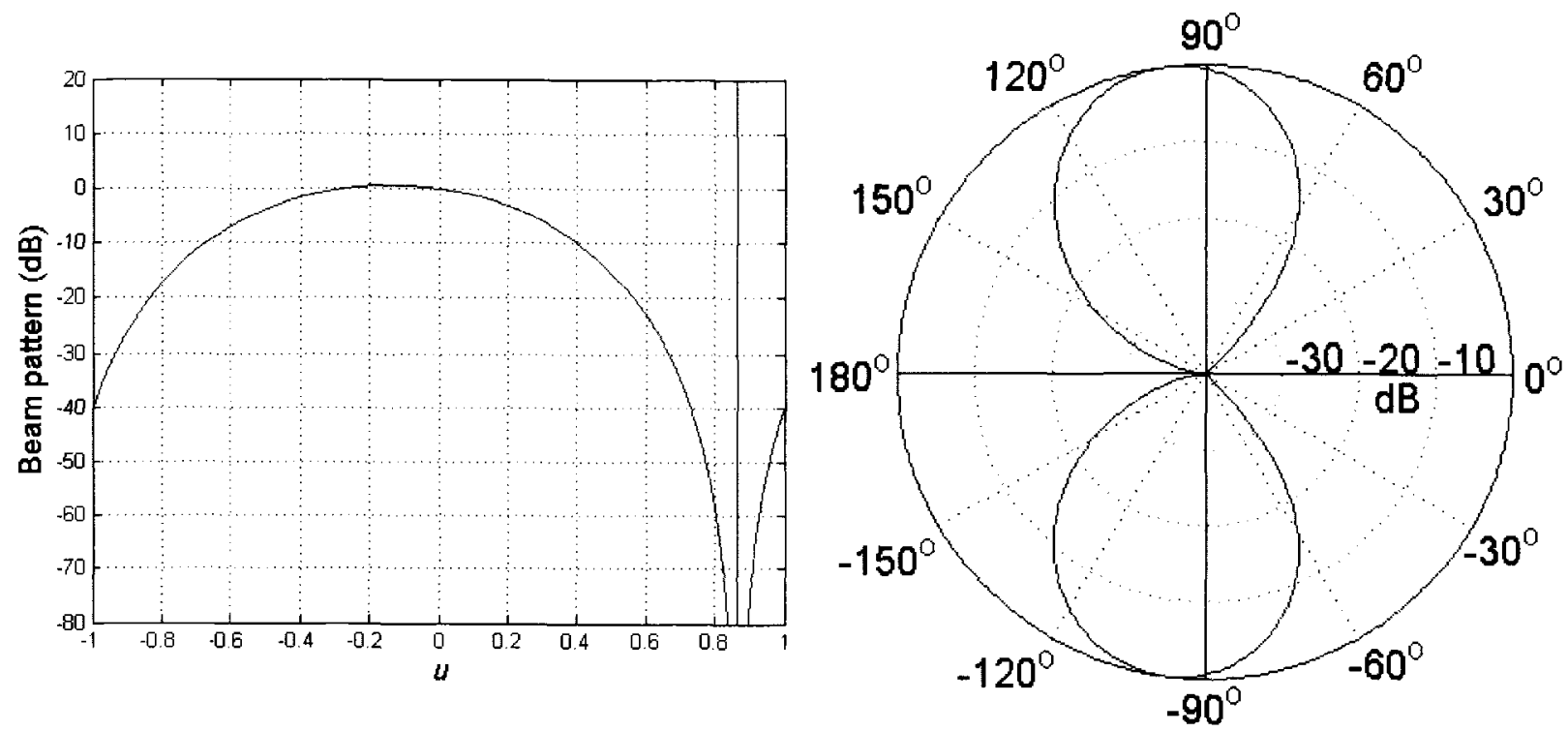

Figure 4.24: 4-Element Array Beam Pattern with Second-Order Null at $u=0.866$ or $\theta=30^{\circ}$ 
The above null steering method can be easily expanded to handle several numbers of interference directions. The figures below shows an 8-element Dolph-Chebyshev array beam pattern with three zero-order nulls imposed on it at $u=\left[\begin{array}{lll}0.866 & 0.500 & -0.7071\end{array}\right]$ or $\theta=\left[\begin{array}{lll}30^{\circ} & 60^{\circ} & -45^{\circ}\end{array}\right]$.

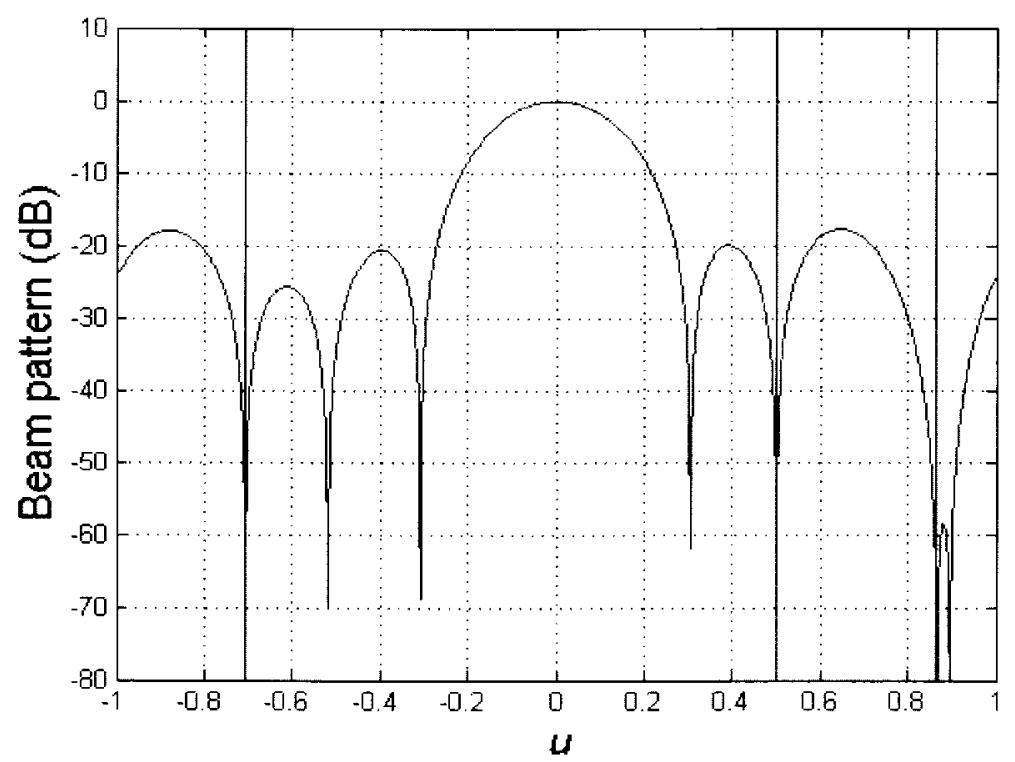

Figure 4.25: 8-Element Array with 3 Zero-Order Null at $u=\left[\begin{array}{lll}0.866 & 0.500 & -0.7071\end{array}\right]$

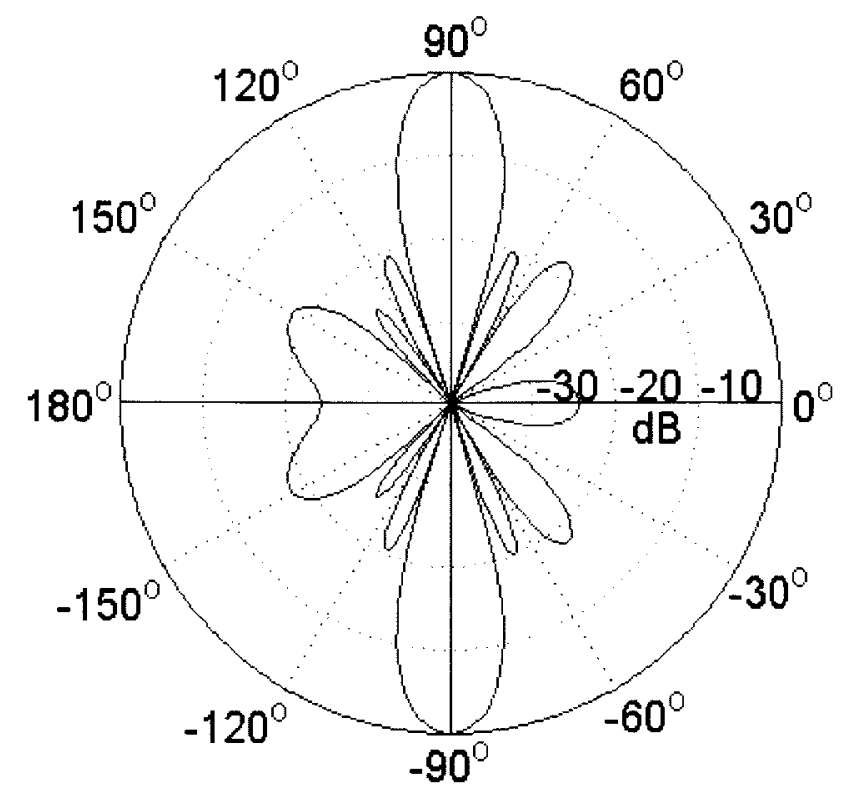

Figure 4.26: 8-Element Array with 3 Zero-Order Null at $\theta=\left[\begin{array}{lll}30^{\circ} & 60^{\circ} & -45^{\circ}\end{array}\right]$ 
For the purpose of comparison, the following figures show the 4-element array beam pattern with three zero-order null at the same direction as the 8-element array (shown in previous two figures).

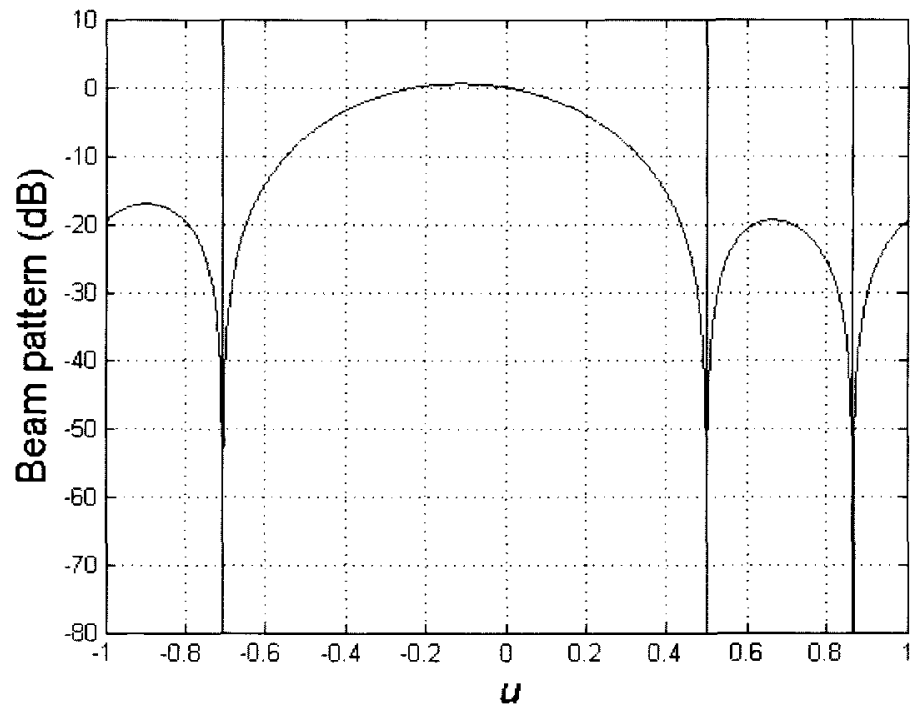

Figure 4.27: 4-Element Array with 3 Zero-Order Null at $u=\left[\begin{array}{lll}0.866 & 0.500 & -0.7071\end{array}\right]$

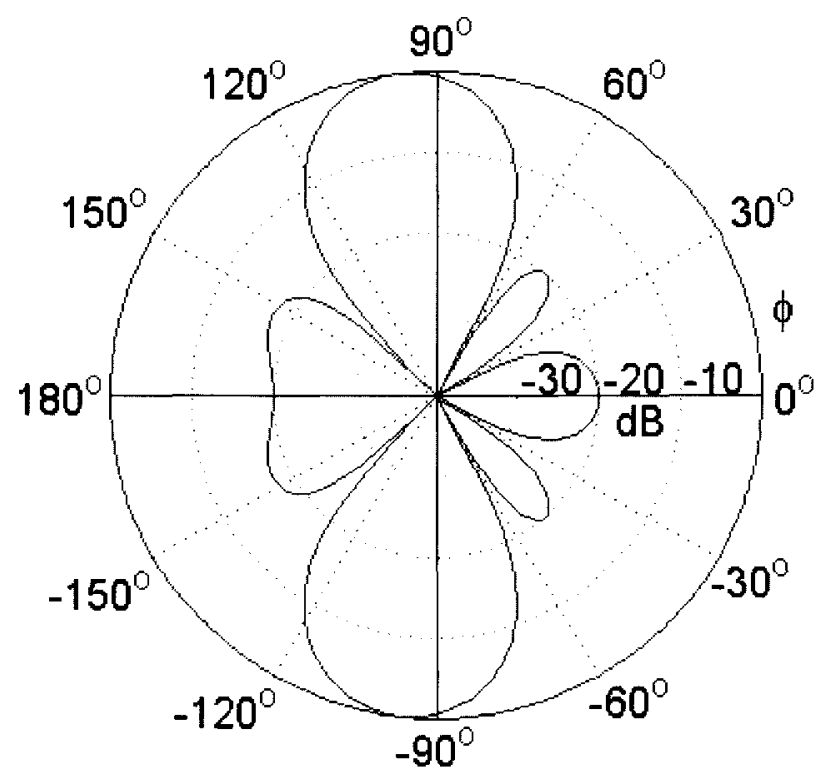

Figure 4.28: 8-Element Array with 3 Zero-Order Null at $\theta=\left[\begin{array}{lll}30^{\circ} & 60^{\circ} & -45^{\circ}\end{array}\right]$ 


\subsection{Planar Arrays Beam Pattern}

\subsubsection{Planar Array Overview}

The most used grid in designing planar arrays is a rectangular grid with uniform spacing. The geometry of these types of array is illustrated in Figure 4.29.

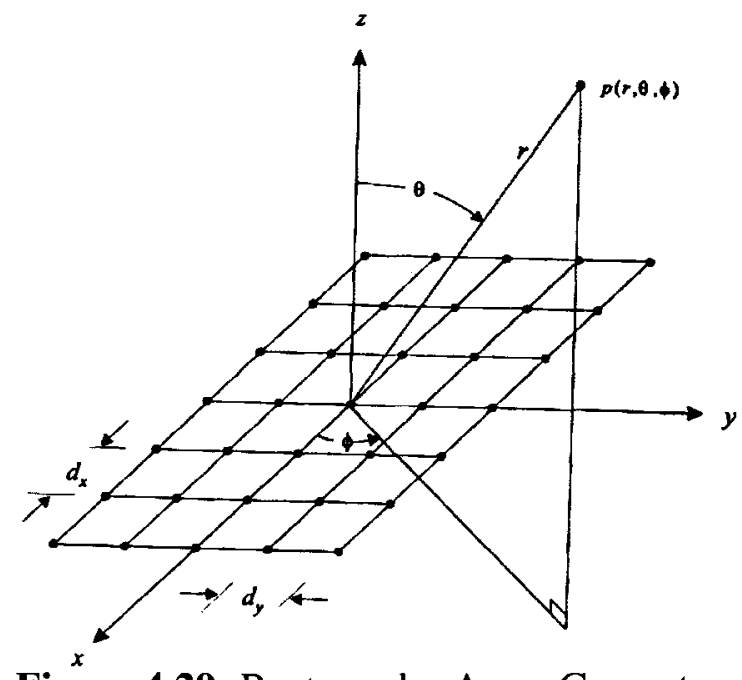

Figure 4.29: Rectangular Array Geometry

In this thesis we consider analysis of planar arrays whose elements are located on the $x y$-plane. Many concepts, which are developed for linear arrays can be further expanded to be suitable for planar arrays. Following the output of a ULA (4.20) and (4.22), the beam pattern of a $N \times M$ uniform rectangular array in $\psi$-space can be found as

$$
B\left(\psi_{x}, \psi_{y}\right)=e^{-j\left(\frac{N-1}{2} \psi_{x}\right)} e^{-j\left(\frac{M-1}{2} \psi_{y}\right)} \sum_{n=0}^{N-1} \sum_{m=0}^{M-1} w_{n m}^{*} e^{j\left(n \psi_{x}\right)} e^{j\left(m \psi_{y}\right)}
$$

where

$$
\begin{aligned}
& \psi_{x}=\frac{2 \pi}{\lambda} d_{x} \sin \theta \cos \phi, \\
& \psi_{y}=\frac{2 \pi}{\lambda} d_{y} \sin \theta \sin \phi,
\end{aligned}
$$


Considering the case of standard uniform rectangular array (SURA) where

$$
d_{x}=d_{y}=\frac{\lambda}{2}
$$

(4.60) becomes

$$
\begin{aligned}
& \psi_{x}=\pi \sin \theta \cos \phi=\pi u_{x}, \\
& \psi_{y}=\pi \sin \theta \sin \phi=\pi u_{y}
\end{aligned}
$$

This results in the following beam pattern in $u$-space:

$$
B\left(u_{x}, u_{y}\right)=e^{-j\left(\frac{N-1}{2} \pi u_{x}\right)} e^{-j\left(\frac{M-1}{2} \pi u_{y}\right)} \sum_{n=0}^{N-1} \sum_{m=0}^{M-1} w_{n m}^{*} e^{j\left(n \pi u_{x}\right)} e^{j\left(m \pi u_{y}\right)},
$$

The beam pattern in (4.59), in the case of uniform weighting, can be seen as the product of two separate factors as

$$
\begin{aligned}
B\left(\psi_{x}, \psi_{y}\right) & =B_{x}\left(\psi_{x}\right) \cdot B_{y}\left(\psi_{y}\right) \\
& =\left(\frac{1}{N} \frac{\sin \left(\frac{N}{2} \psi_{x}\right)}{\sin \left(\frac{\psi_{x}}{2}\right)}\right) \cdot\left(\frac{1}{M} \frac{\sin \left(\frac{M}{2} \psi_{y}\right)}{\sin \left(\frac{\psi_{y}}{2}\right)}\right) .
\end{aligned}
$$

The following figures show the amplitude of the beam pattern found (4.63) or (4.64) in $u$-space, for the case of uniform weighting. In the first figure we consider the case where $M=N$, which is the most common configuration in designing planar arrays. 

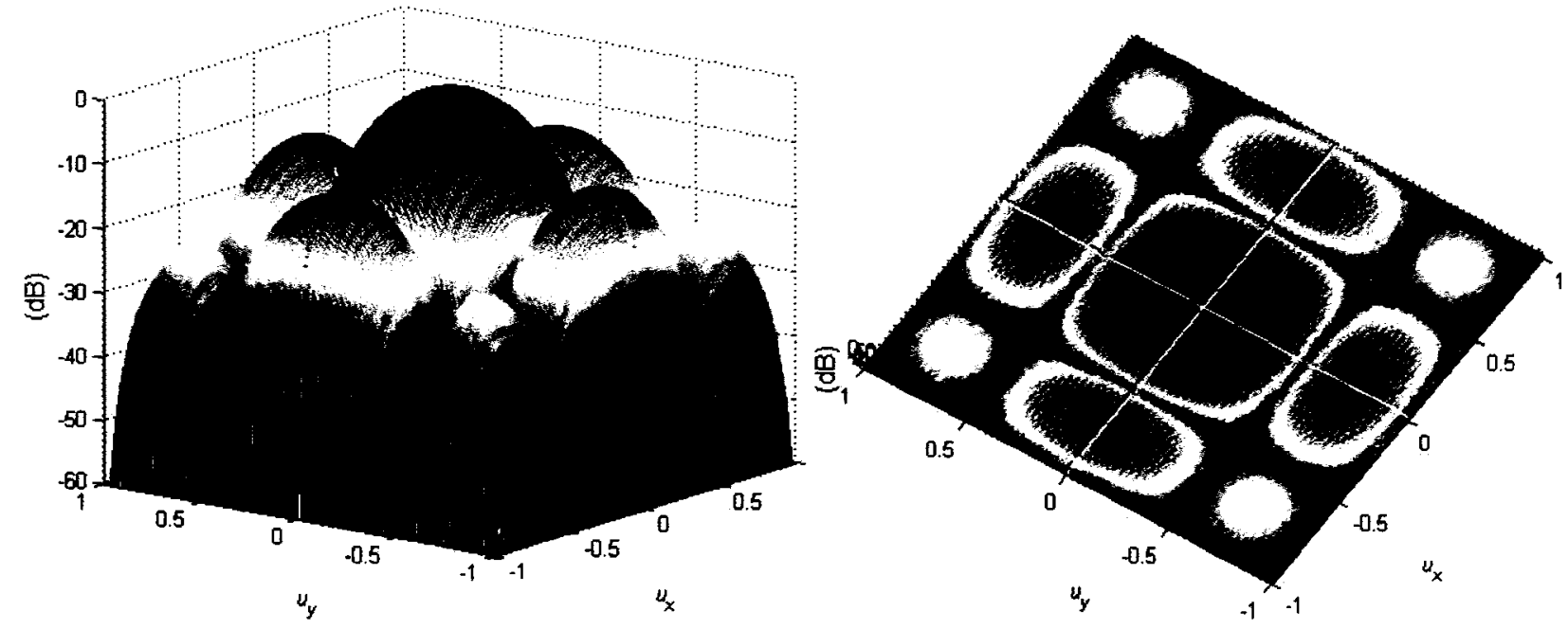

Figure 4.30: Two Views of 4x4 Standard Rectangular Array's Beam Pattern with

Uniform Weighting

Figure 4.31 shows the beam pattern for a rectangular array where $N=8$ and $M=4$ with uniform weighting.

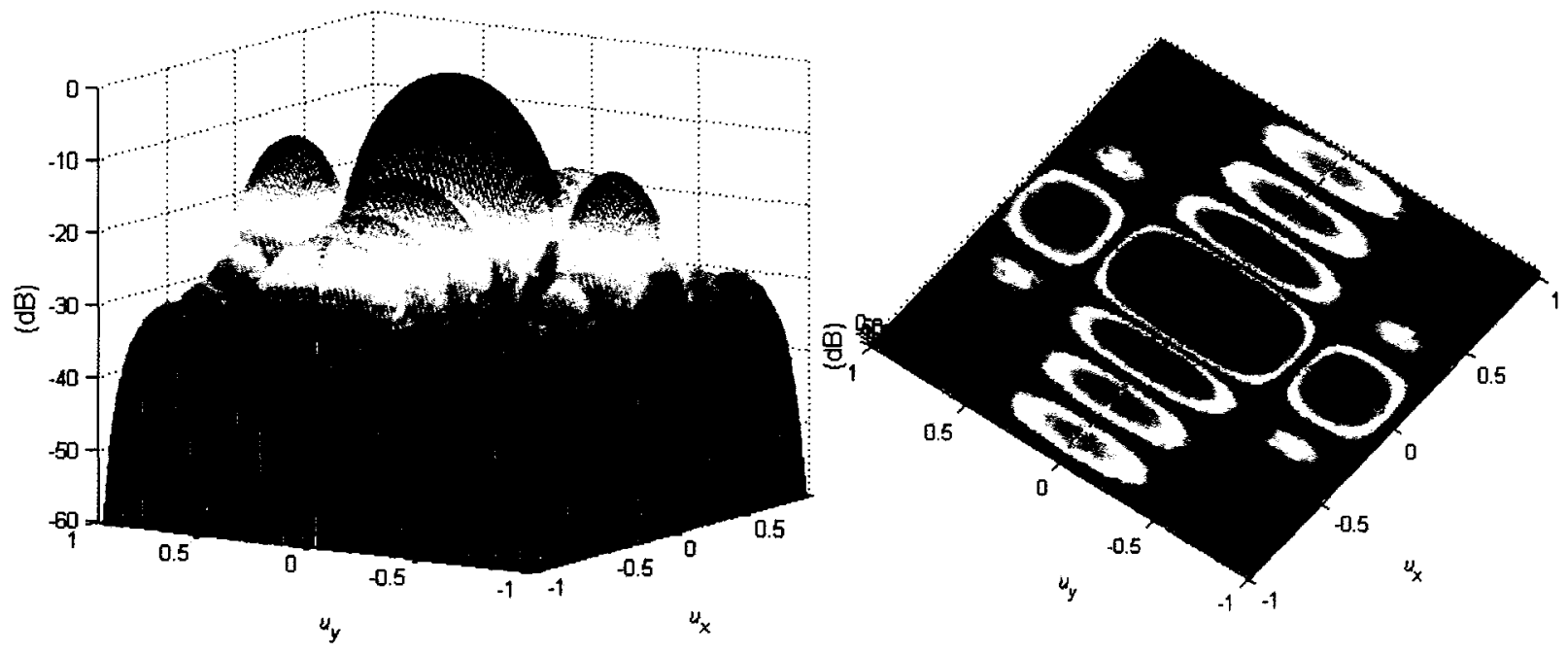

Figure 4.31: Two Views of $8 \times 4$ Rectangular Array's Beam Pattern with Uniform Weighting 


\subsubsection{Dolph-Chebyshev Weighting for SURAs}

Now back to the case of Dolph-Chebyshev weighting, the goal is to find and apply the weights to achieve the optimum beam pattern of the $N \times N$ planar array. The array beam pattern for a linear array with Dolph-Chebyshev weighting, found in (4.45), can be modified to suit the case of a rectangular array as follow

$$
B\left(\psi_{x}, \psi_{y}\right)=\frac{T_{N-1}\left(x_{0} \cos \frac{\psi_{x}}{2} \cos \frac{\psi_{y}}{2}\right)}{R},
$$

where $x_{0}$ can be found using the same equation as for the case of a linear array (4.44), $\psi_{x}$ and $\psi_{y}$ are defined by (4.62).

Now after sampling the beam pattern, we can define the following:

$$
B\left(l_{1}, l_{2}\right)=B_{\psi}\left(\psi_{x}, \psi_{y}\right) e^{-j \frac{N-1}{2}\left(\psi_{x l_{1}}+\psi_{y_{2}}\right)},
$$

where

$$
\begin{aligned}
& \psi_{x l_{1}}=\frac{2 \pi}{N}\left(l_{1}-\frac{N-1}{2}\right), \\
& \psi_{x l_{2}}=\frac{2 \pi}{N}\left(l_{2}-\frac{N-1}{2}\right) .
\end{aligned}
$$

and

$$
\begin{aligned}
& l_{1}=0,1, \ldots, N-1, \\
& l_{2}=0,1, \ldots, N-1
\end{aligned}
$$

Equation (4.66) can be written as

$$
B\left(l_{1}, l_{2}\right)=\sum_{n=0}^{N-1} \sum_{m=0}^{N-1} B(n, m) e^{-j \frac{2 \pi}{N}\left(l_{1} n+l_{2} m\right)},
$$


where

$$
B(n, m)=w(n, m) e^{j \frac{N-1}{N}(n \pi+m \pi)},
$$

This means that if $B(n, m)$ is found from (4.70), the desired weights $w(n, m)$, can be calculated. As it is obvious that (4.70) is a 2-D DFT, so by taking the IDFT we can get $B(n, m)$ as follow:

$$
B(n, m)=\frac{1}{N^{2}} \sum_{l_{2}=0}^{N-1} \sum_{l_{2}=0}^{N-1} B\left(l_{1}, l_{2}\right) e^{j \frac{2 \pi}{N}\left(l_{1} n+l_{2} m\right)},
$$

Having found $B(n, m)$ and $B\left(l_{1}, l_{2}\right)$, the weights can be obtained. This procedure was implemented in MATLAB to find the weights, and is used for plotting the beam pattern of an $4 \times 4$ array with a sidelobe level of $-20 \mathrm{~dB}$ with respect to the mainlobe level:
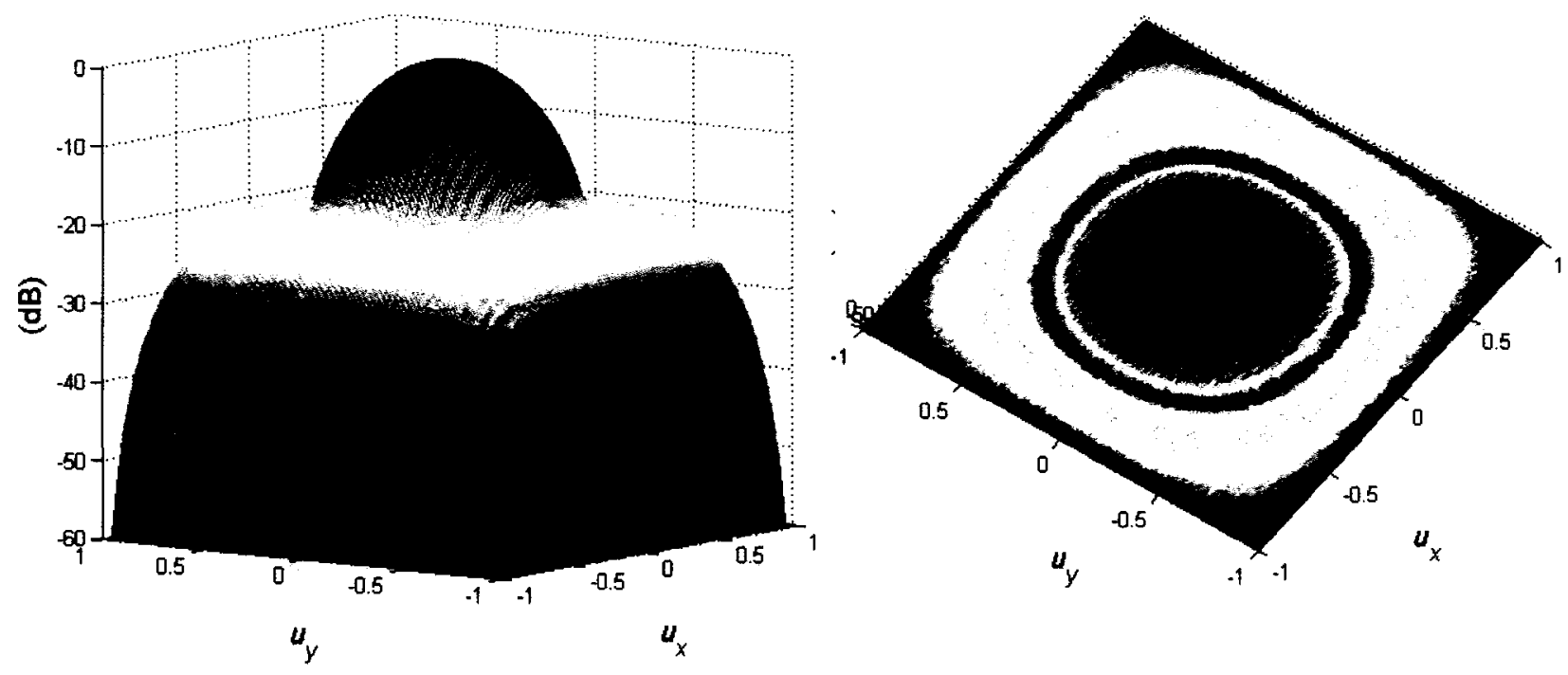

Figure 4.32: Beam Pattern of a $4 \times 4$ Standard Rectangular Array with Dolph-Chebyshev Weighting 


\begin{tabular}{|c|c|c|c|c|}
\hline$w(n, m)$ & $m=1$ & $m=2$ & $m=3$ & $m=4$ \\
\hline$n=1$ & 0.2536 & 0.7609 & 0.7609 & 0.2536 \\
\hline$n=2$ & 0.7609 & 1.0000 & 1.0000 & 0.7609 \\
\hline$n=3$ & 0.7609 & 1.0000 & 1.0000 & 0.7609 \\
\hline$n=4$ & 0.2536 & 0.7609 & 0.7609 & 0.2536 \\
\hline
\end{tabular}

Table 4.2: Dolph-Chebyshev Weighting for a $4 \times 4$ SRA

Again for comparison purposes the beam pattern for an $8 \times 8$ standard rectangular array with Dolph-Chebyshev weighting is shown in Figure 4.33.
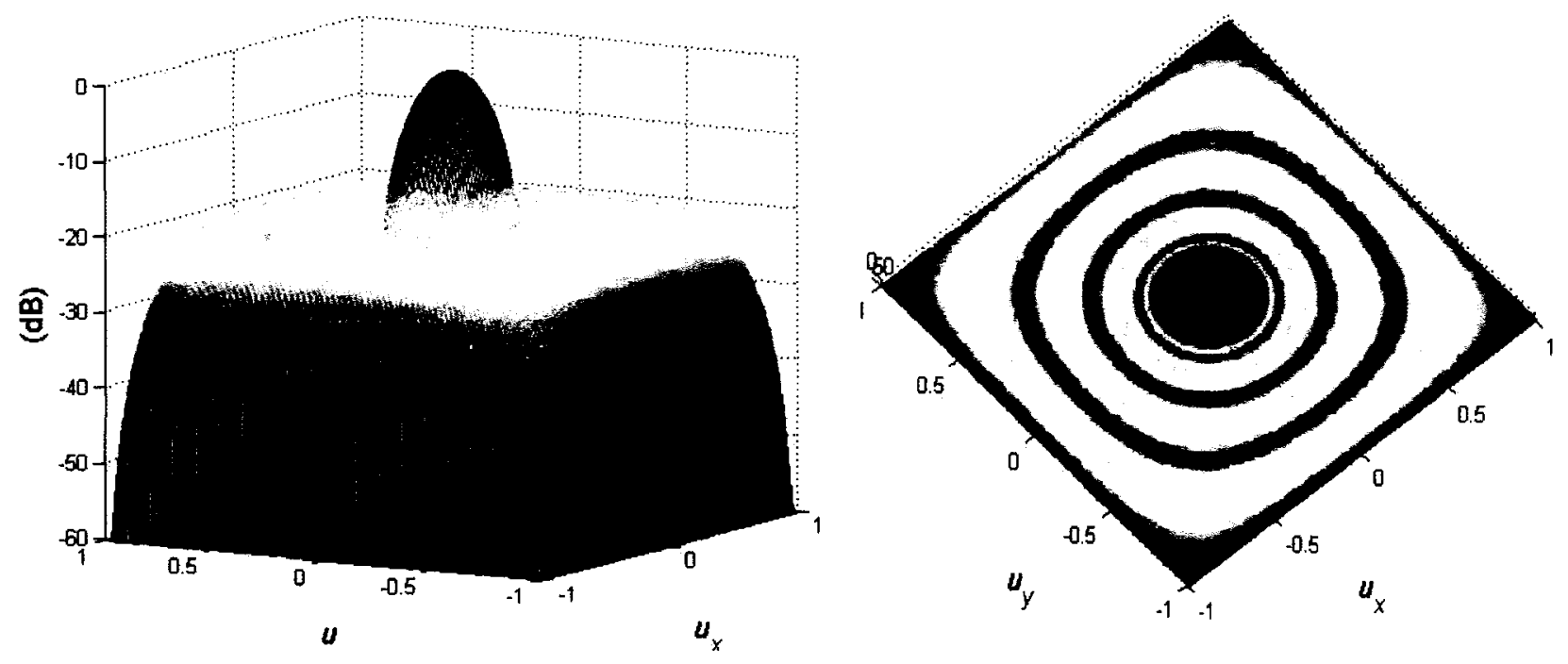

Figure 4.33: Beam Pattern of a $8 \times 8$ Standard Rectangular Array with Dolph-Chebyshev

Weighting 


\subsubsection{Null Steering Using Planar Arrays}

The array manifold vector for a linear array was defined in (4.24). In the case of a rectangular planar array an array manifold matrix will be used instead, where the array manifold vector corresponding to its $m$ th line of elements in the $y$-direction (or its $m$ th column) is defined as

$$
\vec{v}_{m}(\psi)=\left[\begin{array}{c}
e^{j m \psi_{y}} \\
e^{j\left(\psi_{x}+m \psi_{y}\right)} \\
\cdot \\
\cdot \\
e^{j\left((N-1) \psi_{x}+m \psi_{v}\right)}
\end{array}\right]
$$

This results in the following $N \times M$ manifold matrix:

$$
\vec{V}_{\psi}(\psi)=\left[\begin{array}{l:l:l:l|l}
\vec{v}_{0}(\psi) & \vec{v}_{1}(\psi) & \vec{v}_{M-1}(\psi)
\end{array}\right]
$$

The same analogy can be considered for the weight matrix. The weight vector of the $m$ th line of elements is

$$
\vec{w}_{m}=\left[\begin{array}{c}
w_{0, m} \\
w_{1, m} \\
\cdot \\
\cdot \\
\cdot \\
w_{N-1, m}
\end{array}\right],
$$

and the weight matrix is

$$
\vec{W}=\left[\begin{array}{llllll}
\vec{w}_{0} & \vec{w}_{1} & \cdot & \cdot & \vec{w}_{M-1}
\end{array}\right]
$$


The null constraint matrix can be defined the same way the linear array null constraint vector (4.53) was found.

$$
\vec{C}_{\text {zero_order }}=\left[\begin{array}{l:l:l:l}
\vec{V}_{\psi}\left(\psi_{1}\right) & \vec{V}_{\psi}\left(\psi_{2}\right) & \cdot . & \vec{V}_{\psi}\left(\psi_{N_{\text {zero }}}\right)
\end{array}\right],
$$

Where $N_{\text {zero }}$ is the number of null constraints.

The optimum weighting found in (4.58) can be directly applied here:

$$
\vec{w}_{\text {optimum }}^{H}=\vec{w}_{\text {desired }}^{H}-\left[\vec{w}_{\text {desired }}^{H} \vec{C}_{\text {zero_order }}\left[\vec{C}^{H}{ }_{\text {zero_order }} \vec{C}_{\text {zero_order }}\right]^{-1}\right] C^{H}{ }_{\text {zero_order }},
$$

This results in the following optimum beam pattern:

$$
\begin{aligned}
B_{\text {optimum }}(\psi) & =B_{\text {desired }}(\psi) \\
& -\left(\left[\vec{w}_{\text {desired }}^{H} \vec{C}_{\text {zero_order }}\left[\vec{C}^{H}{ }_{\text {zero_order }} \vec{C}_{\text {zero_order }}\right]^{-1}\right] C^{H}{ }_{\text {zero_order }}\right) \vec{V}_{\psi}^{T}(\psi),
\end{aligned}
$$

The above procedure was also developed in MATLAB to find the optimum beam pattern in the presence of null(s). Again first we consider the case of a $4 \times 4$ standard rectangular array with Dolph-Chebyshev weighting $(-20 \mathrm{~dB}$ sidelobe level) and then we impose only one null constraint on the beam pattern. Then the case for the $8 \times 8$ array and multiple null locations are developed and are shown in the figures following the case for $4 \times 4$ array. Note the following:

$$
\begin{aligned}
& u_{x}=\sin \theta \cos \phi \\
& u_{y}=\sin \theta \sin \phi
\end{aligned}
$$




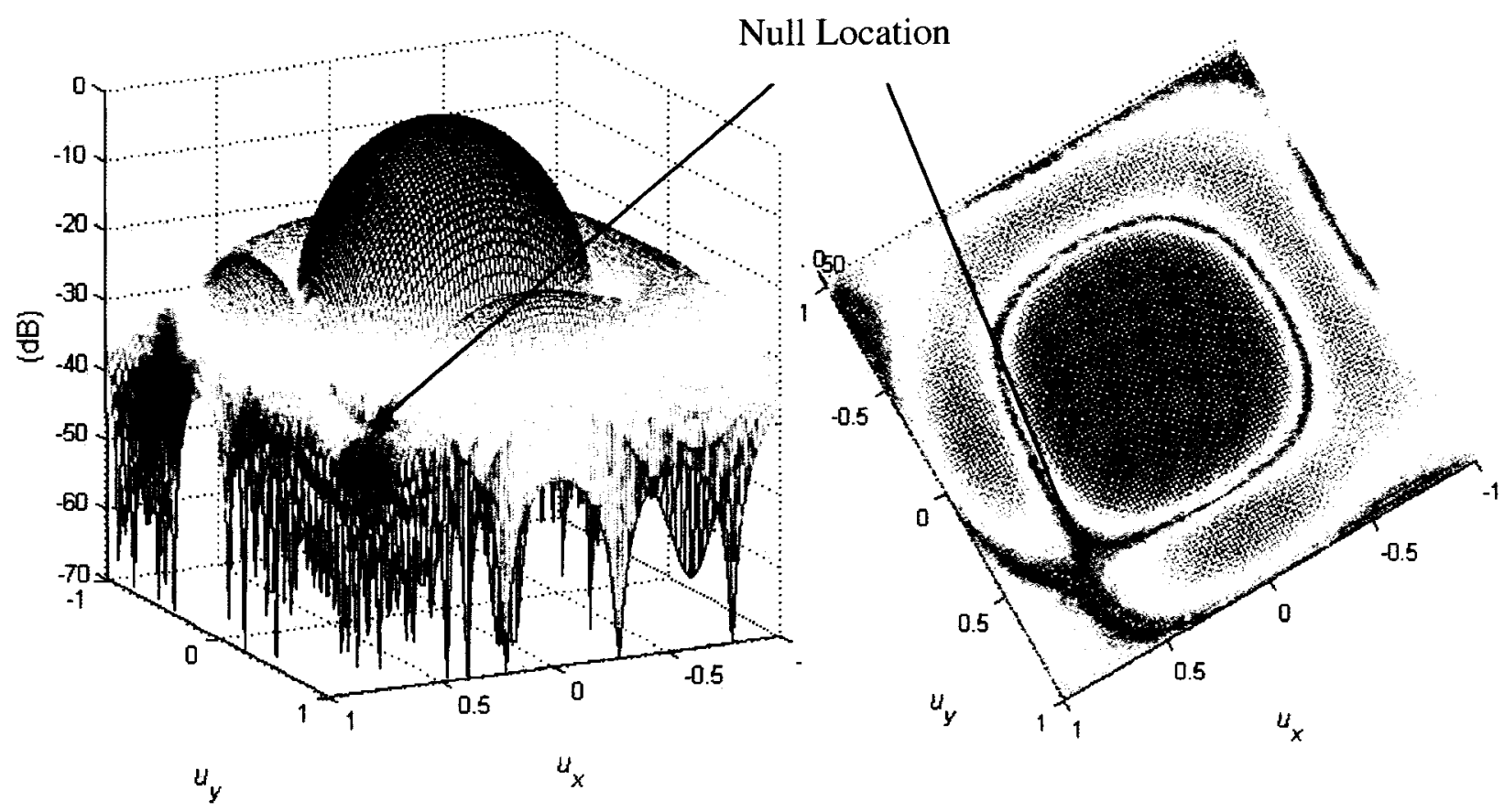

Figure 4.34: Different Views of $4 \times 4$ Array's Beam Pattern with null at

$$
\theta=70^{\circ}, \phi=40^{\circ} \text { or } u_{x}=0.72, u_{y}=0.60
$$

Figure 4.35 shows the effect of putting a null over the mainlobe. Although the level of sidelobes rises as expected, the null is effectively placed in the desired location.

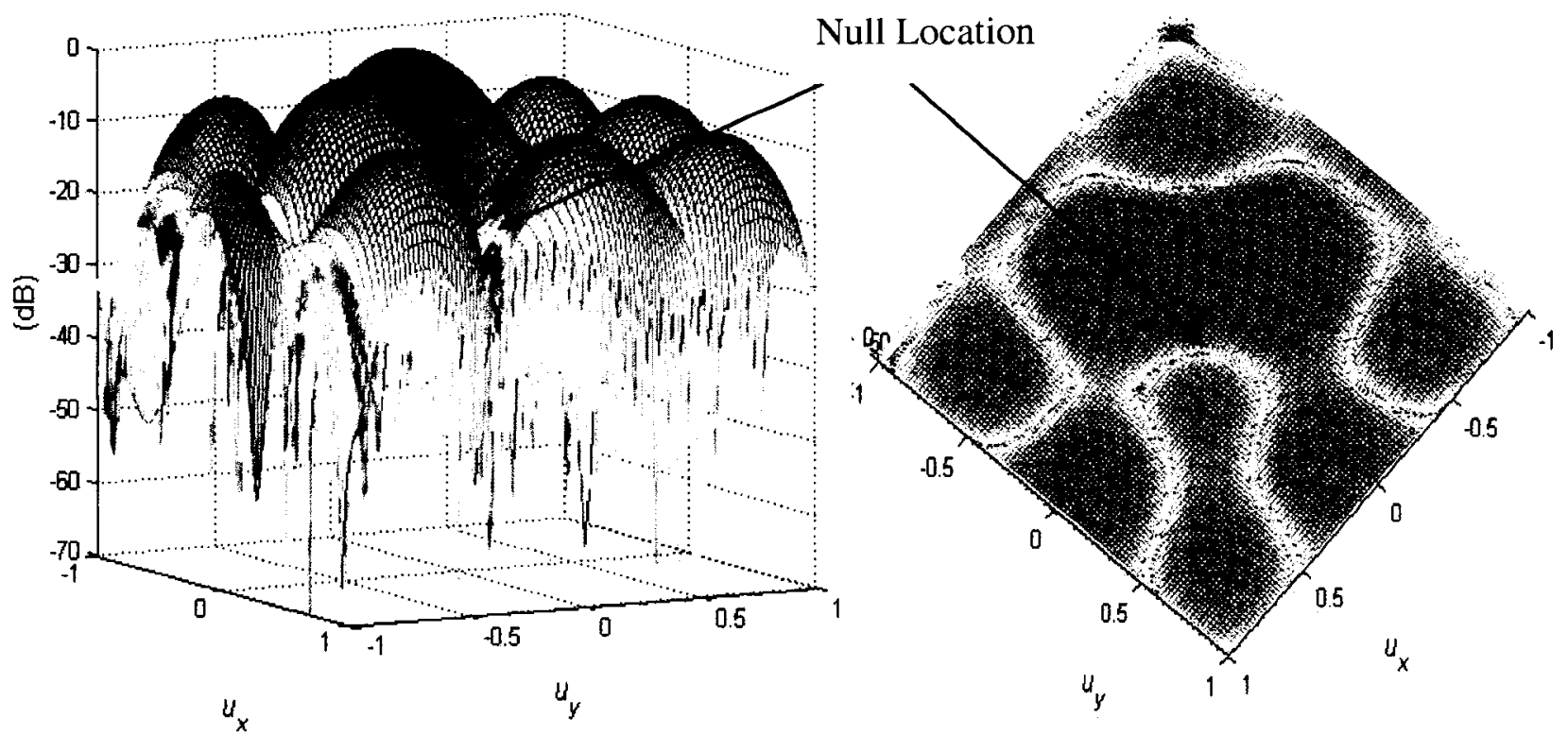

Figure 4.35: Different Views of $4 \times 4$ Array's Beam Pattern with null at

$$
\theta=5^{\circ}, \phi=40^{\circ} \text { or } u_{x}=0.07, u_{y}=0.06
$$


The figures below show the same case but using an $8 \times 8$ standard rectangular array.

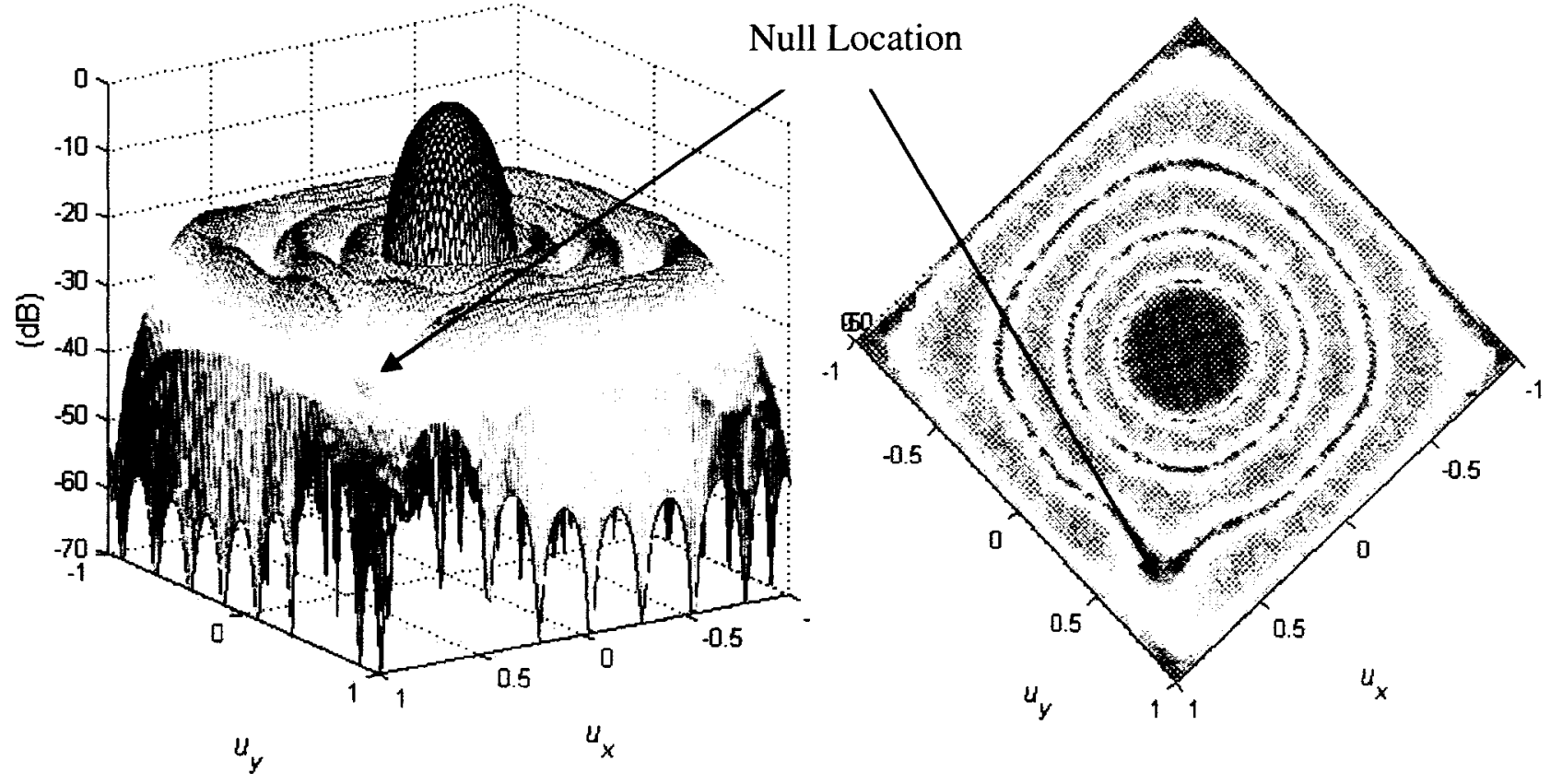

Figure 4.36: Different Views of $8 \times 8$ Array's Beam Pattern with null at $\theta=70^{\circ}, \phi=40^{\circ}$

$$
\text { or } u_{x}=0.72, u_{y}=0.60
$$

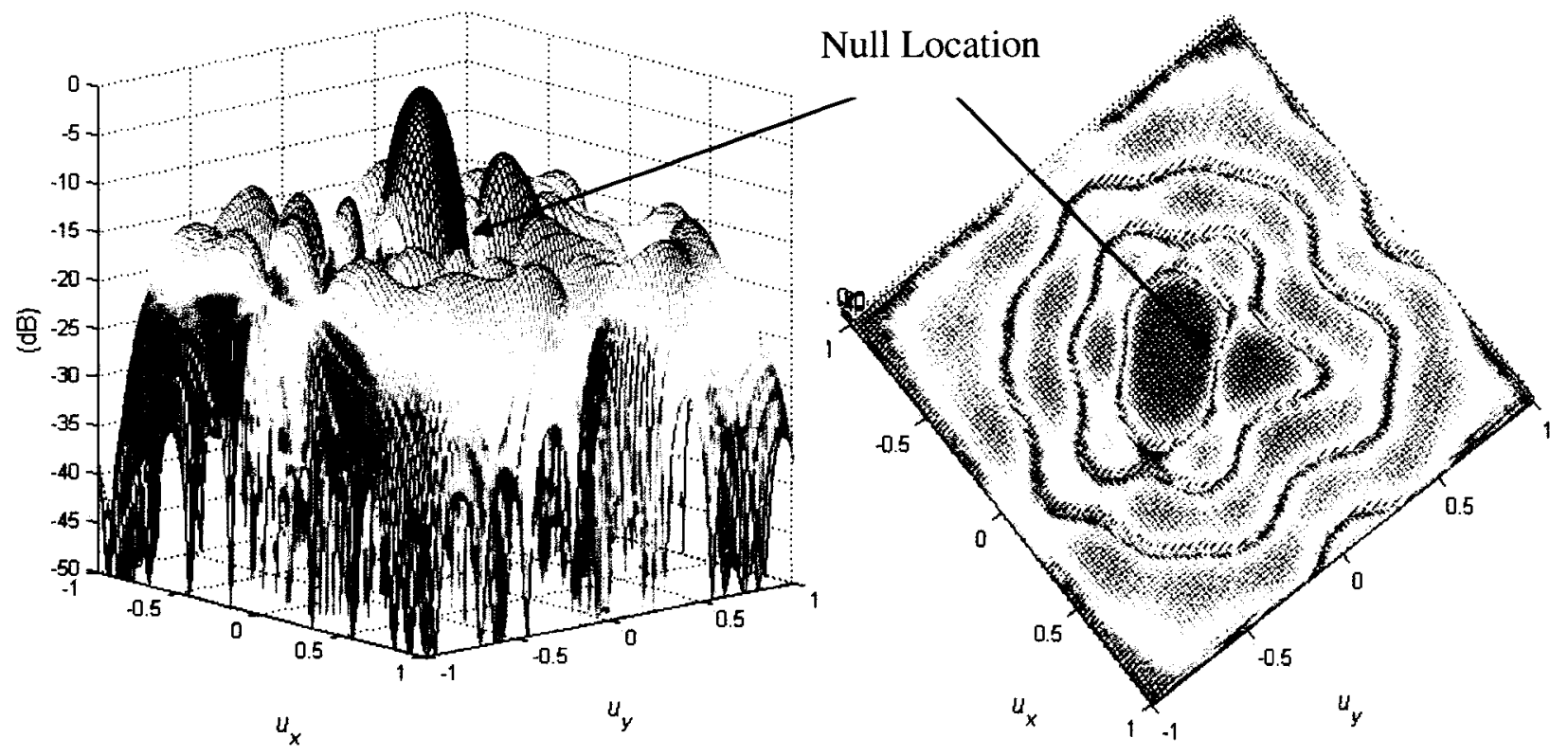

Figure 4.37: Different Views of $8 \times 8$ Array's Beam Pattern with null at $\theta=5^{\circ}, \phi=40^{\circ}$

$$
\text { or } u_{x}=0.07, u_{y}=0.06
$$


The following figures show the optimum beam pattern for a $4 \times 4$ and an $8 \times 8$ standard rectangular array with Dolph-Chebyshev Weighting and with three null placements. The nulls locations are as follow:

$$
\begin{aligned}
& \text { Null 1 @ }\left[\begin{array}{l}
\theta=70^{\circ} \\
\phi=40^{\circ}
\end{array}\right] \text { or }\left[\begin{array}{l}
u_{x}=0.72 \\
u_{y}=0.60
\end{array}\right] \\
& \text { Null 2 @ }\left[\begin{array}{c}
\theta=45^{\circ} \\
\phi=-30^{\circ}
\end{array}\right] \text { or }\left[\begin{array}{l}
u_{x}=0.61 \\
u_{y}=-0.35
\end{array}\right] \\
& \text { Null 3 }\left[\begin{array}{c}
\theta=-80^{\circ} \\
\phi=45^{\circ}
\end{array}\right] \text { or }\left[\begin{array}{l}
u_{x}=-0.70 \\
u_{y}=-0.70
\end{array}\right]
\end{aligned}
$$

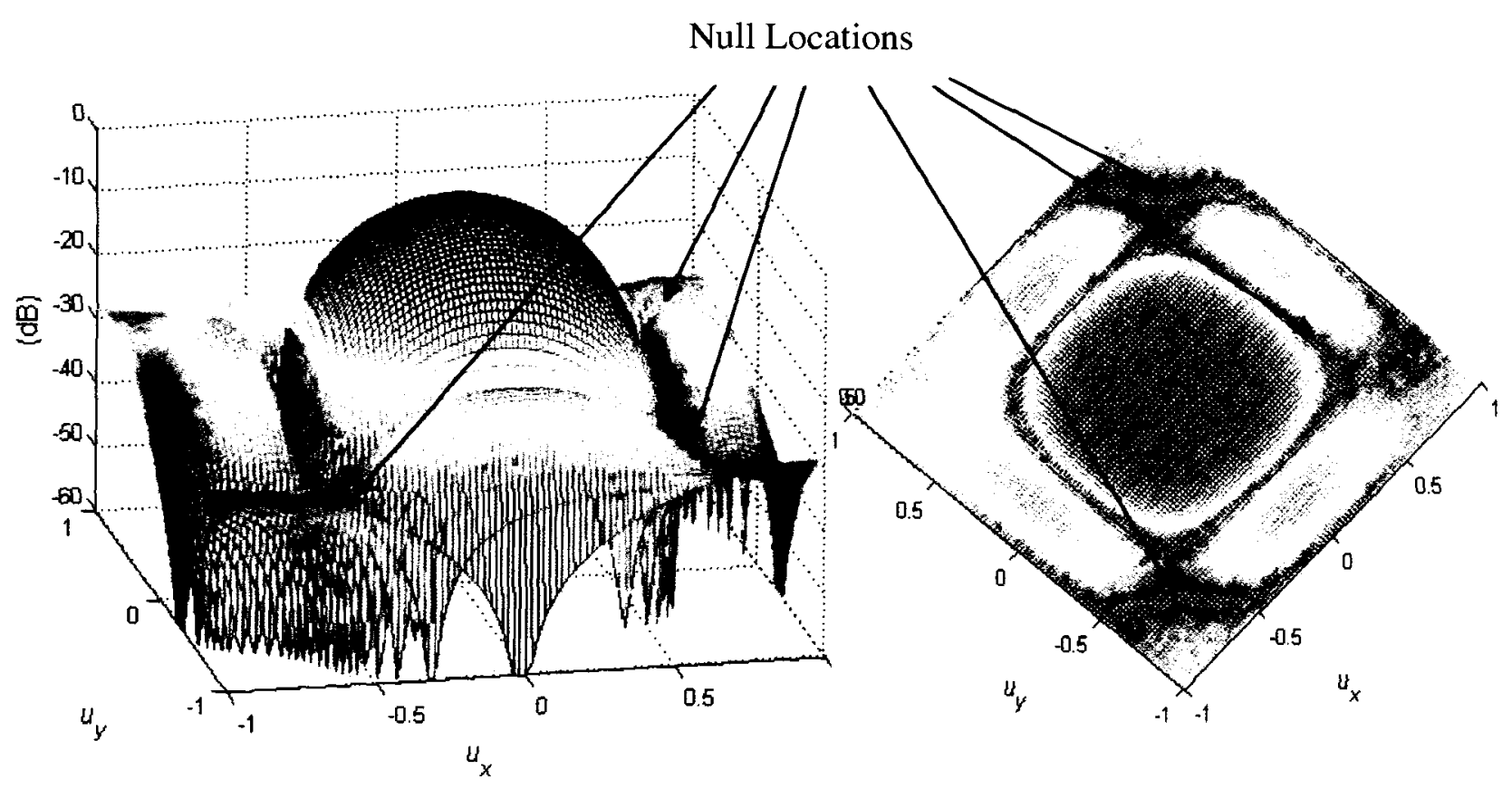

Figure 4.38: $4 \times 4$ Array Optimum Beam Pattern with Three Nulls 


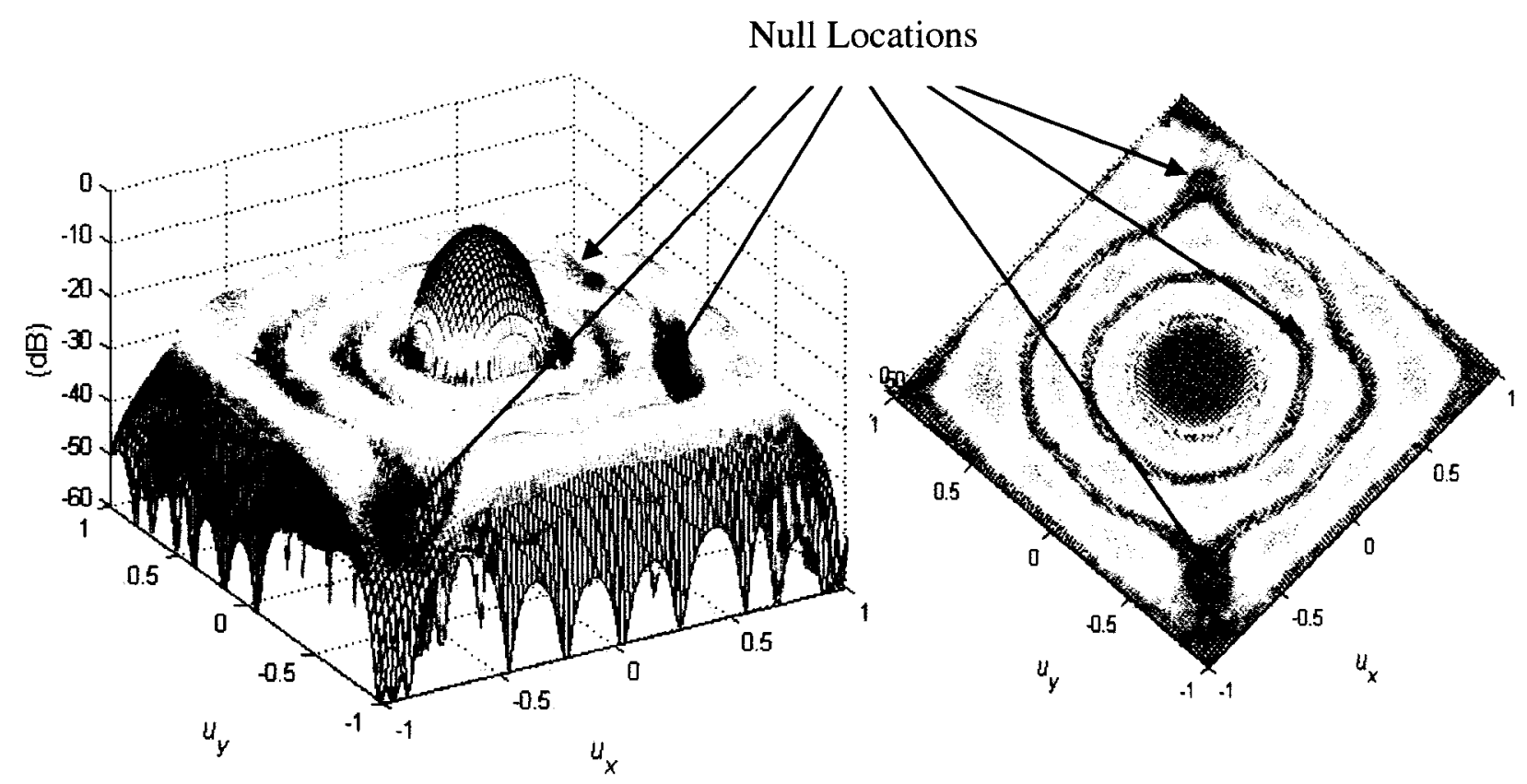

Figure 4.39: $8 \times 8$ Array Optimum Beam Pattern with Three Nulls

\subsubsection{Beam Steering Using Planar Array}

The beam steering (steering the mainlobe electronically) can be implemented for planar arrays too. It is obtained by the extension of the algorithm described in 4.2.2 for a linear array. Using rectangular arrays the mainlobe can be steered in both $\theta$ and $\varphi$. The algorithm was implemented in MATLAB and the following figures show the steering of the mainlobe to $\theta=20^{\circ}$ and $\phi=40^{\circ}$ or $u_{x}=0.26$ and $u_{y}=0.22$ using $4 \times 4$ and $8 \times 8$ rectangular array with Dolph-Chebyshev weighting. 


\section{Steered Mainlobe Location}
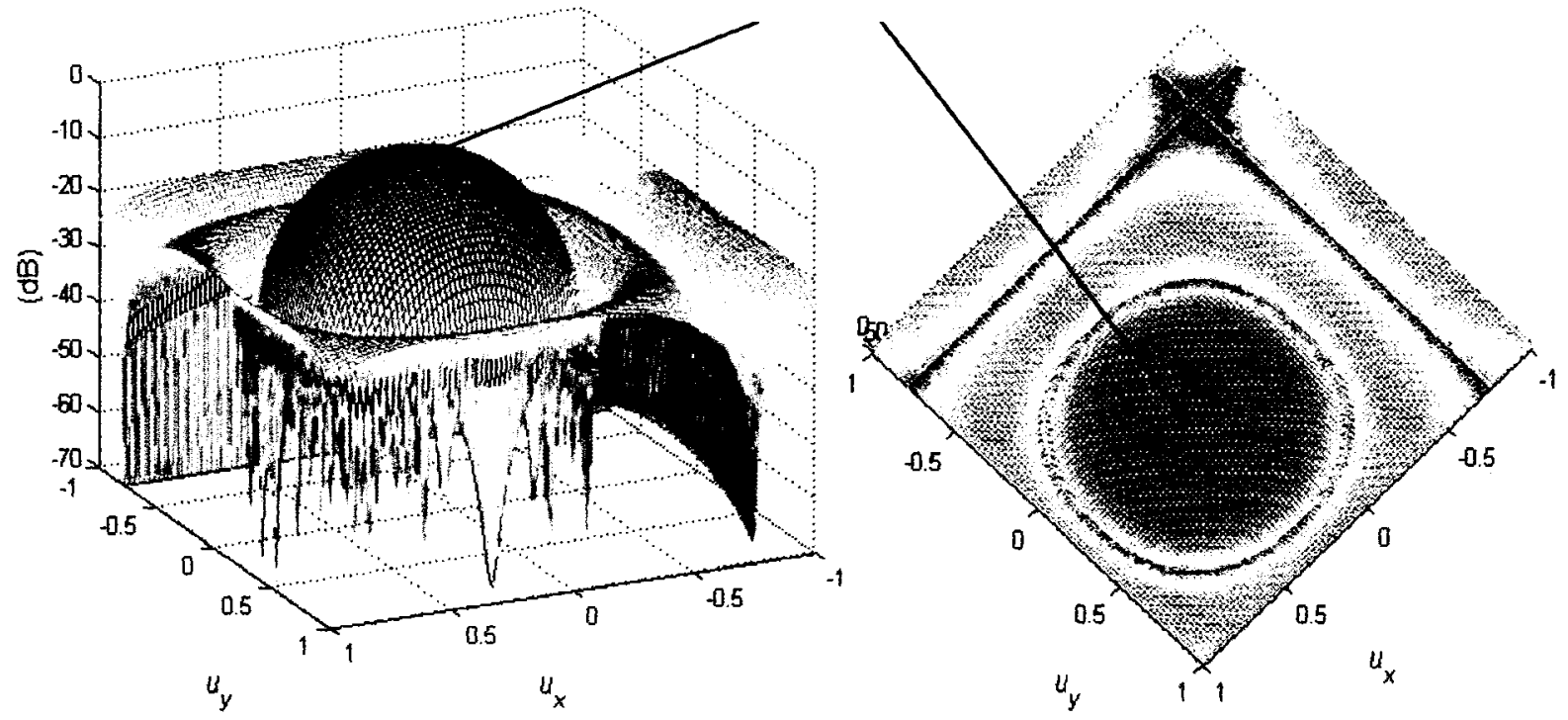

Figure 4.40: Beam Steered to $u_{x}=0.26$ and $u_{y}=0.22$ Using $4 \times 4$ Array

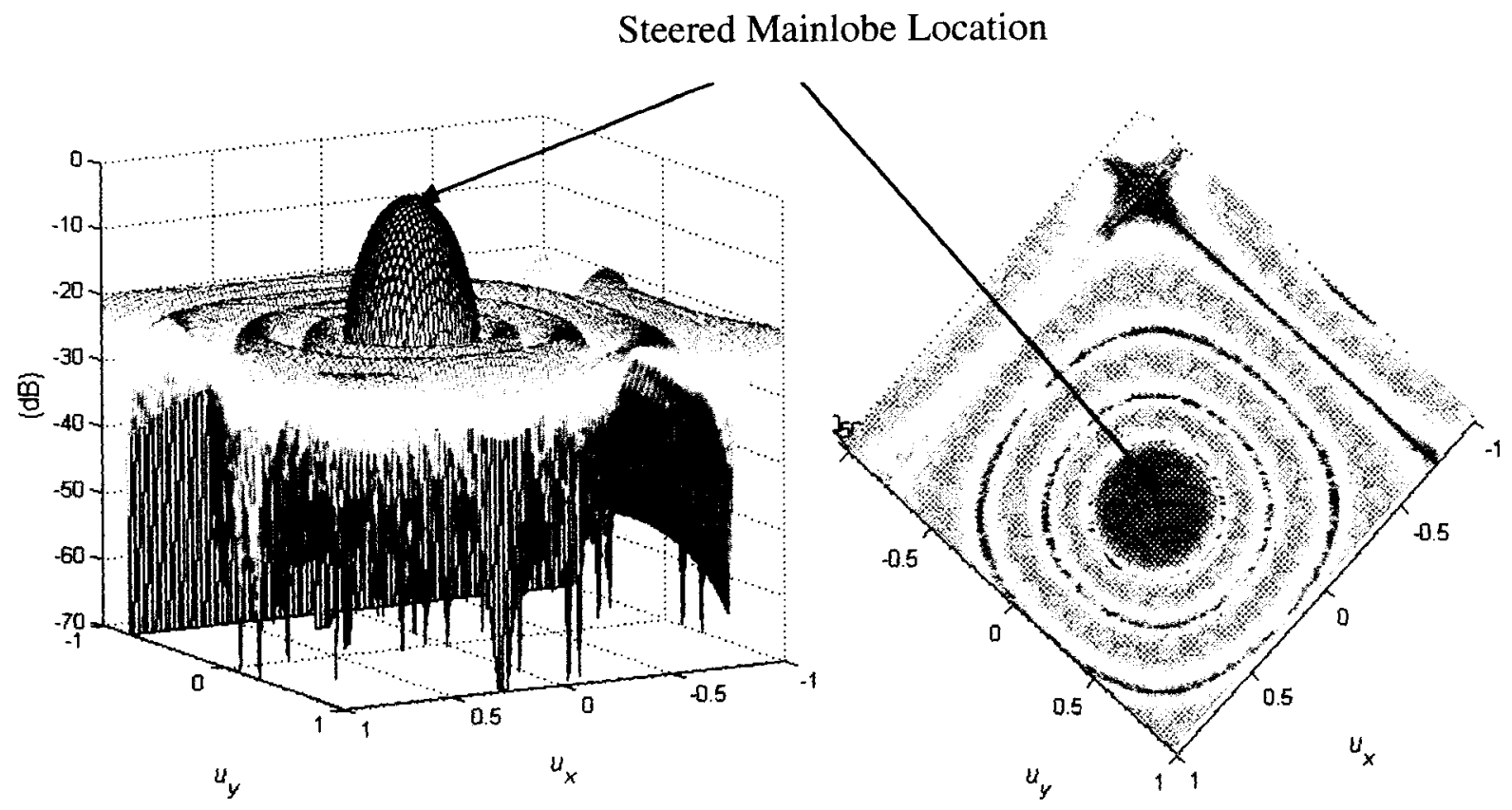

Figure 4.41: Beam Steered to $u_{x}=0.26$ and $u_{y}=0.22$ Using $8 \times 8$ Array 


\subsubsection{Beam+Null Steering Using Planar Array}

Now the two algorithms developed in 4.3 .3 and 4.3 .4 can be combined to achieve the beam+null steering. This is done by first steering the mainlobe to the desired direction and then imposing the null at the direction of jamming/interference signal. It can also steer nulls to multiple jamming/interference directions (depending on the number of elements in the array). The combined algorithm was again implemented in MATLAB and the results are shown in the following figures.

In this example the mainlobe is steered to $\theta=20^{\circ}$ and $\phi=40^{\circ}$ or $u_{x}=0.26$ and $u_{y}=0.22$ (as the previous example) and the null is steered at $\theta=80^{\circ}$ and $\phi=-30^{\circ}$ or $u_{x}=0.85$ and $u_{y}=-0.49$.

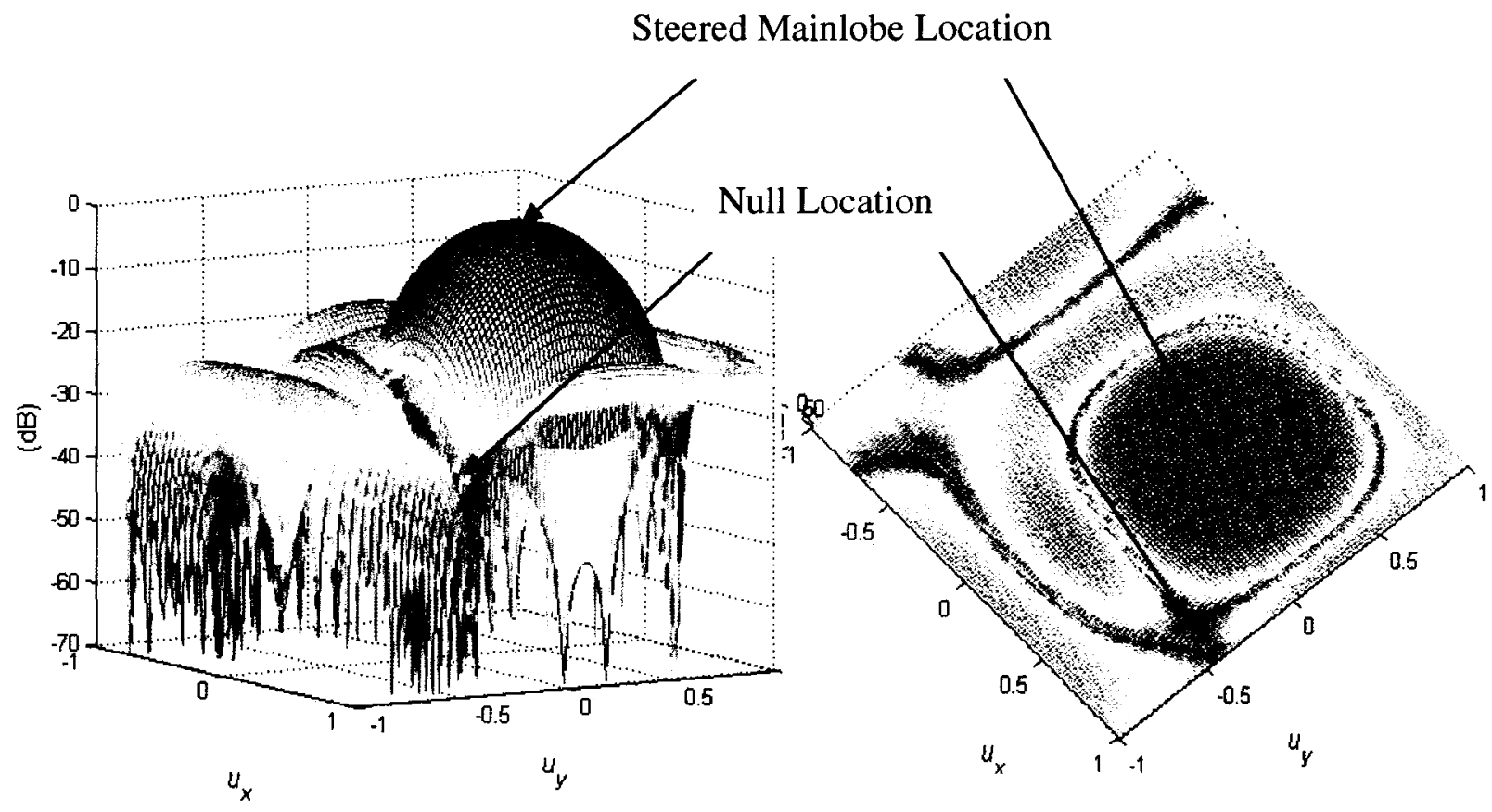

Figure 4.42: $4 \times 4$ Array with Mainlobe Steered to $u_{x}=0.26, u_{y}=0.22$ and Null Steered to $u_{x}=0.85, u_{y}=-0.49$. 




Figure 4.43: $8 \times 8$ Array with Mainlobe Steered to $u_{x}=0.26, u_{y}=0.22$ and Null Steered to $u_{x}=0.85, u_{y}=-0.49$.

\subsection{Minimum Variance Distortionless Response Beamformer}

\subsubsection{MVDR Beamformer Overview}

Consider developing an optimum beamformer for plane-wave signals in the presence of noise, with the objective of estimating the signal waveform. In this section the assumption is that the noise is a sample function of a random process and the signal is an unknown nonrandom plane-wave signal arriving from a known direction. We consider the derivation of an optimum linear array processor, which provides the minimum variance estimate of the incoming signal. In this case, the array processor works as a 
distortionless filter that passes any signal coming from a specified direction undistorted and minimizes the output variance. This processor is called the Minimum Variance Distortionless Response or (MVDR) beamformer also called Capon beamformer [23], [24], [25].

The signal in the frequency-domain can be defined as

$$
\vec{X}(\omega)=\vec{X}_{\text {signal }}(\omega)+\vec{X}_{\text {noise }}(\omega),
$$

The signal vector is

$$
\vec{X}_{\text {signal }}(\omega)=X_{\text {source }}(\omega) \vec{v}\left(\vec{k}_{\text {source }}\right),
$$

where $X_{\text {source }}(\omega)$ is the source signal in frequency-domain and $\vec{v}\left(\vec{k}_{\text {source }}\right)$ is the manifold vector for a signal with wavenumber $\vec{k}_{\text {source }}$.

The zero-mean random noise vector $\vec{X}_{\text {noise }}(\omega)$ has the following spectral matrix, which contains a spatial white noise component:

$$
\vec{S}_{n}(\omega)=\vec{S}(\omega)+\sigma_{\omega}^{2} \vec{I},
$$

where $\sigma_{\omega}^{2}$ is the spectral height of white noise component at each sensor.

The signal snapshot $\vec{X}_{\text {signal }}(\omega)$ can be processed with a $1 \times N$ matrix operation $\vec{W}^{H}(\omega)$ to get the output $\vec{Y}_{\text {signal }}(\omega)$. The distortionless criterion requires the following in the absence of noise: 


$$
\vec{Y}_{\text {signal }}(\omega)=X_{\text {source }}(\omega) \text {, }
$$

In other words

$$
\vec{W}^{H}(\omega) \vec{v}\left(\vec{k}_{\text {source }}\right)=1,
$$

Having this constraint the goal is to minimize the variance of $\vec{Y}_{\text {signal }}(\omega)$ in presence of noise. If $\vec{Y}_{\text {signal }}(\omega)$ is written as

$$
\vec{Y}_{\text {signal }}(\omega)=\vec{X}_{\text {source }}(\omega)+\vec{Y}_{\text {noise }}(\omega)
$$

where the goal is to minimize the expectancy of mean square of the output noise $\left|\vec{Y}_{\text {noise }}(\omega)\right|^{2}$, then

$$
E\left[\left|Y_{\text {noise }}(\omega)\right|^{2}\right]=\vec{W}^{H}(\omega) \vec{S}_{\text {noise }}(\omega) \vec{W}(\omega)
$$

After solving the minimization problem and considering the imposed constraint, we obtain the following for the optimum beamformer:

$$
\vec{W}_{o}^{H}(\omega)=\frac{\vec{v}^{H}\left(\vec{k}_{\text {source }}\right) \vec{S}_{n}^{-1}(\omega)}{\vec{v}^{H}\left(\vec{k}_{\text {source }}\right) \vec{S}_{n}^{-1}(\omega) \vec{v}\left(\vec{k}_{\text {source }}\right)} .
$$

The solution in (4.87) for the optimum beamformer can be seen as a processor illustrated below:

$$
\underset{\vec{X}_{\text {signal }}(\omega)}{\longrightarrow} \frac{\vec{v}^{H}\left(\vec{k}_{\text {source }}\right) \vec{S}_{n}^{-1}(\omega)}{\vec{v}^{H}\left(\vec{k}_{\text {source }}\right) \vec{S}_{n}^{-1}(\omega) \vec{v}\left(\vec{k}_{\text {source }}\right)} \quad \vec{Y}_{\text {signal }}(\omega)
$$

Figure 4.44: MVDR Beamformer 


\subsection{2: MVDR Performance in Presence of Interference}

We now consider a noise process with a single plane-wave interfering signal with array manifold $\vec{v}\left(\vec{k}_{\mathrm{int}}\right)$ in addition to a white noise component (as mentioned above) at each element. This can be written as

$$
\vec{S}_{n}=\sigma_{\omega}^{2} \vec{I}+\Lambda(\omega) \vec{v}\left(\vec{k}_{\mathrm{int}}\right) \vec{v}^{H}\left(\vec{k}_{\mathrm{int}}\right),
$$

where $\Lambda(\omega)$ is the spectrum of the interfering signal and $\vec{I}$ is the identity matrix. For simplicity $\omega$ and $\vec{k}_{\mathrm{int}}$ will be suppressed from here after.

This means the noise spectrum at each element is

$$
S_{n}=\sigma_{\omega}^{2}+\Lambda \vec{v}_{\mathrm{int}} \vec{v}_{\mathrm{int}}^{H}
$$

Using the Woodbury Matrix Identity, that is defined as

$$
(A+U C V)^{-1}=A^{-1}-A^{-1} U\left(C^{-1}+V A^{-1} U\right)^{-1} V A^{-1} .
$$

$\vec{S}_{n}^{-1}(\omega)$ is found to be:

$$
\vec{S}_{n}^{-1}=\frac{1}{\sigma_{\omega}^{2}}\left[\vec{I}-\frac{\Lambda \vec{v}_{\mathrm{int}} \vec{v}_{\mathrm{int}}^{H}}{\sigma_{\omega}^{2}+N \Lambda}\right]
$$

By substituting (4.91) in (4.87), the optimum beamformer in the presence of interference can be achieved:

$$
\vec{W}_{o}^{H}=\frac{N}{\Phi \sigma_{\omega}^{2}}\left(\frac{\vec{v}_{\text {source }}^{H}}{N}-\rho_{\text {source_int }} \frac{\Lambda}{\sigma_{\omega}^{2}+N \Lambda} \cdot \vec{v}_{\mathrm{int}}^{H}\right),
$$


where

$$
\Phi=\frac{1}{\sigma_{\omega}^{2}} N\left(1-\left|\rho_{\text {source_int }}\right|^{2} \frac{N \Lambda}{\sigma_{\omega}^{2}+N \Lambda}\right)
$$

and $\rho_{\text {source_int }}$ is the spatial correlation coefficient between the source (desired) signal and the interference signal and is defined as

$$
\rho_{\text {source }- \text { int }}=\frac{1}{N} \vec{v}_{\text {source }}^{H} \vec{v}_{\mathrm{int}} .
$$

The optimum beam pattern can be found by substituting (4.92) in (4.20):

$$
B_{o}\left(\vec{k}_{\text {signal }}\right)=\frac{N}{\Phi \sigma_{\omega}^{2}}\left(B\left(\vec{k}_{\text {source }}\right)-\left(\frac{N \rho_{\text {source_int }} \sigma_{\text {int }}^{2}}{N \sigma_{\text {int }}^{2}+1}\right) B\left(\vec{k}_{\mathrm{int}}\right)\right),
$$

where $\sigma_{\mathrm{int}}^{2}$ is the interference to white noise ratio (INR).

$B\left(\vec{k}_{\text {source }}\right)$ is the conventional array beam pattern (4.17) in the direction of $\vec{k}_{\text {source }}$ :

$$
B\left(\vec{k}_{\text {source }}\right)=\frac{1}{N} \vec{v}^{H}\left(\vec{k}_{\text {source }}\right) \vec{v}(\vec{k}),
$$

and $B\left(\vec{k}_{\mathrm{int}}\right)$ is the conventional array beam pattern in the direction of interference $\vec{k}_{\mathrm{int}}$ :

$$
B\left(\vec{k}_{\mathrm{int}}\right)=\frac{1}{N} \vec{v}^{H}\left(\vec{k}_{\mathrm{int}}\right) \vec{v}(\vec{k})
$$

The optimum beam pattern defined in (4.95) can be seen as the beam pattern in the direction of the desired signal minus a constant times the beam pattern in the direction of interference. This beamformer is illustrated on the next page in Figure 4.45. 


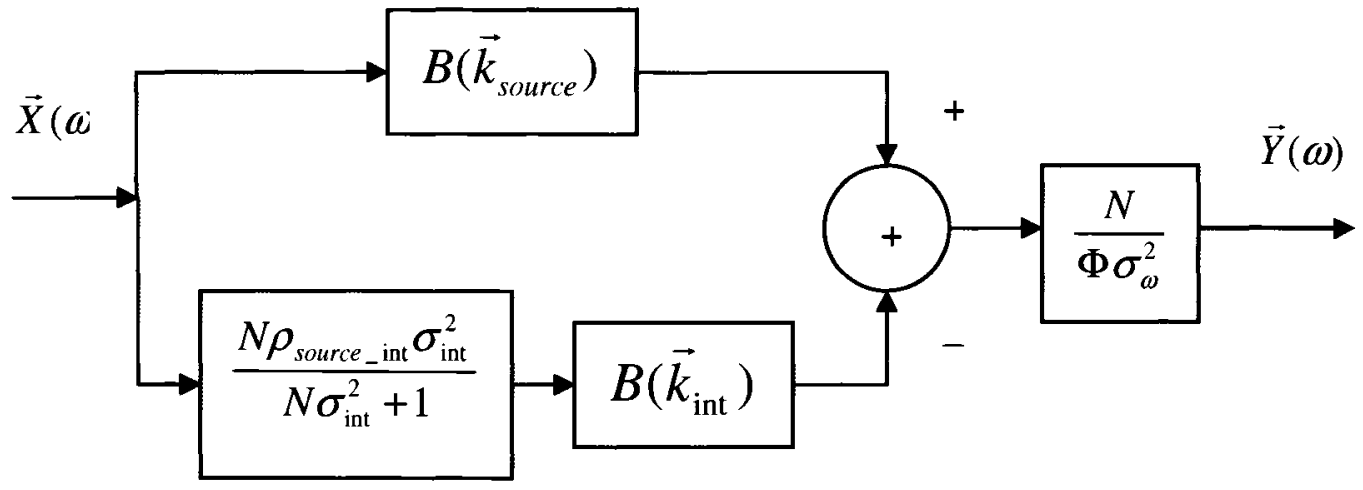

Figure 4.45: MVDR Beamformer in Presence of Interference

The figure below shows the linear array's optimum beam pattern using the MVDR beamformer in presence of interference. The interference-to-Noise ratio (INR) is assumed to be $10 \mathrm{~dB}$. The interferences are located at $u=[0.2,0.866]$ or $\theta=\left[78.5^{\circ}, 30^{\circ}\right]$. As it can be seen when the interference location is located on the mainlobe, the imposed null causes the increase in the sidelobe levels. The same can be seen for the case when the null is suppressing a sidelobe, which results in the increase in the level of neighboring sidelobes.
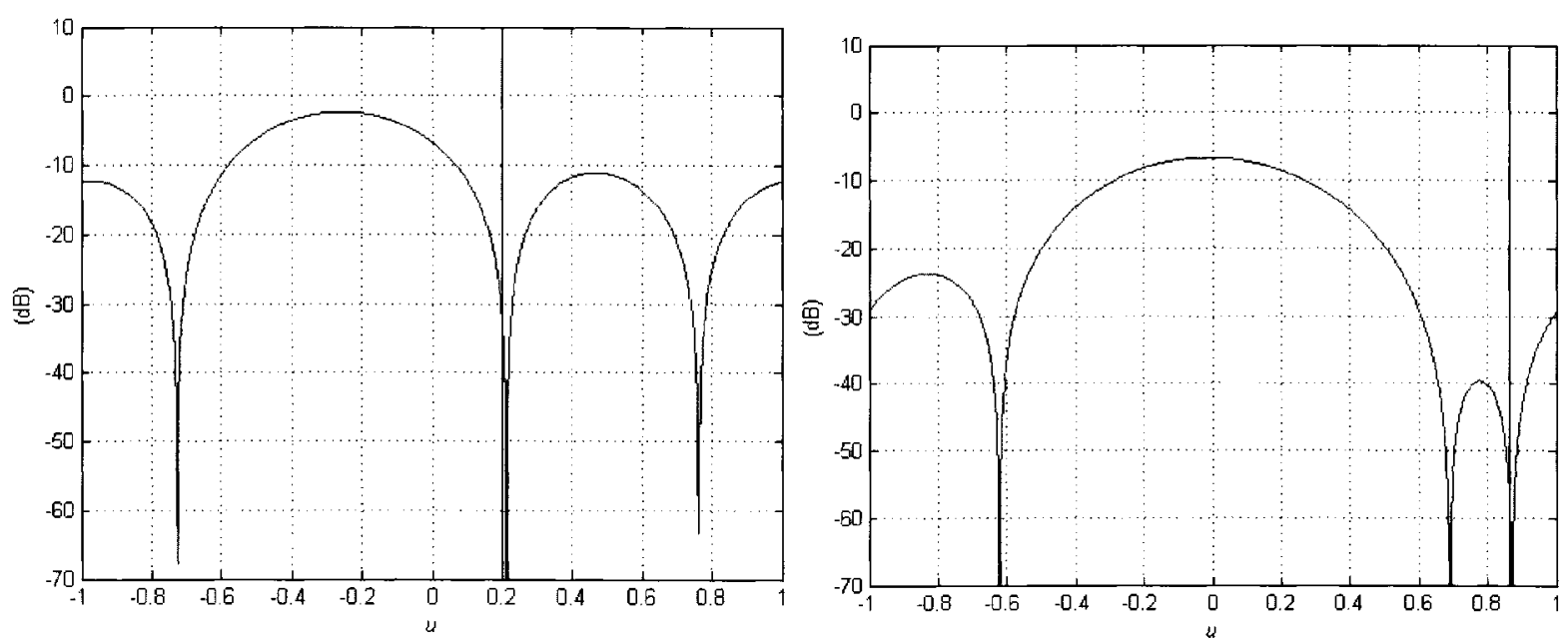

Figure 4.46: 4-Element Array Pattern in Presence of Interference 

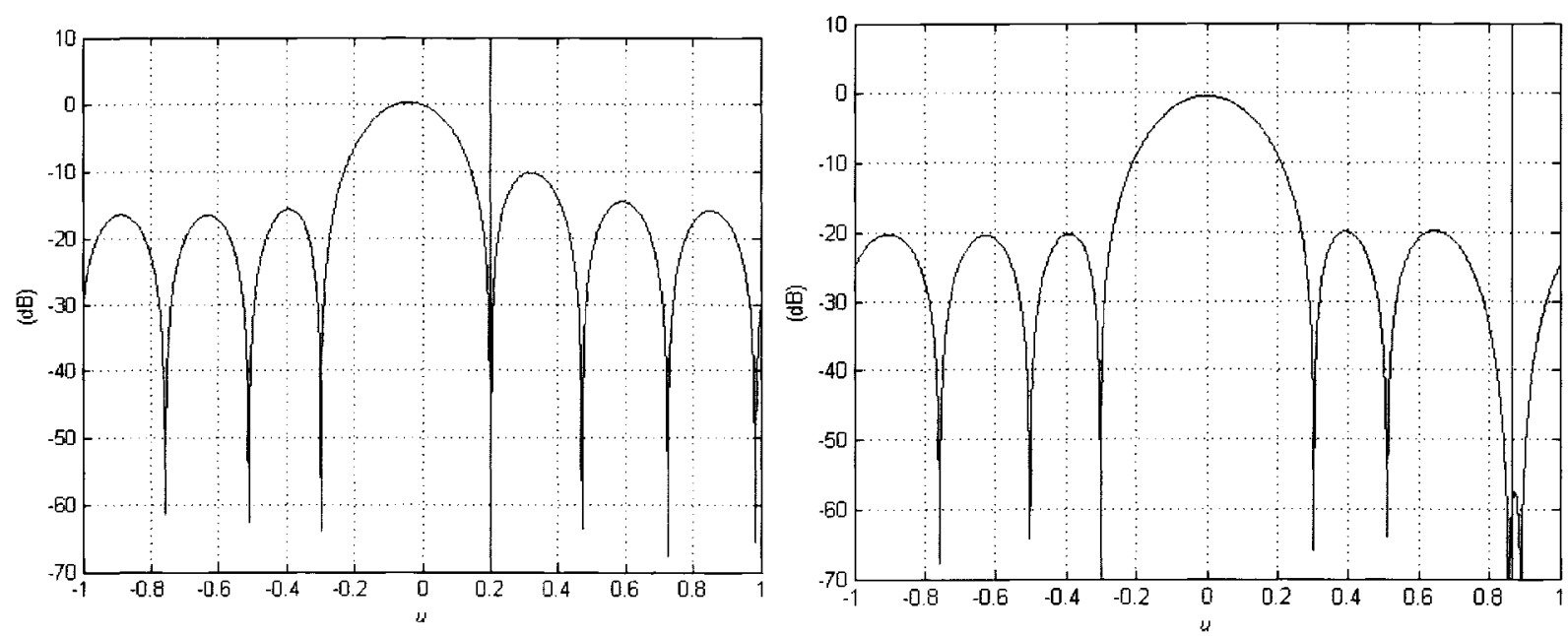

Figure 4.47: 8-Element Array Pattern in Presence of Interference

As it was done for the null steering in section 4.3.3, the MVDR beamformer can be implemented for planar arrays. Using MATLAB the following beam patterns were plotted for an interference with INR of $10 \mathrm{~dB}$. The Interference is located at $u=[0.47,0.81]$ or $\theta=70^{\circ}, \phi=60^{\circ}$.

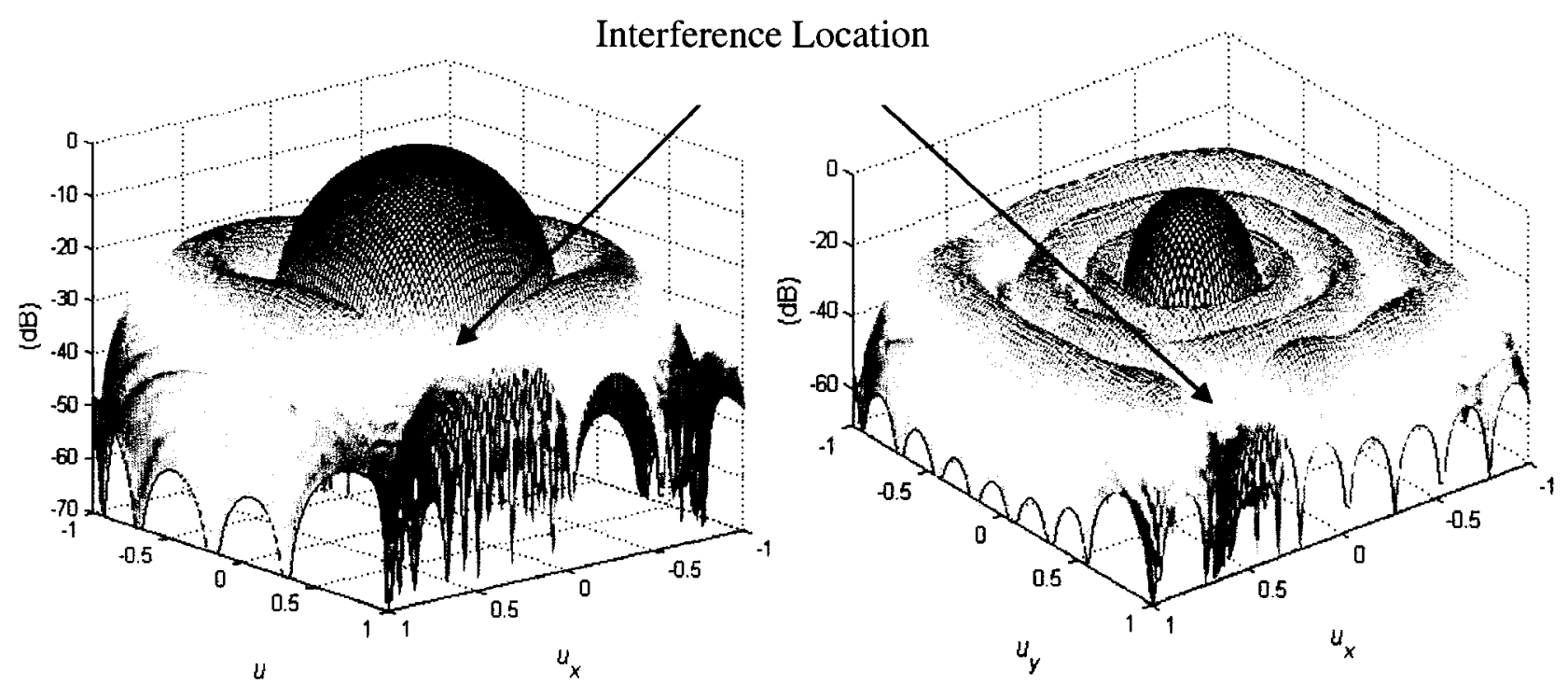

Figure 4.48: $4 \times 4$ and $8 \times 8$ Array Beam Pattern in Presence of Interference 


\subsection{Conclusion}

This chapter started with an overview of the array processing and beam forming concepts. Then the optimum beam pattern for linear arrays was obtained using the DolphChebyshev weighting. Null steering and beam steering in the case of linear arrays were discussed afterwards. This was followed by the case for planar arrays and again the null steering and beam steering algorithms were implemented and the results of simulation in MATLAB were presented. In addition to these, the results for beam+null steering were provided in this chapter.

Due to the accuracy and resolution issues the minimum number of array elements was chosen to be 4 , and all the results were presented for both 4 and 8 -element arrays ( $4 \times 4$ and $8 \times 8$ in case of planar arrays). The results can be compared to see the effect of the increase in the number of elements on the array performance. 


\section{Chapter 5: Concluding Remarks}

This chapter briefly summarizes the research covered in this thesis and suggests some direction for future research in this field.

\subsection{Summary of the Research}

This thesis consists of two major parts. The first part gives a brief overview on the GPS technology and its history in addition to a summary of the interference and jamming scenarios and the anti-jamming techniques. The second part of the thesis contains the major research work done by the author and covers the concept of optimum array processing in detail. It shows the result of algorithms developed for null- and beamsteering.

The chapter on the interference and anti-jamming techniques presents a summary of the techniques, which can be as simple as analog front end filtering, or as complex as adaptive algorithms, developed in the digital domain. As was discussed earlier, when the techniques get more complex it results in very large and expensive units. This limits their use in many scenarios, where a single beamformer with null steering capability can be much more practical.

The optimum beamformer was developed for both linear and planar arrays and the results were presented including the case for multiple null placements. Although the algorithms for planar arrays are much more complicated, they have the ability to null the 
interference signal in both azimuth and elevation angles. The Dolph-Chebyshev weighting was considered to achieve the optimum beam pattern, since it can provide the optimum mainlobe beam width for a selected sidelobe level.

All the results obtained from MATLAB are presented for both 4 and 8 element arrays ( $4 \times 4$ and $8 \times 8$ in case of planar arrays), which can be used to compare the resolution and accuracy of the nulling performance. It shows clearly that, as the number of the elements increases deeper and sharper nulls can be achieved, which helps prevent the elimination of desired GPS signal. This is the reason for selecting the minimum number of array elements to be 4 ( $4 \times 4$ in case of planar arrays).

\subsection{Possible Future Work}

Throughout this report the angle of arrival of jamming signal was assumed to be known and arbitrary directions were used in the simulations. In a continuation of this work, as an extensive research subject, an interesting problem to look into would be the angle of arrival estimation (in the digital domain). Combining the present research with the AoA estimator would create an adaptive null steering beamformer.

The author collaborated with his co-researcher, Mr. Ehsan Gharibdoust, in design of a tri-band microstrip antenna for GPS application. The antenna size was minimized to make it suitable for implementation in a rectangular array. This antenna array could be implemented in future work to test the performance of the optimum algorithms discussed in this thesis. 


\section{References}

[1] J. V. Carrol, K. Van Dyke, and J. H. Kraemer, "Vulnerability Assessment of the U.S. Transportation Infrastructure that Relies on GPS”, ION GPS 2001, Salt Lake City, Utah, September 2001

[2] E. D. Kaplan, "Understanding GPS: Principles and Applications”, Artech House Publishers, Boston, 1996

[3] J. P. Stenbit, “GPS Standard Positioning Service Performance”, Assistant Secretary of Defense, October 2001

[4] P. H. Dana, "Global Positioning System Overview”, University of Colorado at Boulder, 2000

[5] J. F. Raquet, "Short Course: GPS 101", Joint Navigational Conference, Las Vegas, NV, April 2003

[6] I. M. Weiss, A. W. Morrison, "GPS Anti-Jam: Picking the Right Technology for Interference Suppression", Joint Navigational Conference, Las Vegas, NV, April 2003

[7] H. F. Hegels, "The Effects of RFI/Jamming on the Current Generation NAVSTAR GPS”, RTO SET Symposium, Netherlands, October 2002

[8] S. G. Carlson, C. A. Popeck, M. H. Stockmaster, C. E. McDowell, “Rockwell Collins' Flexible Digital Anti-Jam Architecture”, ION GPS/GNSS Conference, Portland, OR, September 2003 
[9] M. Trinkle, "The Comparative Analysis of GPS Antenna Anti-Jam Technologies; Part I", Cooperative Research Centre for Sensor Signal and Information Processing, Adelaide University, Australia, November 2001

[10] M. Trinkle, "The Comparative Analysis of GPS Receiver Anti-Jam Technologies; Part 2", Cooperative Research Centre for Sensor Signal and Information Processing, Adelaide University, Australia, September 2001

[11] D. Gray, M. Trinkle, "Overview of GPS Receiver Anti-Jam Technologies", Cooperative Research Centre for Sensor Signal and Information Processing, Adelaide University, Australia, April 2001

[12] D. Gray, M. Trinkle, "Overview of GPS Antenna Anti-Jam Technologies", Cooperative Research Centre for Sensor Signal and Information Processing, Adelaide University, Australia, June 2001

[13] J. Bird, "GPS Navwar", Navwar Conference, Defence Research and Development Canada, Ottawa, November 2004

[14] C. A. Balanis, “Antenna Theory: Analysis and Design”, Wiley, New York, 1982

[15] S. J. Orfanidis, "Electromagnetic Waves and Antennas", Rutgers University, Piscataway, NJ, 2004

[16] H. L. Van Trees, "Detection, Estimation, and Modulation Theory, Part IV", Wiley Interscience, New York, 2002

[17] A. V. Openheim, R. W. Schafer, "Discrete-Time Signal Processing”, Prentice-Hall, Englewood Cliffs, NJ, 1989 
[18] D. H. Johnson, D. E. Dudgeon, "Array Signal Processing", Prentice-Hall, Englewood Cliffs, NJ, 1993

[19] R. J. Mailloux, “Phased Array Antennas”, Artech House, Boston, 1994

[20] L. K. Hong, "A Beamforming Algorithm for Planar Arrays", IEEE Transaction, vol. AE-19, pp. 595-605, July 1983

[21] T. Haynes, "A Primer on Digital Beamforming", Spectrum Signal Processing Journall, March 1998

[22] C. L. Dolph, "A Current Distribution for Broadside Arrays which Optimizes the Relationship between Beamwidth and Sidelobe Level", IRE, vol. 34, pp. 335-348, June 1946

[23] J. Capon, "High-Resolution Frequency-Wavenumber Spectrum Analysis", IEEE, vol.57, pp. 1408-1418, August 1969

[24] M. D. Zoltowsky, "On the Performance of the MVDR Beamformer in the Presence of Interference", IEEE Transaction Acoustic Signal Processing, vol. ASP-36, pp. 940-947, June 1988

[25] P. Sinha, A. D. George, K. Kim, "Algorithms for Robust Broadband MVDR Beamforming", High-Performance Computing and Simulation Laboratory, University of Florida, 2000 


\section{Appendix}

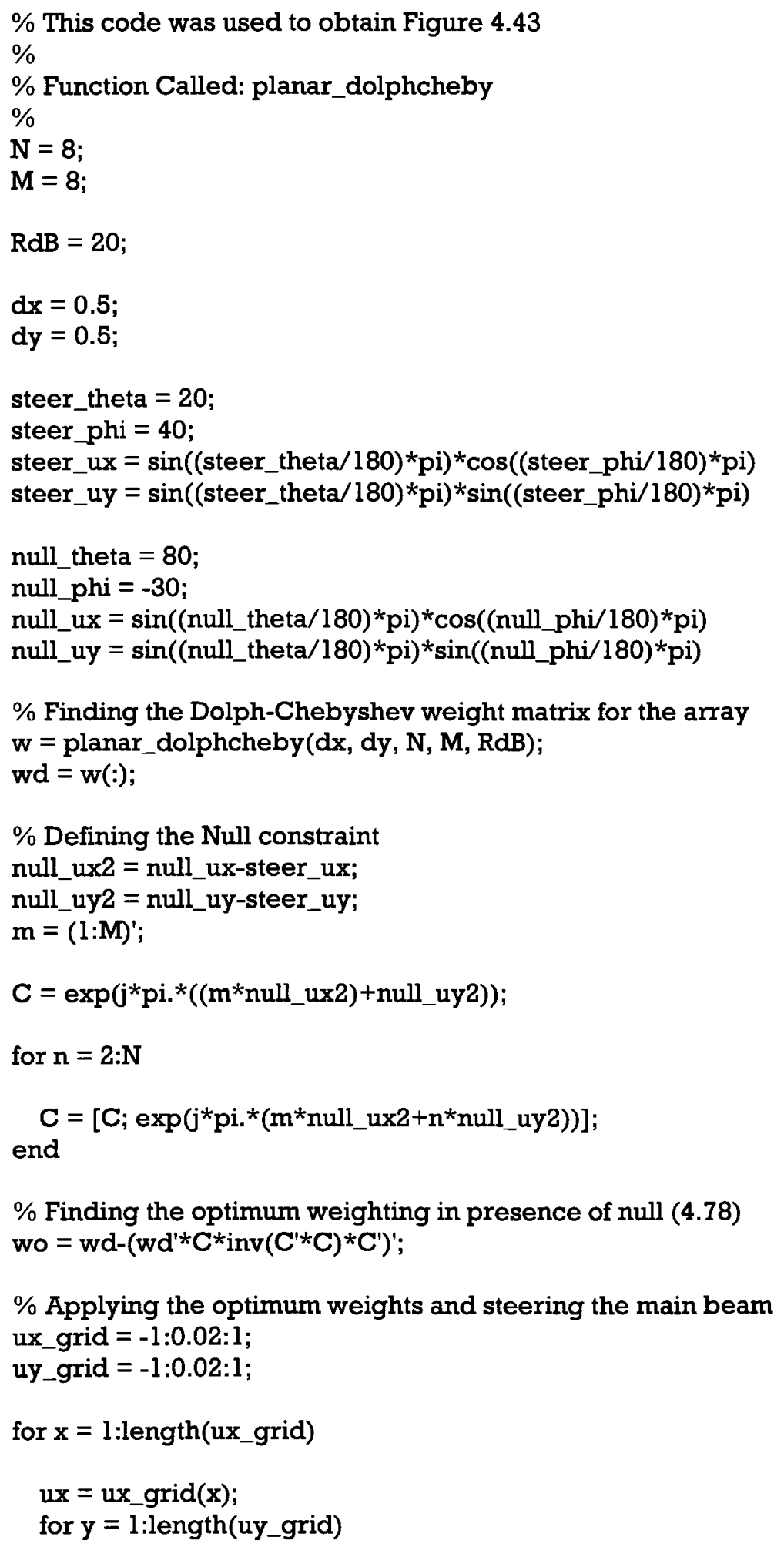




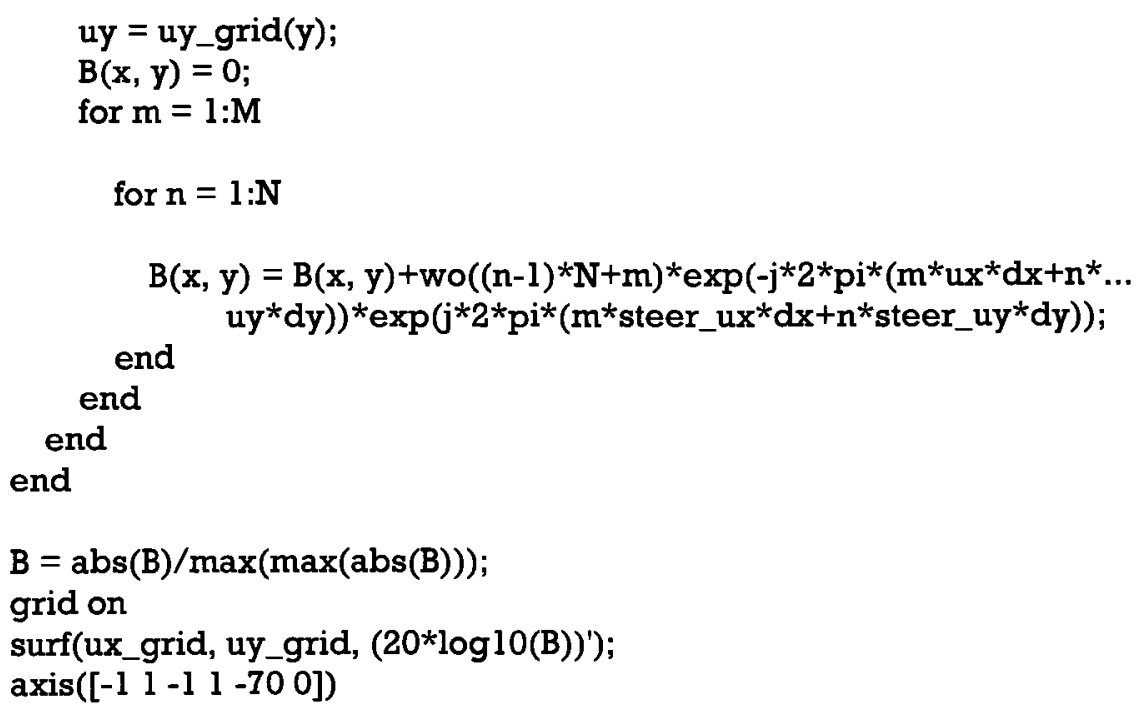

The planar_dolpcheby.m function used in the above code is defined as follow:

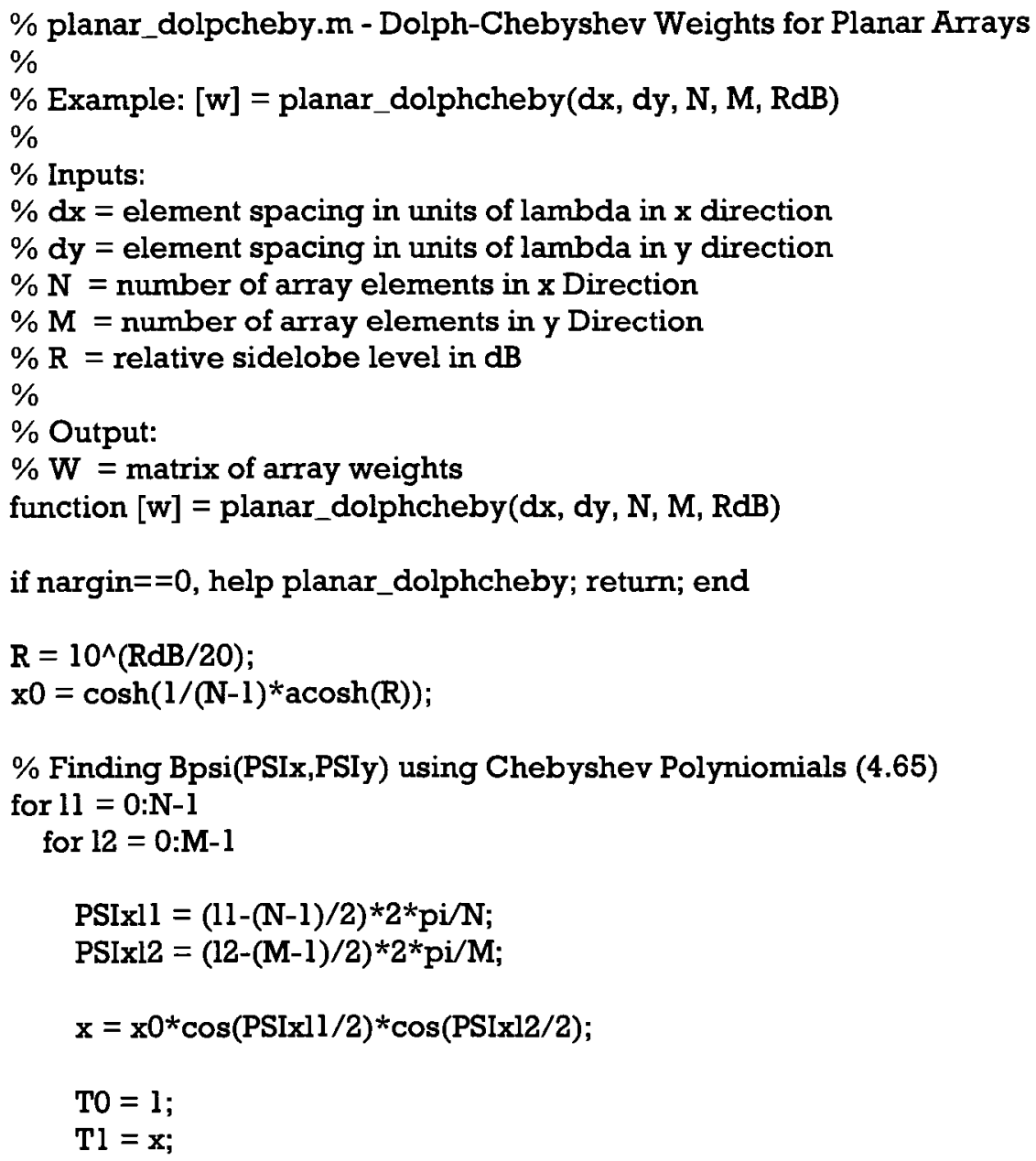




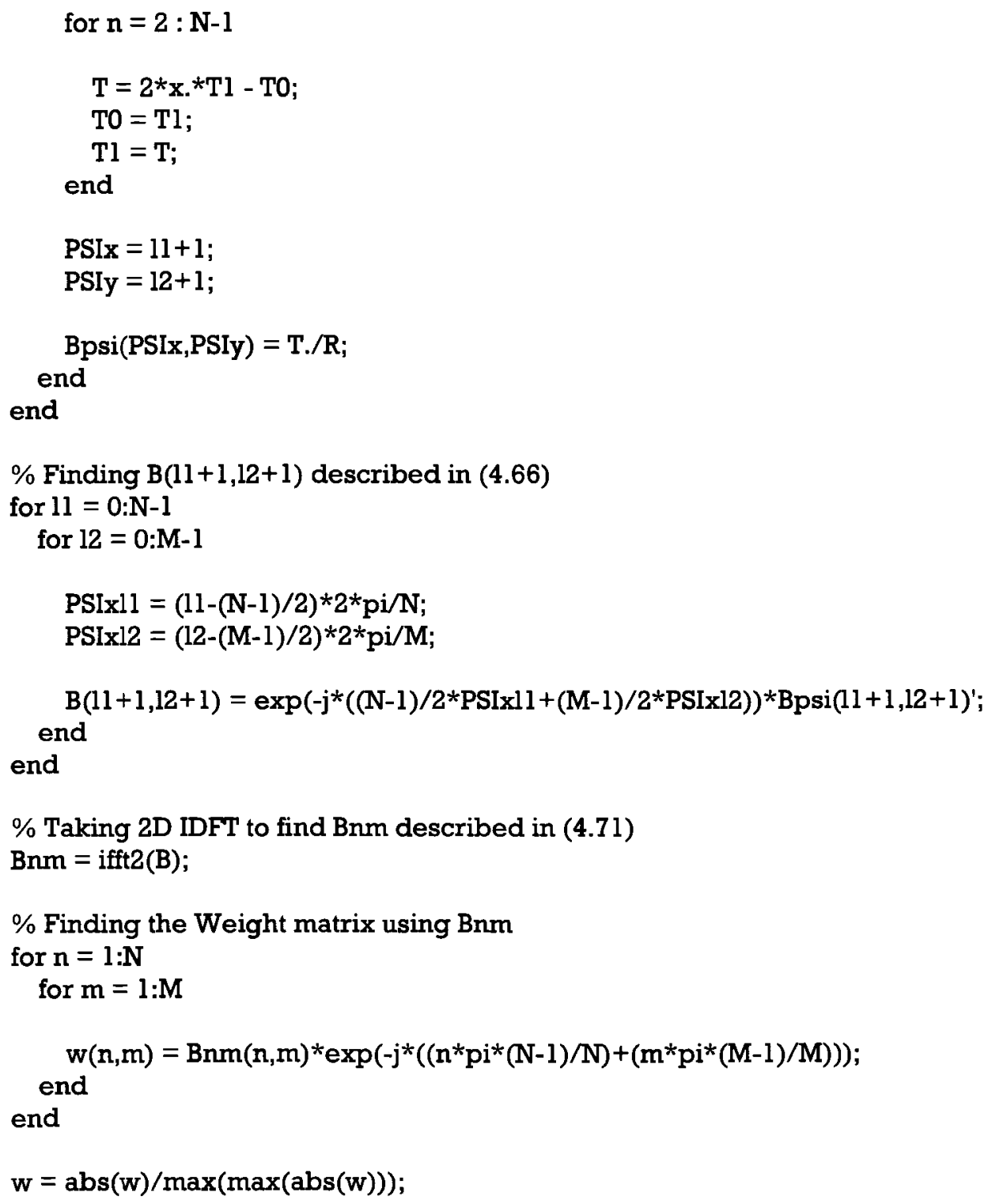

